University of Louisville

ThinkIR: The University of Louisville's Institutional Repository

Electronic Theses and Dissertations

$12-2016$

\title{
A CAD system for early diagnosis of autism using different imaging modalities.
}

Marwa Maher Tawfik Ismail

University of Louisville

Follow this and additional works at: https://ir.library.louisville.edu/etd

Part of the Biomedical Engineering and Bioengineering Commons

\section{Recommended Citation}

Ismail, Marwa Maher Tawfik, "A CAD system for early diagnosis of autism using different imaging modalities." (2016). Electronic Theses and Dissertations. Paper 2599.

https://doi.org/10.18297/etd/2599

This Doctoral Dissertation is brought to you for free and open access by ThinkIR: The University of Louisville's Institutional Repository. It has been accepted for inclusion in Electronic Theses and Dissertations by an authorized administrator of ThinkIR: The University of Louisville's Institutional Repository. This title appears here courtesy of the author, who has retained all other copyrights. For more information, please contact thinkir@louisville.edu. 


\title{
A CAD SYSTEM FOR EARLY DIAGNOSIS OF AUTISM USING DIFFERENT IMAGING MODALITIES
}

\author{
By \\ Marwa Maher Tawfik Ismail \\ M.Sc., Cairo University, Cairo, Egypt, 2008
}

\begin{abstract}
A Dissertation
Submitted to the Faculty of the

J. B. Speed School of the University of Louisville in Partial Fulfillment of the Requirements for the Degree of

Doctor of Philosophy in Electrical Engineering

Department of Electrical and Computer Engineering

University of Louisville

Louisville, Kentucky
\end{abstract}

December, 2016 



\title{
A CAD SYSTEM FOR EARLY DIAGNOSIS OF AUTISM USING DIFFERENT IMAGING MODALITIES
}

\author{
By \\ Marwa Maher Tawfik Ismail \\ M.Sc., Cairo University, Cairo, Egypt, 2008
}

A Dissertation Approved on

November 15th, 2016

by the Following Dissertation Committee:

Ayman El-Baz, Ph.D., Dissertation Director

Tamer Inanc, Ph.D.

Olfa Nasraoui, Ph.D.

Andre Faul, Ph.D. 


\section{ACKNOWLEDGMENTS}

I would like to express my deepest gratitude to my advisor Dr. Ayman El-Baz for his guidance, support, and encouragement to pursue a Ph.D. degree in the medical imaging field.

I would like to acknowledge the other members of my $\mathrm{Ph} . \mathrm{D}$. committee for spending time and effort in reading and reviewing my work Dr. Tamer Inanc, Dr. Olfa Nasraoui, and Dr. Andre Faul.

I would also like to thank Mr. Andrew Switala with whom I enjoyed very fruitful and stimulating research collaborations. Our discussions were always a great pleasure, and led to significant improvements in the content and exposition of this work. He has been of tremendous help to me during this study.

Also, I would like to thank all BioImaging Lab members for their friendship and assistance. I am especially grateful to Ahmed Soliman, Matthew Nitzken, Fahmi Khalifa, Ahmed EINakib, Mohammad Shehata, Omar Dekhil, Ahmed Shalaby, and the rest of the lab members.

Finally, words cannot describe how I am indebted to my husband Omar for all his sacrifices made during the journey of this study. 


\begin{abstract}
A CAD SYSTEM FOR EARLY DIAGNOSIS OF AUTISM USING DIFFERENT

IMAGING MODALITIES
\end{abstract}

Marwa Maher Tawfik Ismail

November 15, 2016

The term "autism spectrum disorder" (ASD) refers to a collection of neuro-developmental disorders that affect linguistic, behavioral, and social skills. Autism has many symptoms, most prominently, social impairment and repetitive behaviors. It is crucial to diagnose autism at an early stage for better assessment and investigation of this complex syndrome. There have been a lot of efforts to diagnose ASD using different techniques, such as imaging modalities, genetic techniques, and behavior reports. Imaging modalities have been extensively exploited for ASD diagnosis, and one of the most successful ones is Magnetic resonance imaging (MRI), where it has shown promise for the early diagnosis of the ASDrelated abnormalities in particular.

Magnetic resonance imaging (MRI) modalities have emerged as powerful means that facilitate non-invasive clinical diagnostics of various diseases and abnormalities since their inception in the 1980s. After the advent in the nineteen eighties, MRI soon became one of the most promising non- invasive modalities for visualization and diagnostics of ASD-related abnormalities. Along with its main advantage of no exposure to radiation, high contrast, and spatial resolution, the recent advances to MRI modalities have notably 
increased diagnostic certainty. Multiple MRI modalities, such as different types of structural MRI (sMRI) that examines anatomical changes, and functional MRI (fMRI) that examines brain activity by monitoring blood flow changes, have been employed to investigate facets of ASD in order to better understand this complex syndrome.

This work aims at developing a new computer-aided diagnostic (CAD) system for autism diagnosis using different imaging modalities. It mainly relies on making use of structural magnetic resonance images for extracting notable shape features from parts of the brain that proved to correlate with ASD from previous neuropathological studies. Shape features from both the cerebral cortex $(\mathrm{Cx})$ and cerebral white matter $(\mathrm{CWM})$ are extracted. Fusion of features from these two structures is conducted based on the recent findings suggesting that $\mathrm{Cx}$ changes in autism are related to CWM abnormalities. Also, when fusing features from more than one structure, this would increase the robustness of the CAD system. Moreover, fMRI experiments are done and analyzed to find areas of activation in the brains of autistic and typically developing individuals that are related to a specific task. All sMRI findings are fused with those of fMRI to better understand ASD in terms of both anatomy and functionality, and thus better classify the two groups. This is one aspect of the novelty of this CAD system, where sMRI and fMRI studies are both applied on subjects from different ages to diagnose ASD. In order to build such a CAD system, three main blocks are required. First, 3D brain segmentation is applied using a novel hybrid model that combines shape, intensity, and spatial information. Second, shape features from both $\mathrm{Cx}$ and CWM are extracted and an fMRI reward experiment is conducted from which areas of activation that are related to the task of this experiment are identified. Those features were extracted from local areas of the brain to provide an accurate analysis of ASD and correlate it with certain anatomical areas. Third and last, fusion of all the extracted features is done using a deep-fusion classification network to perform classification and obtain the diagnosis report. Fusing features from all modalities achieved a classification accuracy 
of $94.7 \%$, which emphasizes the significance of combining structures/modalities for ASD diagnosis.

To conclude, this work could pave the pathway for better understanding of the autism spectrum by finding local areas that correlate to the disease. The idea of personalized medicine is emphasized in this work, where the proposed CAD system holds the promise to resolve autism endophenotypes and help clinicians deliver personalized treatment to individuals affected with this complex syndrome. 
TABLE OF CONTENTS

ACKNOWLEDGMENTS . . . . . . . . . . . . . . . . . iii

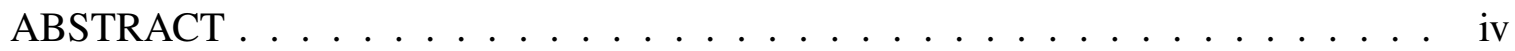

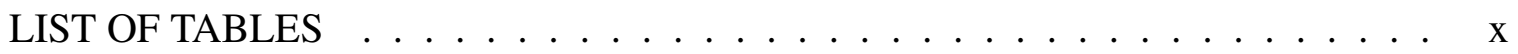

LIST OF FIGURES $\ldots \ldots \ldots \ldots \ldots \ldots \ldots \ldots$ xi

LIST OF ALGORITHMS $\ldots \ldots \ldots \ldots \ldots \ldots \ldots \ldots \ldots$ xiv CHAPTER

I. SURVEY ON AUTISM DIAGNOSIS USING MAGNETIC RESONANCE

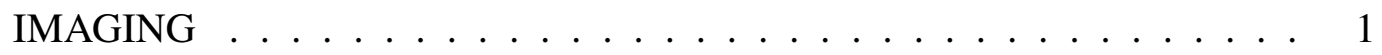

A. Problem Statement $\ldots \ldots \ldots \ldots \ldots \ldots \ldots$

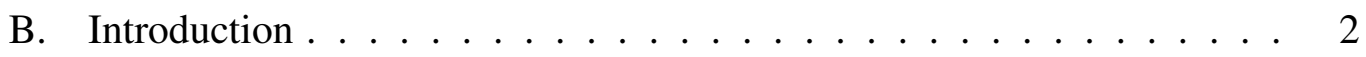

C. Studying ASD with sMRI $\ldots \ldots \ldots \ldots \ldots \ldots$

1. Studying ASD impacts on anatomical structures with sMRI $\ldots 8$

a. Cerebral cortex ................. 8

b. Posterior cranial fossa (cerebellum, vermis, and brain stem) . 13

c. Amygdalae . . . . . . . . . . . . . . . . . . . . 14

d. Hippocampus . . . . . . . . . . . . . . . 16

e. Corpus callosum $\ldots \ldots \ldots \ldots \ldots \ldots$

f. Thalamus, caudate, and putamen $\ldots \ldots \ldots \ldots$

g. Total brain volume and head circumference . . . . . . . 18 
D. Studying ASD with DTI $\ldots \ldots \ldots \ldots \ldots \ldots$

1. DTI: Basic concepts _ . . . . . . . . . . . . . . . . 19

2. Studying ASD impacts on anatomical structures with DTI . . . 24

a. Whole-brain VBA . . . . . . . . . . . 25

b. Tract-based spatial statistics (TBSS) . . . . . . . . . . 28

c. ROI analysis . . . . . . . . . . . . . . . 31

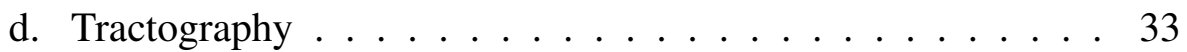

e. Classification-based analysis . . . . . . . . . . . . . . 38

E. Studying ASD with fMRI . . . . . . . . . . . . . . . . . . 39

F. Limitations of existing work $\ldots \ldots \ldots \ldots \ldots$

G. Motivation behind this work . . . . . . . . . . . . . 48

H. Dissertation Layout . . . . . . . . . . . . . . . . . . . . 49

II. SEGMENTATION OF MR BRAIN IMAGES USING ADAPTIVE SHAPE PRIOR AND HIGHER-ORDER MGRF . . . . . . . . . . . . . . . 51

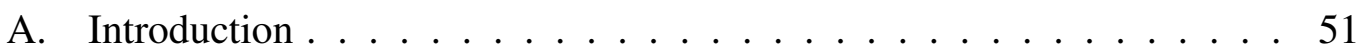

1. Probabilistic segmentation . . . . . . . . . . . 53

2. Atlas-based segmentation $\ldots \ldots \ldots \ldots \ldots$

3. Deformable models-based segmentation . . . . . . . . . . . 59

B. Methods . . ....................661

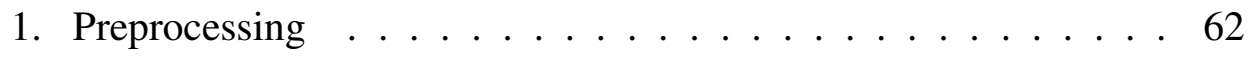

2. Joint MGRF Model of MR Brain Images $\ldots \ldots$. . . . . . 63

a. Adaptive Shape Model $P_{\mathrm{sp}}(\mathbf{m}) \ldots \ldots 63$

b. First-Order Intensity Model $P(\mathbf{g} \mid \mathbf{m}) \quad \ldots \ldots \ldots$ 
c. MGRF Model With Second- and Higher-order Cliques $P_{\mathbf{V}}(\mathbf{m}) 65$

C. Experimental Results . . . . . . . . . . . . . . . . 67

D. Summary and Conclusion . . . . . . . . . . . . . . . 69

III. A CAD SYSTEM FOR AUTISM DIAGNOSIS USING STRUCTURAL AND FUNCTIONAL MRI MODALITIES . . . . . . . . . . . . . 77

A. Introduction . . . . . . . . . . . . . . . . 78

B. Methods ........................ 80

1. Segmentation of CWM and Cx from MR images . . . . . . . 80

2. SMRI experiment: shape feature extraction . . . . . . . . . 80

a. Cx shape features ............... 81

b. CWM shape features . . . . . . . . . . . . 82

3. FMRI experiment: monetary reward feedback . . . . . . . 83

4. Deep Fusion Classification Network (DFCN) . . . . . . . . . . . 84

C. Experimental Results . . . . . . . . . . . . . . . . 87

1. Analysis of NCAE weights . . . . . . . . . . . . . 89

D. Summary and Conclusion . . . . . . . . . . . . . . . . 89

IV. CONCLUSIONS AND FUTURE WORK . . . . . . . . . . . . . . . . 99

A. Conclusions . . . . . . . . . . . . . . . . . . . . 99

B. Future Work . . . . . . . . . . . . . . . . 100

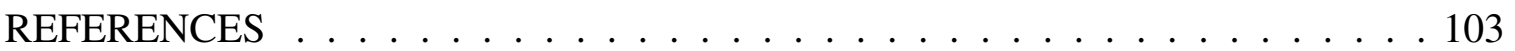

CURRICULUM VITAE . . . . . . . . . . . . . . . . . . . . . . . 144 


\section{LIST OF TABLES}

TABLE.

PAGE

1. Accuracy of the proposed segmentation approach using Dice Similarity Coefficient (DSC) $(\%)$, the modified Hausdorff Distance (MHD), and Absolute Brain Volume Difference (ABVD) (\%) for the IBIS database. Metrics are represented as Mean \pm Standard Deviation. . . . . . . . . . . . . . . . . . 75

2. Accuracy of the proposed segmentation approach using Dice Similarity Coefficient DSC (\%), the modified Hausdorff Distance (MHD), and Absolute Brain Volume Difference ABVD(\%) for the KKI database. Metrics are represented as Mean \pm Standard Deviation. . . . . . . . . . . . . . . . . . . . 75

3. Accuracy of the proposed segmentation approach using Dice Similarity Coefficient DSC (\%), the modified Hausdorff Distance MHD, and Absolute Brain Volume Difference ABVD $(\%)$ for the NYU and UCLA databases. Metrics are represented as Mean \pm Standard Deviation. . . . . . . . . . . . . 76 


\section{LIST OF FIGURES}

FIGURE.

PAGE

1. Brain cerebellum $(C)$, occipital $(O)$, parietal $(P)$, frontal $(F)$ and temporal $(T)$ lobe, caudate (CA), putamen (PU), and thalamus (TH) ROIs (a) for measurements and (b) VBM measurements (right) on cerebral WM lobes of an autistic brain (left) . . . . . . . . . . . . . . . . . . . 7

2. Brain surface measurements using spherical harmonics $(\mathrm{SH}) . \ldots . . . . .7$

3. Color-coded CWM curvature map (a) of a brain mesh and measuring the corpus callossum (CC) surface (b) for autism identification. . . . . . . . . . . . . . 8

4. Isotropic (a) and anisotropic (b) diffusion of a single molecule. . . . . . . . . . 19

5. Diffusion ellipsoid: the orientations (a) and sizes (b) of its principal axes defined by the eigenvectors and eigenvalues of the tensor matrix $D$. . . . . . . . 20

6. DTI-based brain anisotropy and microstructure: the reference non-diffusion image (a) and gray-coded scalar MD (b), FA (c), RA (d), $\lambda_{\|}$(e), and $\lambda_{\perp}$ (f) maps.

7. Reference non-diffusion image (a) and the color-coded fiber orientation map (red, green, and blue correspond to the diffusion $x-, y$-, and $z$-axes respectively).

8. Full brain tractography using the "3D Slicer" software: color-coded main fiber directions. . . . . . . . . . . . . . . . . 23

9. Discrete (black) vs. smoothed continuous (red) tracking (a) and deterministic fiber tracts generated with the "3D Slicer" software in the corpus callosum ROI (colors represent the FA values along the tracts). . . . . . . . . . . . . 24

10. Localizing BOLD effects and correlating them to areas of activation in the brain. 40

11. Example for a T1-weighted MRI for an adult (a) and an infant (b) brain. . . . . 52

12. The proposed segmentation framework. . . . . . . . . . . . . 62 
13. Samples of the second- $(a)$, third- $(b)$, and fourth-order $(c)$ cliques for the 26neighborhood (graph cliques are shown in different colors for visualization pur-

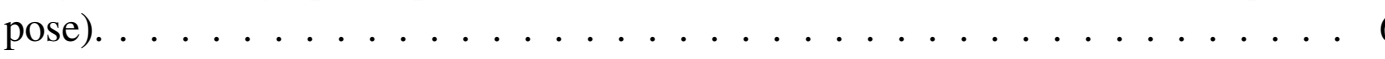

14. Segmentation results projected onto axial, coronal, and sagittal planes for a sample from the IBIS database for infants: (a) original MRIs after skull stripping, and the segmentation using (b) intensity model alone; (c) intensity and shape models (d) intensity, shape, and higher-order MGRF models; and (e) the iBEAT method. Ground truth is shown in (f). CWM is in yellow, GM in blue, and cerebrospinal fluid (CSF) is in red for visualization purposes. All subjects are bias-corrected prior to segmentation using iBEAT or the proposed method. It is clear that the intensity model alone (b) is not sufficient for segmentation, since the contrast is extremely low between CWM and GM. Results were further enhanced using the joint model $(\mathrm{d}) \ldots \ldots \ldots \ldots$

15. Segmentation results projected onto axial, coronal, and sagittal planes for a sample from the KKI database: (a) original MRIs after skull stripping, and the segmentation using (b) intensity model alone; (c) intensity and shape models (d) intensity, shape, and higher-order MGRF models; and (e) the iBEAT method. Ground truth is shown in (f). CWM is in yellow, GM in blue, and cerebrospinal fluid (CSF) is in red for visualization purposes. All subjects are bias-corrected prior to segmentation using iBEAT or the proposed method. . . . . . . . . . . . 71

16. Segmentation results projected onto axial, coronal, and sagittal planes for a sample from the UCLA database: (a) original MRIs after skull stripping, and the segmentation using (b) intensity model alone; (c) intensity and shape models (d) intensity, shape, and higher-order MGRF models; and (e) the iBEAT method. Ground truth is shown in (f). CWM is in yellow, GM in blue, and cerebrospinal fluid (CSF) is in red for visualization purposes. All subjects are bias-corrected prior to segmentation using iBEAT or the proposed method. . . . . . . . . . . 72

17. Segmentation results projected onto axial, coronal, and sagittal planes for a sample from the NYU database: (a) original MRIs after skull stripping, and the segmentation using (b) intensity model alone; (c) intensity and shape models (d) intensity, shape, and higher-order MGRF models; and (e) the iBEAT method. Ground truth is shown in (f). CWM is in yellow, GM in blue, and cerebrospinal fluid (CSF) is in red for visualization purposes. All subjects are bias-corrected prior to segmentation using iBEAT or the proposed method. . . . . . . . . . . 74

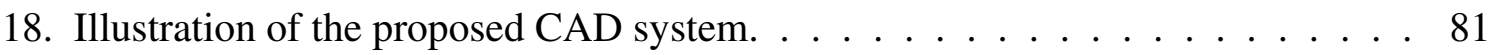

19. Desikan-Killiany Labeling Atlas _ . . . . . . . . . . . . . . . 81

20. (a) Boundary of segmented CWM. (b) First distance map. (c) Second distance map. (d), (e) Extracted CWM gyri. (f) 3D visualization of the extracted gyri. . . 
21. (a) GLM, and (b) the HRF signal. (c) BOLD signal that results from convolving (a) and (b) . . . . . . . . . . . . . . . . . . . . . . . . 91

22. Structure of DFCN. . . . . . . . . . . . . . . . . . . 92

23. Classification of four ASD cases, color coded to indicate the strength of association of each DK area with the ASD phenotype. This emphasizes the significance of personalized medicine. . . . . . . . . . . . . . . . . 93

24. Classification of four ASD cases, color coded to indicate the strength of association of each DK area with the ASD phenotype. This emphasizes the significance of personalized medicine. . . . . . . . . . . . . . . . . . 93

25. Classification of four ASD cases, color coded to indicate the strength of association of each DK area with the ASD phenotype. This emphasizes the significance of personalized medicine. . . . . . . . . . . . . . . . . 94

26. Classification of four ASD cases, color coded to indicate the strength of association of each DK area with the ASD phenotype. This emphasizes the significance of personalized medicine. . . . . . . . . . . . . . . . . 94

27. (a) Original input CDF vector of one of the Cx measures, $K_{G}$, to the NCAE. (b) reconstructed $\mathrm{CDF}$ vector from the NCAE. (c) reconstruction error between both. 95

28. (a) Original input vector of the fMRI signal to the NCAE. (b) reconstructed vector from the NCAE. (c) reconstruction error between both. . . . . . . . . . 96

29. (a) Original input CDF vector of one of the CWM measures, $S$, to the NCAE. (b) reconstructed $\mathrm{CDF}$ vector from the NCAE. (c) reconstruction error between both. . . . . . . . . . . . . . . . . . . . . . . 97

30. (a) Original input CDF vector of one of the $\mathrm{Cx}$ measures, $K_{N}$, to the NCAE. (b) reconstructed $\mathrm{CDF}$ vector from the NCAE. (c) reconstruction error between

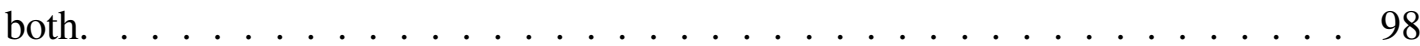




\section{LIST OF ALGORITHMS}

\section{ALGORITHM.}

PAGE

1. Steps of the Proposed segmentation framework . . . . . . . . . 73 


\section{CHAPTER I}

\section{SURVEY ON AUTISM DIAGNOSIS USING MAGNETIC RESONANCE IMAGING}

This chapter reviews recent applications of structural magnetic resonance imaging (sMRI), diffusion tensor imaging (DTI), and functional MRI (fMRI) to study autism spectrum disorder (ASD). Main reported findings are sometimes contradictory due to different age ranges, hardware protocols, population types, numbers of participants, and image analysis parameters. The primary anatomical structures, such as amygdalae, cerebrum, and cerebellum, associated with clinical-pathological correlates of ASD are highlighted through successive life stages, from infancy to adulthood. This survey demonstrates the absence of consistent pathology in the brains of autistic children and lack of research investigations in patients under two years of age in the literature. The known publications also emphasize advances in data acquisition and analysis, as well as significance of multimodal approaches that combine resting-state, task-evoked, and sMRI measures. Reported findings show good promise towards the early and non-invasive ASD diagnostics.

\section{A. Problem Statement}

Autism is a neuro-developmental disorder that has many symptoms, most prominently, social impairment and repetitive behaviors. Autism is typically diagnosed at the age of three; however, some characteristics can sometimes be observed at around 12 months of age [1]. The main cause of autism is still unknown; however, it is believed that genetic factors are responsible [1].

Most of the current existing methods for autism diagnosis are subjective. Pediatricians often identify autism at about three years of age [2]. Several screening exams 
can be applied to children at two years of age or older. Some of these exams include the DSM-V [3], Autism Diagnostic Observations Schedule (ADOS) [4], and Autism Diagnostic Interview Revised (ADI-R) [5]. Most of these exams require having an IQ exam as well, e.g. the Wechsler Intelligence Scale for Children (WISC) [6]. A diagnosis involves individual examinations from child psychiatrists, behavioral and occupational therapists, and speech language pathologists. Yet as mentioned earlier, all of these exams provide subjective evaluations and cannot be relied upon for providing an accurate and a quantified diagnosis.

In order to diagnose autism objectively, medical imaging modalities have been largely incorporated recently. Magnetic resonance imaging (MRI) is the one of the most associated modalities with such disorder due to its many advantages such as the high contrast and spatial resolution. Moreover, MRI modalities have emerged as powerful means that facilitate non-invasive clinical diagnostics of various diseases and abnormalities since their inception in the 1980s. Multiple modalities, such as different types of the structural MRI and diffusion tensor imaging (DTI) have been employed to investigate facets of ASD in order to better understand this complex syndrome. Extracting significant features from such modalities that would classify autistic and control brains remains a challenging task, especially with infant brains.

\section{B. Introduction}

The term "autism spectrum disorder" (ASD) refers to a collection of neuro-developmental disorders that affect linguistic, behavioral, and social skills. Autism has many symptoms, most prominently, social impairment and repetitive behaviors. The most severe form of the ASDs is autistic disorder (AD), while milder forms include Asperger syndrome (ASP), childhood disintegrative disorder, and not-otherwise-specified pervasive developmental disorder (NPDD). By some estimates, the ASD affects one out of 68 eight years of age, with males being four times more likely to develop it than females. About $30 \%$ 
of children with ASD have epilepsy at later stages [1]. Autism is typically diagnosed at the age of three; however, some characteristics can sometimes be observed at around 12 months of age. What causes autism is yet unknown; however, it is mainly believed that genetic and environmental factors in complex combinations are responsible [1]. No current cure is specifically designed for autism. However, educational, behavioral, or skill-oriented therapies were designed to remedy specific symptoms in each individual. Such therapies can result in substantial improvement, particularly when started at a young age. Conventional autism diagnostics rely on recording the patient's reactions to varied stimuli through periodic screening interviews. Early observations by parents can greatly reduce the false positive rate and circumvent unnecessary referrals [7]. However, the diagnosis is subject to human observational and perceptual errors because current diagnostic standards for autism rely on subjective behavioral screenings A more objective computer aided diagnosis (CAD) is a prime necessity in this field.

Recent advances in neuropathological and neuro-imaging studies have revealed a great deal concerning the pathogenesis of autism and suggest new non-invasive ways to automate autism detection by revealing differences between quantitative characteristics of typically developing and autistic brains. Increases in white matter volume and brain weight in postnatal development are proportionate as myelin contributes a significant percentage of any brain weight change during the first years of life. Abnormalities in brain size with widespread increases in both gray and white matter volumes suggest that the underlying pathology in autism consists of widely distributed neuroanatomical changes. In addition to changes in brain size, other gross morphological features of the autistic phenotype include polymicrogyria, macrogyria and schizencephaly [8]. These changes imply a disturbance in cell migration during the early stages of corticogenesis. More recently, The study by Bailey et al. [9] described postmortem evidence of neocortical abnormalities in the brains of 4 out of 6 examined autistic patients, i.e. laminar disturbances and heterotopias. The four cases were megalencephalic. This was the first study to advocate the presence of 
pathology in widespread, disparate areas of cortex and/or their connections, as opposed to isolated anatomical structures. The findings offered a better account for both the seizures and higher-order cognitive deficits found in autism.

The available neuropathological and structural imaging data suggest that autism is the result of a developmental lesion capable of affecting brain growth. One possible explanation for this is the recent finding of minicolumnar abnormalities in autism (i.e., minicolumns of reduced size and increased numbers) [10]. In this initial study, measures of minicolumnar morphometry were obtained on pyramidal cell arrays in nine autistic cases and an equal number of controls. The feature extraction properties of the algorithms were corrected for minicolumnar fragments, curvature of the tissue section, and 3D proportions (stereological modeling) [11]. Later on, the same patient population was used to confirm the presence of cortical radial abnormalities in a study using the Grey Level Index (GLI) ratio, i.e., area covered by Nissl-stained to unstained elements in postmortem samples [12].

These studies were all at the microscopic level, where abnormalities in minicolumnar arrangements of the neurons were found in autistic brains. Since the minicolumn reiterates itself millions of times throughout the brain, those variations were predicted to result in macroscopic changes as well. This encouraged researchers to investigate those changes using several imaging modalities. After the advent in the nineteen eighties, MRI soon became one of the most promising non-invasive modalities for visualization and diagnostics of ASD-related abnormalities. Along with its main advantage of no exposure to radiation, high contrast, and spatial resolution, the recent advances to MRI modalities have notably increased diagnostic certainty. The modalities, being most helpful for studying ASD, include structural MRI (sMRI) [13-24], diffusion tensor imaging (DTI) [25, 26], and functional MRI (fMRI) [27, 28].

In applications to ASD, sMRI helps in investigating structural brain changes in autistic subjects. Many scan sequences of the sMRI are volumetric, i.e., allow for measuring specific brain structures to calculate tissue volumes. In spite of diagnostic abilities 
found for the sMRI, the earlier published results obtained for a limited number of subjects were often contradictory. Specific pulse sequences employed in the sMRI help to reveal different properties of normal and abnormal brain tissues. Modifying the pulse sequence parameters, such as repetition time (TR) and echo time (TE), may emphasize the contrast between gray matter (GM) and white matter (WM), e.g., in the T1-weighted sMRI with short TR and TE, or brain tissue and cerebrospinal fluid (CSF), e.g., in the T2-weighted sMRI with long TR and TE.

The recent DTI characterizes three-dimensional (3D) diffusion of water molecules in a biological tissue $[29,30]$. The DTI has a wide range of clinical applications. In particular, it is used to examine normative white matter (WM) development, neurodevelopmental disorders, and neurodegenerative disorders, e.g., autism, and amyotrophic lateral sclerosis [31].

In neurological studies, where each patient's status can be assessed, the chosen imaging modality should be able to clearly demonstrate the abnormalities. The conventional MRI lacks sensitivity in distinguishing the abnormalities on an individual-subject basis [26], whereas the DTI has the potential to reveal such abnormalities. This new information comes from better image contrast, more detailed WM morphology, refined anatomical locations, and more accurate connectivity analysis [26]. Additional DTI offers many contrast-related measurements, including the widely used fractional anisotropy (FA) and estimates of shapes and sizes of specific WM tracts, i.e., WM morphology [26]. Moreover, DTI provides superior anatomical information for clearer identification of areas with WM abnormalities [25]. It also provides unique brain connectivity measurements via 3D fiber reconstruction, e.g., tractography, [32].

Many neuroimaging studies also attempted to focus on connectivity analysis in autism. These types of studies can be referred to as functional magnetic resonance imaging (fMRI). fMRI aims at localizing task-evoked blood oxygen level-dependent (BOLD) effects in the brain, and subsequently analyzing those effects in order to find anatomical ar- 
eas of activation related to that specific task. This survey presents applications of the sMRI, DTI, and fMRI to study the ASD (mostly, for the last two decades) in order to outline the most important findings with these modalities across all life stages. This provides a comprehensive study unlike the recent surveys that either focus on one modality, [33-36], or address a specific life stage, $[35,37]$. Moreover, this survey highlights the methodologies conducted in each study and categorizes them with respect to the approach (e.g. whether they are volumetric-based, or surface-based in sMRI), and user's intervention (manual, automated, semi-automated).

The survey is structured as follows: sections I.C, I.D and I.E below address the use of the sMRI, DTI, and fMRI respectively. Findings in the reviewed publications emphasize benefits for studies of the ASD, as well as other medical abnormalities, by acquiring and analyzing complementary multi-modality data.

\section{Studying ASD with sMRI}

The earlier findings of the 1980 s-1990s $[8,9,13-24,38-78]$ have many contradictions. However, the much better spatial resolution and contrast of the recent advanced MRI technology made the recent findings of the 2000s-2010s more consistent [79-81]. This survey focuses on the latter studies and attempts to classify the reported abnormalities at different life stages for each of the autism-related anatomical structures.

Cross-sectional or longitudinal sMRI scans, collected at different time instants for the same individual, are studied by either region-of-interest (ROI) based volumetry, or surface-based morphometry (abbreviated as RBV, and SBM respectively). The $\boldsymbol{R} \boldsymbol{B} \boldsymbol{V}$ usually focuses on the total volume or area measures for a chosen region, but requires manual intervention by experts to delineate it. This age-sensitive and time consuming process depends on level of automation, yet is powerful in a statistical sense. A method that is correlated to RBV and is known as voxel-based morphometry, $\boldsymbol{V B} \boldsymbol{M}$, targets tissue density, e.g., relative GM concentration, or volume, e.g., regional volume differences of a certain 

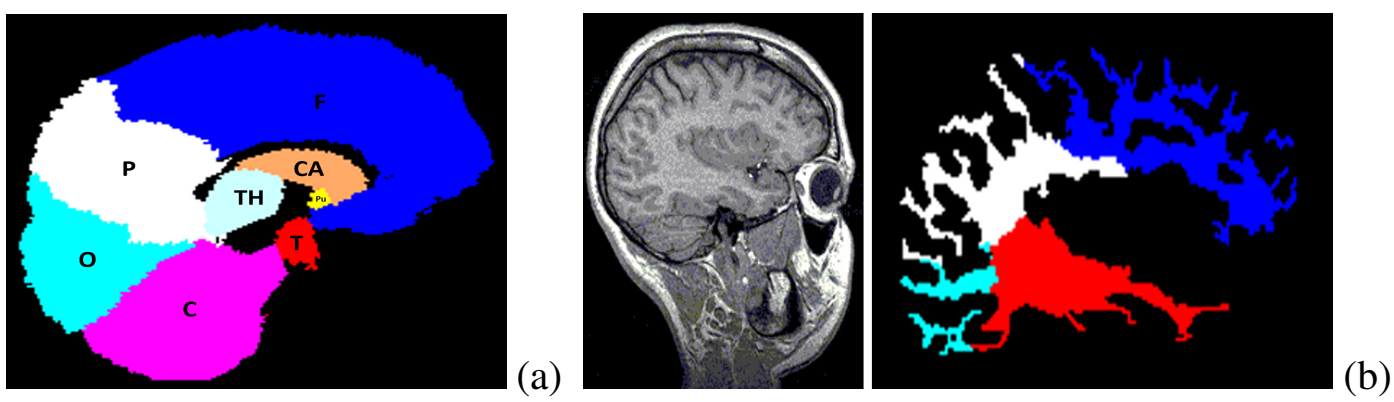

FIGURE 1: Brain cerebellum (C), occipital (O), parietal (P), frontal (F) and temporal (T) lobe, caudate (CA), putamen (PU), and thalamus (TH) ROIs (a) for measurements and (b) VBM measurements (right) on cerebral WM lobes of an autistic brain (left).

tissue. The $\boldsymbol{S B M}$ addresses topological shape features, like surface curvature and folding degree, that cannot be obtained directly using the RBV on a brain SMRI. The SBM is applied mostly to the cerebral cortex, along with its lobes and gyrification patterns.

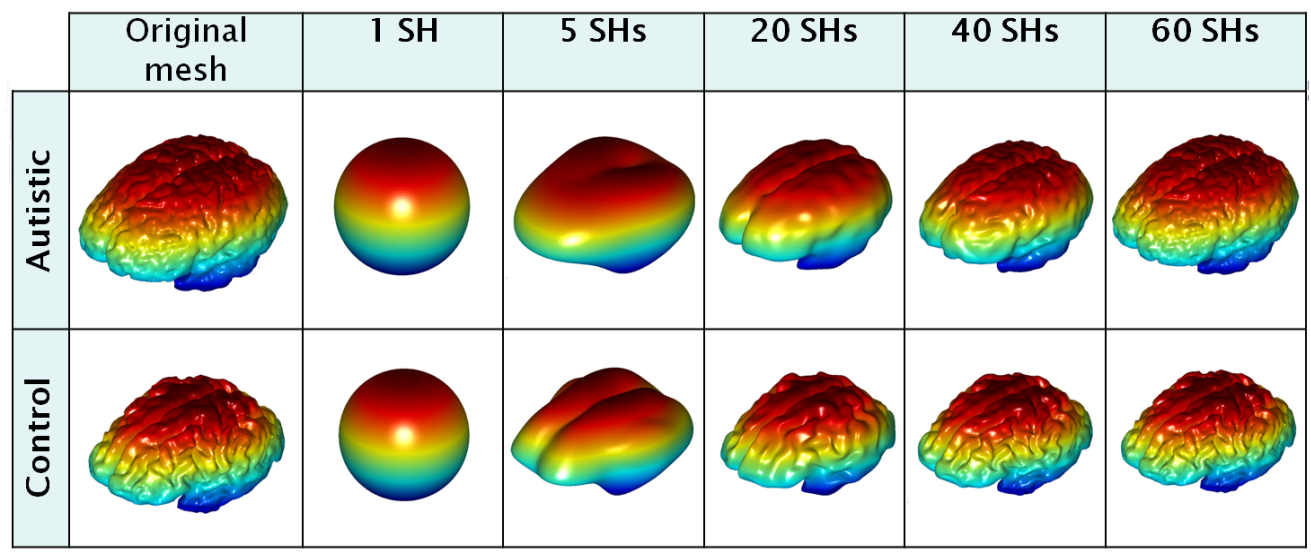

FIGURE 2: Brain surface measurements using spherical harmonics (SH).

In the brain structures, such as corpus callosum, the SBM computes the surface area and centerline changes (Figure 3 (b)). The intrinsic cerebral cortex topology is usually described with shape indices, such as the mean curvature $(H)$ [82], degree of sharpness $(S)$, and curvedness $(C)$ :

$$
H=0.5\left(K_{\max }+K_{\min }\right) ; \quad C=\sqrt{0.5\left(K_{\max }^{2}+K_{\min }^{2}\right)} ; \quad S=\left(K_{\max }-K_{\min }\right)^{2},
$$

where $K_{\max }$ and $K_{\min }$ denote the maximum and minimum principal surface curvature, 


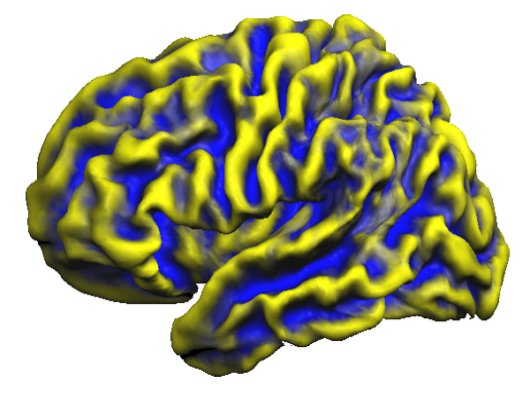

(a)

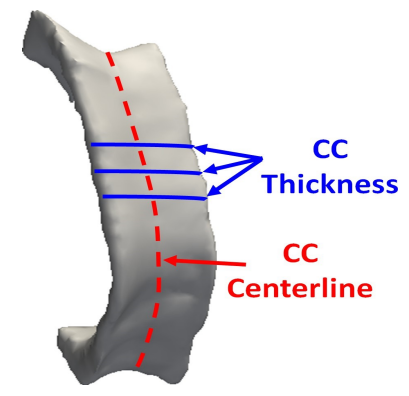

(b)

FIGURE 3: Color-coded CWM curvature map (a) of a brain mesh and measuring the corpus callossum (CC) surface (b) for autism identification.

respectively. The curvedness $C$ characterizes the shape of the voxel neighborhood [82] and can distinguish between highly- and less-folded regions [83]. The folding is also quantified by its sharpness $S$, being proportional to the Willmore integrand.

The ASD studies with the sMRI, including data processing tools and main abnormalities found in brain structures are considered below. Abnormalities found in the cerebral cortex; posterior fossa (vermis, brain stem, and cerebellum); corpus callossum; amygdalae; hippocampus, and thalamus, are addressed at four main life stages: infancy (0-2 years); childhood (3-11 years); adolescence (12-18 years), and adulthood (above 18 years). The measurements also include the total brain volume and head circumference.

1. Studying ASD impacts on anatomical structures with sMRI

a. Cerebral cortex This uppermost brain layer that plays a leading role in human intelligence and perception has been the focus of plenty of sMRI studies on ASD [84]. Changes of cortical thickness and gyrification patterns were analyzed with the SBM, whereas the RBV and VBM measured regional differences in volumes and densities of the cerebral GM and WM.

Infancy According to the longitudinal VBM [85] on 51 autistic children and 25 controls (aged 18-35 months), both the cerebral GM and WM volumes increase in autistic brains. 
The longitudinal RBV on children aged 1.5 to 5 years [86] revealed no changes in the occipital lobe. On the other hand, the cerebral GM volumes were significantly enlarged in the frontal, temporal, and parietal lobes, and in the cingulate gyrus (located in the limbic lobe) of autistic toddlers (aged about 2.5 years). Additionally, there were more abnormal growth profiles for females. The RBV in [87] monitored the total brain volume changes for children aged six months to compare high risk infants to low risk ones with no autistic family members. The fact that cerebrum or lateral ventricle volumes had no significant difference for both groups confirmed earlier findings that the brain enlargement is a postnatal event, occurring around 12 months of age. Another extended longitudinal study [88] pursued the goal of identifying 6-9-month-old infants who might later develop the ASD. It was revealed that the ASD causes an extra-axial fluid and is characterized by excessive CSF over the frontal lobes at 6-9 months of age, which persists at 12-15 and 18-24 months. This leads to large total cerebral volumes that tend to increase with age at a higher rate in males, than females. This finding contradicts the earlier one [86], which showed more enlargement in females. This inconsistency might be caused by manual segmentation, along with the younger ages and the $20 \%$ smaller number of participants in [88].

Childhood Volumetric, voxel-wise, and thickness changes in the cerebral cortex in children with autism have been extensively studied in the literature. In particular, the autistic children aged 2-3 years had 18\% and 12\% larger cerebral cortical WM and GM volumes, respectively, than the control ones, while the older children did not demonstrate such enlargement [89]. The increased frontal and temporal GM and frontal and parietal WM volumes at such a young age (2-4 years) was confirmed in [90]. The increased cerebral volumes in autistic children aged around 4 years and 6 years were found also in [91] and [92], respectively. The latter study reported the increased total cerebral volume, as well as the increased WM and GM volumes for children with both low-functioning autism (LFA) and high-functioning autism (HFA), the total cerebral volume being significantly larger in the 


\section{LFA group.}

The VBM of the GM and WM densities on autistic children around 9 years of age [93] has found a significant decrease in the GM density in the superior temporal sulcus and a decrease in the WM density in the right temporal pole. This finding was supported by [94] where the GM density was found to significantly decrease in the frontostriatal and parietal networks, as well as in the ventral and superior temporal gyrus in autistic children aged around 12 years. The RBV revealed the decreased GM volume in the right lateral orbitofrontal cortex in 10-year-old boys in [95] and the decreased GM volumes in the parietal, left temporal, and left occipital lobes bilaterally in [96]. The left and right frontal lobes of the autistic boys had enlarged $3.6 \%$ and $5.1 \%$, respectively, while all the other lobes grew more significantly.

The cerebral cortices of children with autism were often studied with the SBM as well. The atlas-based SBM in [97] monitored the cortical thickness changes in autistic brains of children aged 10 years. The frontal or occipital lobes did not change, whereas the temporal and parietal lobes had most prominent increases of the total cerebral sulcal and gyral thicknesses. According to the subsequent longitudinal SBM [98] on 10-year old autistic children, with a follow-up scan two years later, the cortical thickness in autistic subjects has decreased in the frontal, temporal, and occipital lobes, comparing to controls. Also, the SBM [99] on 9-year-old children with autism showed the decreased thickness in the right entorhinal, right lateral orbitofrontal, left lateral orbitofrontal, right medial orbitofrontal, left medial orbitofrontal cortex, and right pars triangularis. However, the thickness also increased in the left caudal anterior cingulate cortex and left frontal pole. Significant bilateral differences in sulcal depth in restricted portions of the anterior-insula, frontal-operculum, and in tempoparietal junction, were found in [100]. The study by Gori et al. [101] on 4-year-old males was based on extracting features from GM, WM, and CSF to classify autistic and control brains. Only GM features in different subregions showed a classification performance that reached up to $80 \%$. 
Adolescence Volumetric and voxel-wise cerebral changes have been investigated in a number of publications. The VBM in [102], for example, revealed the increased GM volume in the cortical lobes of 15-year-old autistic males, namely, in the right fusiform gyrus, right temporal and occipital region, and left frontal pole. This work was extended in [103] to investigate changes of the WM volume in 15-year-old autistic males and found that the WM volume decreases at the left middle temporal, right middle frontal, and left superior frontal gyri. The VBM in [104] conducted on subjects with the HFA and ASP showed the lower cortical GM density in the right inferior temporal gyrus, entorhinal cortex, and right rostral tip of fusiform gyrus. The RBV in [105] reported a growth of the cerebral GM in both the HFA and LFA subjects, compared to the controls, but only non-significant changes for people with the ASP. The VBM in [106] on 16-year-old males revealed the lesser WM concentration in the genu, rostrum, and splenium regions in autistic brains, whereas the RBV in [107] on a group of young adolescents has found a larger total cerebral volume of the autistic brains. The lobe volume growth due to the GM volume enlargement was also noticed in the frontal and temporal, but not parietal or occipital lobes. The VBM on autistic and control males around the age of 13 in [108] has found an increase in the GM volume in the parietal lobes, medial and dorsolateral frontal areas, and lateral and medial parts of temporal lobes, as well as a decrease in the WM volume in the frontal, parietal, temporal, and occipital lobes. The longitudinal RBV on 13-year-old adolescents in [109] has found a decelerated WM growth in the frontal, temporal, parietal, and occipital lobes, together with an abnormally accelerated GM expansion in the putamen and anterior cingulate cortex.

To monitor cortical changes in the adolescent groups due to autism, the SBM on a group of 12-year-old autistic and control adolescents was used in [110] to measure changes in the cerebral folding and better investigate the gyrification patterns. As was found, the adolescents had the higher left frontal gyrification index, than the adults, as well as the cortical folding had decreased bilaterally with age in all the autistic subjects, but not in the controls. The SBM on a wide variety of patients including the LFA, HFA, and ASP 
subjects aged 11-13 years in [111] has found for the LFA subjects prominent shape abnormalities centered on pars opercularis of the inferior frontal gyrus, associated with sulcal depth differences in the anterior insula and frontal operculum. The bilateral shape abnormalities in the HFA group were similar to those of the LFA group, but smaller in size and centered more posteriorly in and near the parietal operculum and ventral postcentral gyrus. The ASP group had the correlated with age bilateral abnormalities in the intraparietal sulcus. All these cortical shape abnormalities were more pronounced in the children than in the adolescents. Two successive longitudinal studies on the same group of subjects when their mean age was 17.4 and 19 years, respectively, in [112] demonstrated an accelerated cortical thinning in the autistic brains with respect to the controls in two areas in the left hemisphere, namely, in the posterior portion of the ventral temporal cortex and the superior parietal cortex. This acceleration has happened only for the older adolescents and young adults of the second study.

Adulthood The RBV on 16 autistic males of the average age of 22 years in [113] revealed the larger mean cerebral and third ventricle volumes in the autistic subjects rather than in the controls. The VBM in [114] gave the larger GM volume in the medial frontal gyri, left precentral gyrus, right postcentral gyrus, and right fusiform gyrus. The more recent VBM [115] showed the decreased GM volume in the medial, temporal, and fusiform regions. The SBM in [110] explored changes of the cerebral folding in 27-year-old males. While the left frontal gyrification index had no changes, the cortical folding decreased in autistic adults, compared to children and adolescents. The SBM in [116] on the autistic, ASP, and NPDD subjects aged around 33 years has shown the decreased thickness in the inferior frontal gyrus, pars opercularis, inferior parietal lobule, superior temporal sulcus, precentral and postcentral gyrus, inferior occipital gyrus, prefrontal cortex, anterior cingulate, medial parietal cortex, supramarginal gyrus, and middle and inferior temporal cortex. The SBM in [117] on 15 autistic 22.7-year-old (on average) males has shown that the thick- 
ness increases in the frontal, temporal, occipital, cingulate, and parietal gyrus, as well as in the fusiform gyri, but decreases in the pre- and post-central gyri and para-central gyrus.

Conclusions According to the cited studies, the cerebral cortex starts changing in the autistic brains at the age of around 12 months, and no significant differences could be monitored earlier, except of the recent findings in [88]. The cerebrum changes of an autistic brain at such an early age include the age-proportional enlargements of the lobes and increases of the cortical WM and GM. The growing cerebral volume, including the GM and WM, in early childhood indicates simultaneously the decreasing GM and WM density. Also, children with autism demonstrate significant changes in the cortical thickness. These volumetric and thickness differences continue to increase at the later stages of life.

b. Posterior cranial fossa (cerebellum, vermis, and brain stem) This bowlshaped cavity in the skull includes the rearmost brain part, called the cerebellum, which is located just above the brain stem. The latter provides for mental activities and includes pons, midbrain, and medulla oblongata. The cerebellum mainly coordinates body movements, e.g., keep equilibrium and balance [84]. Posterior cranial fossa has also been investigated in the literature for any correlates with ASD at different life stages.

Childhood Area measurements on 22 autistic children in [118] showed no abnormalities in the total vermis, vermis lobules VI-VII, pons, and midbrain, which could be related to autism. However, the decreased WM density in the cerebellum of children with autism was reported in [94] and [93], and the larger by 39\% cerebellar WM volume was found in [89] for 2-3-year-old children with autism compared to controls. Autistic boys had the lesser GM and smaller GM-to-WM ratios and vermian lobules than the normal ones. The reduced cross-sectional areas of the vermis lobules VI-VII in autistic children were also reported in $[89,119,120]$. The larger cerebellar WM and GM volumes and increased area of the anterior and posterior cerebellar vermis were found in the 6-year-old children with the LFA, HFA, and NPDD in [92]. 
Multiple brain stem volume studies gave contradicting conclusions. The early areal measurements in $[18,66]$ claimed reduced brain stem size, whereas the subsequent works, such as $[39,41,42,49,118]$ found no significant differences between the autistic and control groups. No differences in the brain stem volumes for these groups were found also in $[121,122]$. However, the recent study [123] of 10-year-old children, with a follow-up after two years, came up with different findings: the brain stem volume was stable in the controls over the two-year period, but the increased GM volume of an autistic brain implied the larger entire brain stem volume, so that the autistic brain volumes eventually became comparable to those of the 15-year old controls.

Adolescence and adulthood An increase in the GM volume in the cerebellum in [108] is consistent with the studies of children. The cerebellar volumes of autistic children and adolescents are also consistent with those of adults. As shown in [121] on 22-year-old subjects, the total cerebellar volume and cerebellar hemispheres are larger in the autistic group both with and without correction by the total brain volume. But the volumetric and area measurements of the vermis and brain stem did not differ significantly between the autistic and control groups. Also, the lower GM density in the frontostriatal and cerebellar regions, along with the widespread WM differences, had been reported in [124] and the lower brain stem and total brain stem GM volumes in the autistic adults have been found in [125].

Conclusions The posterior fossa structures are significantly affected with the ASD from an early age of two years and during all the subsequent life stages. Some inconsistencies in the brain stem abnormalities found might be caused by wide age and gender differences of the participants.

c. Amygdalae This structure is located in the temporal lobes of the brain and contains nuclei that stimulate responses to different actions, e.g., fear and threat [84]. Its major role in such important brain activities attracted researchers towards finding any cor- 
relates with the ASD.

Infancy So far, the amygdalar changes in the infancy period are not studied, and the youngest subjects in such studies are around three years old.

Childhood The RBV on 29 autistic and 26 control subjects (the average age of 3.9 years) in [91] has shown that in the autistic subjects the amygdalae are enlarged proportionally to the overall increase of the entire cerebral volume. The more recent volumetry [126] on a larger group of the 45 autistic subjects suggested the enlargement of only the right amygdalar volume at the ages of 3-4 years. The longitudinal RBV in [127], which was performed in order to precisely define the age at which the amygdalae begin to enlarge, started with the 85 autistic subjects aged 37 months on average, and had the follow-up scan one year later on 45 of these autistic subjects. That enlarging the amygdalar volume was found in both the cases, although with a higher rate for the latter group, confirms that this enlargement is present at such an early age of around three years. The volumetry on an older population (7.5-12.5 years) in [128] demonstrated that both the right and left amygdalae volumes were enlarged. However, the longitudinal RBV in [129], which involved 15 autistic subjects aged 10.6 years on average and their follow-up scan after reaching adolescence, has found no significant difference between the left, right, or total amygdalar volumes for the autistic and control subjects. This contradiction to all other studies might be caused by the relatively small population used.

Adolescence No significant difference in left, right, or total amygdalae volumes in the adolescents was found in various studies, e.g., [128, 129].

Adulthood The RBV in [130] explored the hypothesis that parents of autistic children would show similar structural changes. This study was conducted in part on the amygdalar volumes of 15 autistic subjects (the average age of 30.3 years), 17 controls (the average 
age of 43.6 years), and 17 parents of autistic children. The amygdalae were smaller in the autistic group than in the other two groups.

Conclusions The amygdalar volumes generally increase in autistic brains at early stage of life, with the rate of increase being proportional to the age (the known studies started from the 3-year-old subjects). By adolescence, no significant differences in this structure could be found. However, at the subsequent life stages its volume decreases in autistic brains.

d. Hippocampus This part of the cerebral cortex interlocks with the dentate gyrus and is mainly concerned with memory actions and passing information from shortterm to long-term memory areas [84]. Hippocampus also proved to have some correlates with the ASD from a young age.

Infancy Similar to the amygdalae, no hippocampal changes have been explored at this period, with the youngest subjects age having been around $2.5-3$ years.

Childhood The enlarged hippocampus was found in autistic children at the ages of both 3-4 [91] and 7.5-12.5 years [128], especially at the HFA group in the latter study. The enlargement only in the right hippocampus in children aged around 10 years was reported in [129]. The earlier study [131] suggested that the area dentata in autistic subjects was significantly smaller than in the normal ones, with the largest deviation at the ages from 29 months to four years.

Adolescence The hippocampus was found enlarged in autistic subjects in [128]; however, this finding differs from the more recent results in [129], where the right hippocampal volume has increased in the autistic children, but not in the adolescents. In the latter case, it was found that differences between hippocampal volumes in autistic and control brains became insignificant with time. 
Adulthood The hippocampus of autistic adults was confirmed to be significantly enlarged [130], compared to controls and also to parents of autistic children. The left hippocampus was larger in both the parents of autistic children and the adults with autism, compared to the controls. The VBM in [114] has shown an increase in the GM volume of the left hippocampus.

Conclusion In autism, the hippocampus is enlarged at all ages.

e. Corpus callosum This largest WM structure in the brain connects the left and right hemispheres and passes information between them [84]. Corpus callosum is found to be correlated with the ASD. In particular, the two successive longitudinal RBV studies [132] on 19 subjects with autism and four subjects with NPDD (first, at the age of 10.6 years and then 13.1 years on average) have found persistent reductions in the total corpus callosum volumes in the autistic subjects compared to the healthy controls. Only the size of rostral body subdivision has normalized over time. The centerline length of the corpus callosum was significantly reduced in young adults with autism compared to controls [133].

Conclusion The corpus callosum is reduced in size in the ASD.

f. Thalamus, caudate, and putamen The symmetrical thalamus is responsible for preprocessing information coming from other parts of the brain. The caudate nucleus, along with the putamen, form the basal ganglia of the brain, are called the dorsal striatum, and are heavily involved in motor control [84].

The GM volume decreases in the right thalamus of adolescent males with autism and the thalamic volume decreases in adults with autism compared to controls, as was found, respectively, in [102] and [134]. However, no volume differences of the basal ganglia, caudate, or putamen at all ages have been found in [135]. The more recent RBV by the same research group [136] found no differences in both the right and left thalamic nuclei between the 19-year-old, on average, control and autistic groups. Studying the older adults 
(aged 28 years on average) showed that the right caudate nucleus has a larger volume in the autistic brain [137].

g. Total brain volume and head circumference Changes in the total brain volume and head circumference have been associated with the ASD in several publications.

Infancy As shown in [85] for 18-35 month-old infants, the total brain volume increases in the group with autism and their normal at birth head circumference becomes significantly larger around 12 months of age compared to the healthy group. This finding was confirmed in [87], showing no significant difference in the head circumference at six months of age in infants with high risk of autism.

Childhood The head circumference grows also in children with autism. The increased brain volume and head circumference in a large group of 10-year-old children with autism have been confirmed in [138] and supported in other studies, e.g., in [122] on 9-year-old autistic males and in [90]. However, a more recent publication [97] reported insignificant increases in the total brain volumes for children with autism (this contradiction to the above findings might be due to a small number of participants). The later stages of life show no difference between the autistic and neurotypical groups in the total brain volumes, probably because the growth rate of the normal brains increases at these stages.

\section{Studying ASD with DTI}

DTI characterizes 3D diffusion of water molecules in a biological tissue with DT [29, 30]. The DTI is widely used in clinical applications, e.g., to examine a normative WM development, neurodevelopmental disorders, like ASD, and neurodegenerative disorders, such as amyotrophic lateral sclerosis [31]. For completeness, a review of the basic principles of DTI follows. 


\section{DTI: Basic concepts}

Water diffusion in biological tissues has nothing in common with physiological motions and occurs due to a thermal-energy-caused random motion of water molecules, also called an intra-voxel incoherent, or Brownian motion [26]. This diffusion that occurs inside, outside, around, and through cellular structures is hindered by cellular membranes yielding more tortuous paths. The diffusion tortuosity could be amplified by cellular swelling or increased cellular density. Moreover, the membranes tend to restrict intracellular water, and both the hindered and restricted diffusion decrease the apparent water diffusivity [139].

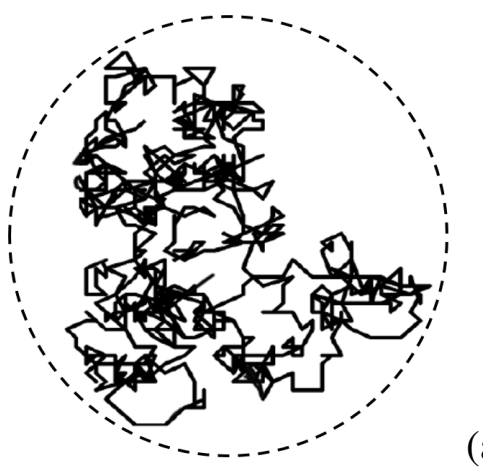

(a)

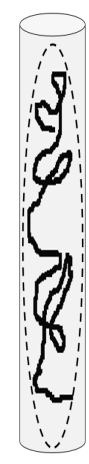

(b)

FIGURE 4: Isotropic (a) and anisotropic (b) diffusion of a single molecule.

An unconstrained medium, such as the CSF, ensures the isotropic diffusion where the water molecules move in a similar manner in all directions. The isotropic diffusion is typical, e.g., for the brain ventricles, whereas the molecular diffusion in the WM is constrained by spatial orientations of the WM tracts. The molecules can diffuse along a fiber track more freely than across it, so that the diffusion becomes anisotropic with a privileged direction. As a result, water diffusion patterns for the brain tissues provide information about the underlying anatomical structures [26].

Generally, a fiber is oriented arbitrarily and has different diffusion coefficients along different directions. Thus, unlike the isotropic diffusion specified not a single scalar diffusion coefficient, the anisotropic one is characterized with a tensor, such that its matrix 
$D$ defines variances and covariances of the 3D diffusion displacements normalized by the diffusion time:

$$
D=\left(\begin{array}{lll}
d_{x x} & d_{x y} & d_{x z} \\
d_{y x} & d_{y y} & d_{y z} \\
d_{z x} & d_{z y} & d_{z z}
\end{array}\right)
$$

The matrix $D$ is symmetric, $d_{y x}=d_{x y}, d_{z x}=d_{x z}$, and $d_{z y}=d_{y z}$, and has six degrees of freedom, which relate to the diffusion strength and spatial orientation. Therefore, the anisotropic diffusion is fully characterized by a diffusion ellipsoid with six independent parameters obtained by diagonalizing the matrix $D$ of Eq. (2): the resulting eigenvectors, $\widehat{\mathbf{v}}_{1}, \widehat{\mathbf{v}}_{2}$, and $\widehat{\mathbf{v}}_{3}$, and their eigenvalues, $\lambda_{1}, \lambda_{2}$, and $\lambda_{3}$, define the orientations and lengths of the longest, middle, and shortest axes, respectively. Physically valid ellipsoids (Figure 5) call for the non-negative eigenvalues.
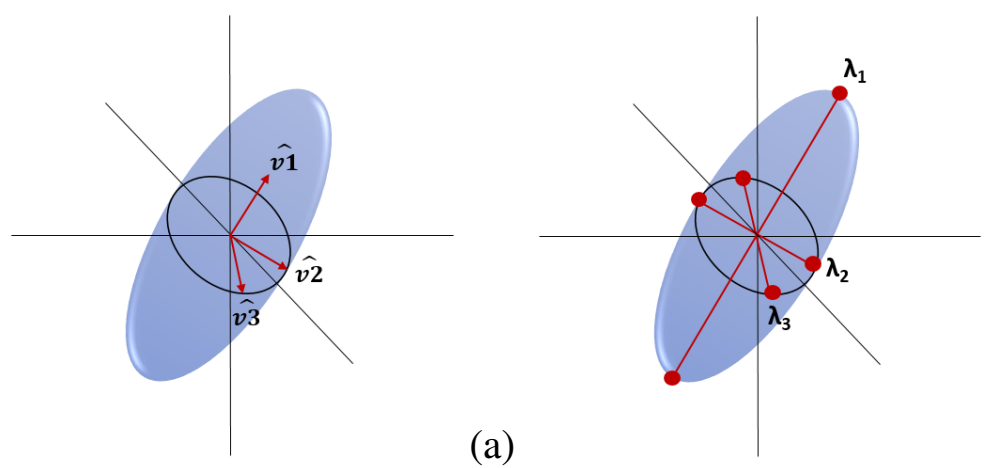

(a)

FIGURE 5: Diffusion ellipsoid: the orientations (a) and sizes (b) of its principal axes defined by the eigenvectors and eigenvalues of the tensor matrix $D$.

For an isotropic medium, the diffusion ellipsoid becomes a sphere: $\lambda_{1}=\lambda_{2}=\lambda_{3}$. For a purely linear anisotropic medium, $\lambda_{1}>0$ and $\lambda_{2}=\lambda_{3}=0$, and the ellipsoid reduces to a line along the $\widehat{\mathbf{v}}_{1}$ direction. A planar anisotropic medium, being isotropic on the plane: $\lambda_{1}=\lambda_{2}>0$ and $\lambda_{3}=0$, reduces the ellipsoid to an oblate.

DT is usually specified with certain parameters at each voxel, that are usually converted into maps of scalar diffusion measurements to facilitate interpreting the voxel-wise 
DTI data. The most common anisotropy and microstructural measurements include fractional (FA) and relative (RA) anisotropy together with mean (MD), axial $\left(\lambda_{\|}\right)$, and radial $\left(\lambda_{\perp}\right)$ diffusivity.

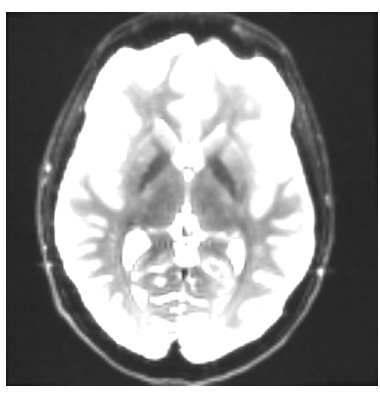

(a)

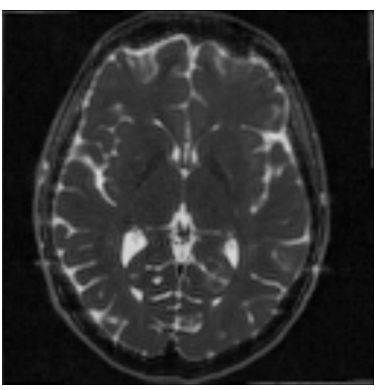

(b)
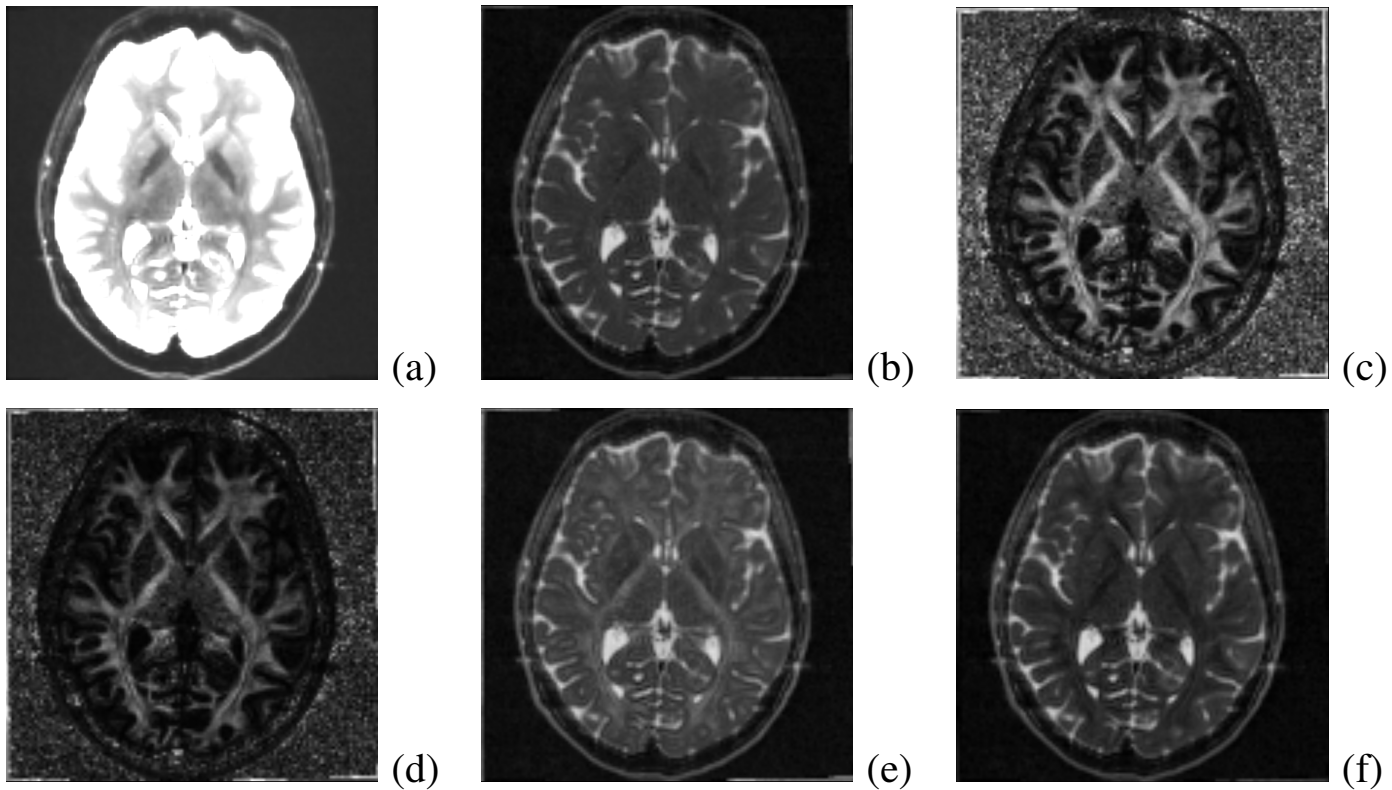

FIGURE 6: DTI-based brain anisotropy and microstructure: the reference non-diffusion image (a) and gray-coded scalar MD (b), FA (c), RA (d), $\lambda_{\|}$(e), and $\lambda_{\perp}$ (f) maps.

The orientation-independent mean diffusivity, also called the trace, measures an overall diffusion in a voxel or region.

$$
\mathrm{MD}=\frac{1}{3}\left(\lambda_{1}+\lambda_{2}+\lambda_{3}\right)
$$

A slightly different MD definition has been used to measure the diffusion descent in brain ischemia [140]. Since the MD values in the CSF are higher than it is in other types of brain tissues, the MD is recommended for the CSF-related disease studies [141].

The fractional anisotropy, described first in [142], is the most popular rotationally invariant (i.e., orientation-independent) measure of how isotropic is the voxel- or regionwise diffusion.

$$
\mathrm{FA}=\sqrt{\frac{3\left[\left(\lambda_{1}-\mathrm{MD}\right)^{2}+\left(\lambda_{2}-\mathrm{MD}\right)^{2}+\left(\lambda_{3}-\mathrm{MD}\right)^{2}\right]}{2\left[\lambda_{1}{ }^{2}+\lambda_{2}{ }^{2}+\lambda_{3}{ }^{2}\right]}}
$$


The FA of a physically realizable diffusion with non-negative eigenvalues ranges from 0 to 1 in the opposite extreme complete isotropic and linear anisotropic cases, respectively. For example, the WM appears whiter due to its higher FA. The reduced FA values usually indicate changes in myelination or degraded axonal structures of the WM [143].

The relative anisotropy is similar to the FA and takes the range between 0 (the complete isotropy) to $\sqrt{2}$ (the complete, i.e., linear anisotropy).

$$
\mathrm{RA}=\sqrt{\frac{\left(\lambda_{1}-\mathrm{MD}\right)^{2}+\left(\lambda_{2}-\mathrm{MD}\right)^{2}+\left(\lambda_{3}-\mathrm{MD}\right)^{2}}{3 \mathrm{MD}^{2}}}
$$

The RA is also defined as the ratio of anisotropic and isotropic parts of the diffusion [144].

The axial, or parallel diffusivity, $\lambda_{\|}$, measures the diffusion along the principal axis (parallel to axons), whereas the radial, or perpendicular diffusivity, $\lambda_{\perp}$, averages the diffusion along the two minor axes to measure the degree of restriction due to membranes and other effects.

These two measurements are closely connected to the WM pathology [139] and have been used to observe developmental and pathological fiber alterations, e.g., to study dysmyelinating disorders [145].

Main fiber trajectories are extracted from the DTI and visualized using 2D colorcoded fiber orientation maps. These maps provide unique information, which cannot be obtained with other MRI techniques. In particular, the fiber orientations could classify and stratify specific WM tracts in a host of medical applications that need more anatomical details [139].

These 2D color-coded maps present the voxel-wise fiber orientations related to the WM tracts, but cannot reveal 3D WM trajectories and connection patterns [26]. The latter are obtained with the computer-aided 3D WM fiber tractography, recognizing and tracing WM tracts and their connections with other WM tracts or GM structures. The tractography is either deterministic, or probabilistic $[32,146]$, and constructs, respectively, only one trajectory for each start voxel, or the most probable, or minimum-energy path between two selected voxels or regions [26]. 

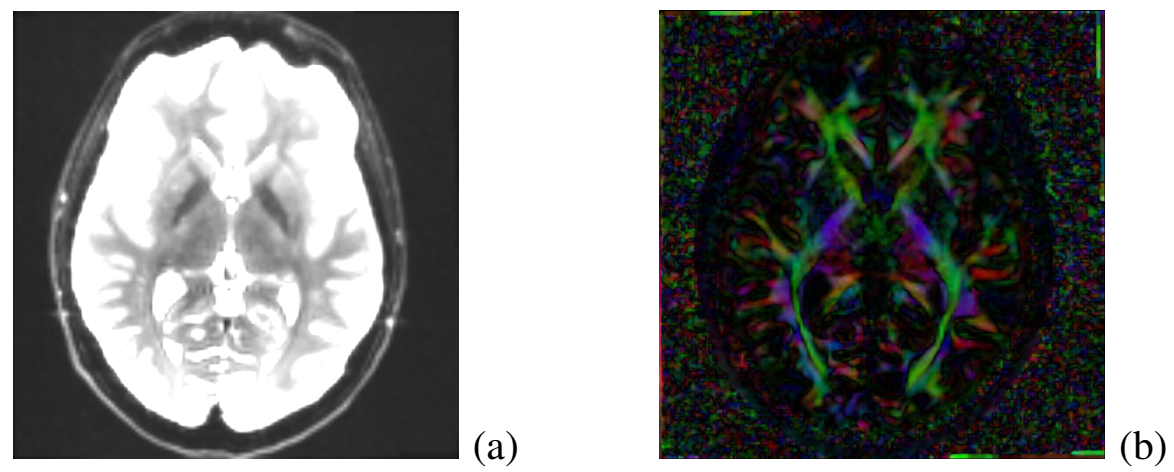

FIGURE 7: Reference non-diffusion image (a) and the color-coded fiber orientation map (red, green, and blue correspond to the diffusion $x-, y$-, and $z$-axes respectively).

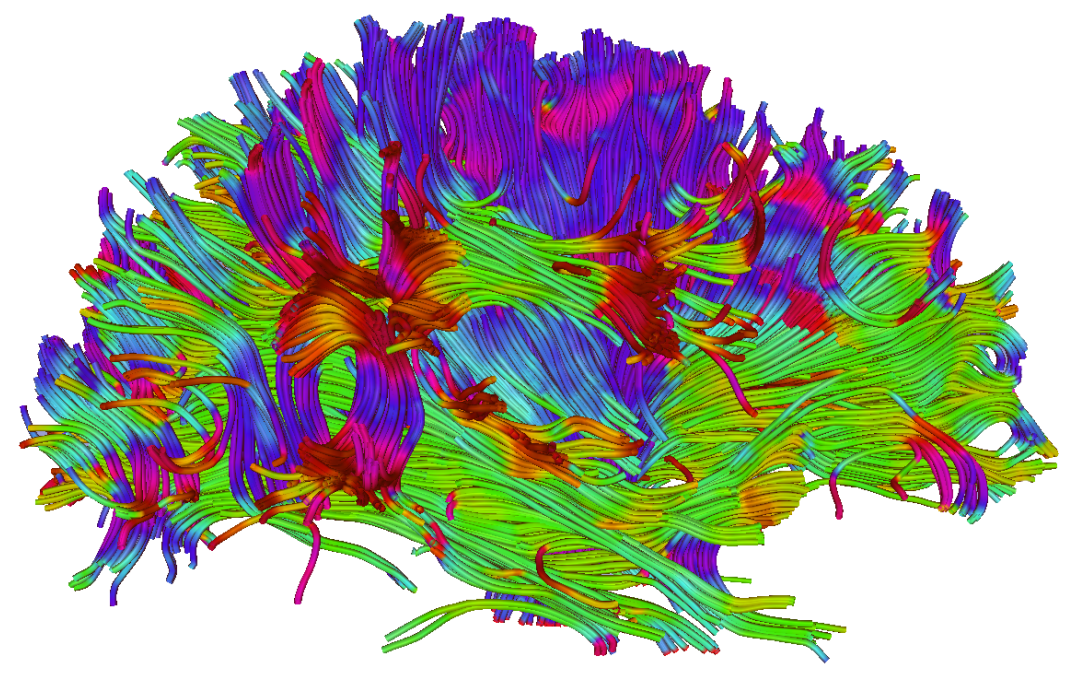

FIGURE 8: Full brain tractography using the "3D Slicer" software: color-coded main fiber directions.

The deterministic (or tract propagation) fiber tractography is built by extracting fiber orientation and propagating pathway until termination criteria are met [26]. Generally, the local voxel-wise fiber orientations are estimated directly from planar diffusion profiles. These estimates fail if the ellipsoid is isotropic or the diffusion profile is planar.

Image noise, patient movements, and other imaging artifacts cause uncertainty in the fiber orientations obtained by the deterministic fiber tractography. The probabilistic fiber tractography attempts to increase the confidence [147] by estimating probability 


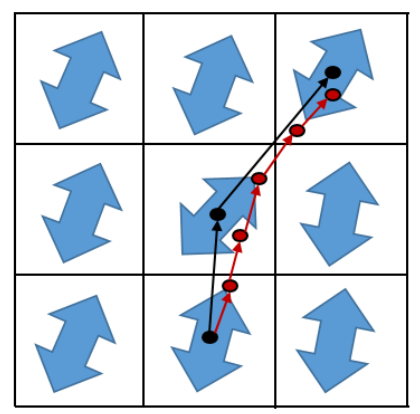

(a)

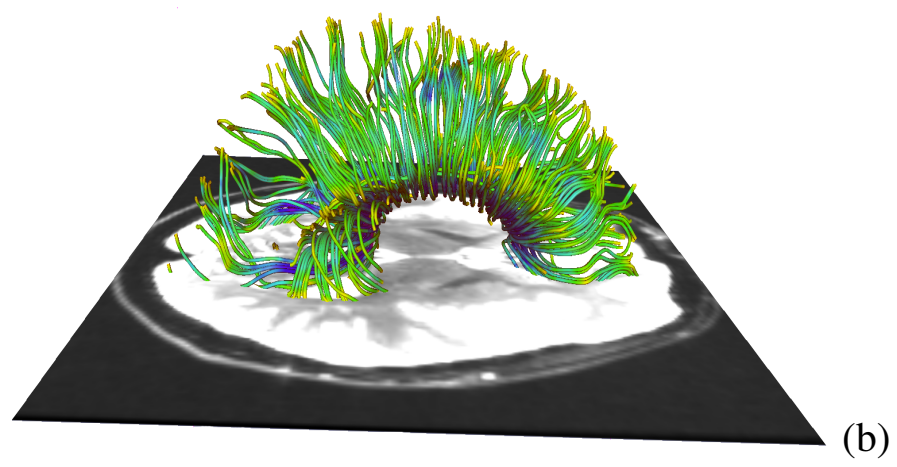

FIGURE 9: Discrete (black) vs. smoothed continuous (red) tracking (a) and deterministic fiber tracts generated with the "3D Slicer" software in the corpus callosum ROI (colors represent the FA values along the tracts).

distributions of all fiber orientations and selecting the most probable orientations. Tracing many different pathways with marginally altered orientations allows for measuring the connection probability and assessing fiber connectivity between different brain regions by a voxel-wise connectivity index [148]. The main advantage of the probabilistic fiber tractography is its ability to stratify the entire WM tracts. However, its accuracy is limited and depends on the accuracy of the DT and estimated pathway probability distributions. Moreover, the probabilistic tractography cannot differentiate between ante- and retrograde along the fiber's path [147].

2. Studying ASD impacts on anatomical structures with DTI

Recent molecular and functional ASD studies confirmed the vital importance of localizing atypical development and examining neural networks and connectivity of different brain areas [149]. At the molecular level, the postmortem studies of brains of children with ASD have shown more reduced and less compact minicolumns [12,150,151]. At the functional level, examining brain connectivity with the fMRI revealed how activities of various brain areas are organized. Most of the ASD patients have demonstrated reduced connectivity between the frontal and posterior brain parts during various cognitive tasks and in a 
resting state [152]. Therefore, according to both molecular and functional studies, the brain connectivity and the underlying WM tracts might be impaired in these patients.

However, earlier studies had limited abilities to provide sufficient morphological information about these WM tracts and their development in a living human [149]. To overcome this drawback, multiple noninvasive DTI-based studies of both the macro- and microstructure (e.g., axons) of the brain WM tracts were conducted for the last decade to investigate the ASD [149]. In particular, the DTI facilitated examining microstructural properties of the WM circuitry and detecting abnormalities of the WM fiber tract integrity [153]. Below, the most important current methods of studying the ASD with the DTI (in total, from 60 publications) are summarized by stratification into five categories: $(i)$ the wholebrain voxel-based analysis (VBA); (ii) the analysis of tract-based spatial statistics (TBSS); (iii) the ROI analysis; (iv) tractography; and (iv) the classification-based analysis. Since the DTI findings in the literature are mainly concerned with WM connectivity across multiple structures, classification here is not based on the structures, such as in Section I.C. It is rather based on the methodology the WM connectivity and the fiber tracts through those structures are investigated with. These methods differ in how group differences are investigated by measuring DTI heterogeneity, e.g., the FA. The same four age stages, as in Section I.C, i.e., infancy, childhood, adolescence, and adulthood, are considered separately for more in-depth presentation of the ASD findings.

a. Whole-brain VBA The brain images across subjects are spatially co-aligned in order to ensure that each individual voxel has the same anatomic location in all the subjects. Then the voxel-wise statistics are used to find areas of significant difference between the ASD and control patients. Due to its comprehensive examination, the wholebrain VBA overcomes problems of possible user bias in and insufficient prior knowledge for selecting the ROI to be analyzed. However, the VBA has drawbacks that are absent in the ROI analysis: high sensitivity to accuracy of aligning the images and low reliability of voxel-wise statistical decisions. In spite of these drawbacks, the VBA is still widely used 
due to its simplicity and ability to explore the whole brain.

Childhood Relationships between communication, social interaction, and repetitive behaviourial impairments have been investigated in [154] by the VBA of FA data for each group of subjects. For the ASD group, the bilateral prefrontal and temporal regions had reduced FA values, as well as lower FA along the frontal striatio-temporal pathways or more posterior brain pathways were associated with communication and social reciprocity impairments or repetitive behaviours, respectively. In a multi-modality study [155], the WM abnormalities were investigated in HFA Chinese children, and the VBA showed that the WM density decreases in the right frontal lobe, left parietal lobe and right anterior cingulate. In addition, the FA values were lower in the frontal lobe and left temporal lobe. Combining the VBA and RBV of the DTI has showed consistent WM abnormalities in the HFA patients. Children with ASD and their unaffected siblings have been compared with the control group in [156]. The ASD and sibling groups had the prevalent reduced FA and $\lambda_{\|}$values in the frontal, parietal, and temporal lobes, especially, in the regions related to social cognition. However, no significant relationships between the WM measurements in the ASD and sibling groups have been reported. To what extent disconnectivity of networks, which are important for social communication, relates to behavioral impairments in children with ASD was investigated in [157]. The VBA indicated the decreased FA values in the uncinate fasciculus and right superior longitudinal fasciculus. The additional analysis revealed a negative correlation between the FA values of the affected fiber tracts and the ASD symptoms.

Adolescence An early study [158] of the WM abnormalities performed the VBA on a small group of male HFA children and adolescents. It has found low FA values in various brain regions, mainly, in the WM adjacent to the ventromedial prefrontal cortices, in the anterior cingulate gyri, and in the temporoparietal junctions. A similar, but larger study [159], has investigated the WM abnormalities in a large male HFA group aged from 
10 to 35 years. The HFA subjects had lower FA values near the corpus callosum and in the right retrolenticular portion of the internal capsule. A new tissue-specific, smoothingcompensated (T-SPOON) VBA introduced in [160] minimizes effects of partial volume averaging and image smoothing by applying a regional mask with the same smoothing parameters. Compared to the conventional VBA, results of the T-SPOON on a large group of the ASD subjects and corresponding controls were more consistent with the FA obtained by analyzing the corpus callosum and temporal lobe ROIs. The VBA of the WM of a small group of HFA and matched controls in [161] has shown the reduced FA and $\lambda_{\perp}$ in various ROIs in the brain, including the left dorsolateral prefrontal cortex, cingulum bundle, arcuate fasciculus, and superior longitudinal fasciculus. Social impairment scores correlated negatively with the FA of the left dorsolateral prefrontal cortex, suggesting that the WM in cortical regions is vital for the ASD patients development. The WM integrity of the major fiber tracts connecting the amygdala, fusiform face area, and superior temporal sulcus in the ASD subjects was explored in [162] by the FA-based VBA. The ASD subjects had reduced FA values in the investigated WM tracts, including inferior longitudinal fasciculus, inferior fronto-occipital fasciculus, superior longitudinal fasciculus, corpus callosum, and cingulum bundle. A multi-modality study [163] used the T1 MRI to study the brain volumetrics and the DTI to investigate both the WM and GM integrity in a limited group of HFA adolescents. There were no significant volumetric GM or WM differences between the two groups.

Adulthood The ASP adults, examined in [164] for the WM integrity of the whole brain, have shown lower FA of 13 WM clusters in the internal capsule, frontal, temporal, parietal, and occipital lobes, as well as the cingulum and corpus callosum. Studying the WM abnormalities on a small group of young LFA men [165] indicated a positive correlation between the FA in the uncinate fasciculus and clinical improvement, precocity, and intervention duration. 
b. Tract-based spatial statistics (TBSS) To overcome some shortcomings of the traditional VBA, the recent DTI-optimized analysis of TBSS [166] uses a non-linear registration to a common target to co-align the subjects' FA maps. Following the alignment, a mean FA skeleton is built and thresholded to exclude areas of high inter-subject variability. Based on the corresponding FA values, each aligned subject's FA map is projected onto the skeleton to facilitate collecting standard voxel-wise FA statistics across all the subjects. Generally, such tract-based analysis is more accurate than the VBA, but requires a sophisticated data projection method for better results. It also does not handle partial volume effects, is sensitive to motion distortions, has high computational complexity, and may fail if the tracks change much at junctions or due to apparent pathologies.

Childhood The deterministic tractography and TBSS were combined in [167] to investigate the corpus callosum region, including the uncinate fasciculus, in young ASD children aged five years on average. The ASD manifests itself in lower FA, higher MD, larger number of streamlines and voxels, and longer streamlines. There were also ASD-related macrostructural changes in the uncinate fasciculus correlate with the popular symptomatic scores of the GARS (Gilliam autism rating scale). The TBSS, VOI, and tractography have been used also in [168] to investigate the WM abnormalities of very young ASD children in several clusters within the genu and body of the corpus callosum, left superior longitudinal fasciculus, and right and left cingulum. The FA increased in these regions as a consequence of the decreased radial diffusivity, $\lambda_{\perp}$. The tractography revealed that increased FA was concentrated in the mid-body of the corpus callosum and in the left cingulum.

To study the integrity of the thalamic radiation of older ASD children in [169], four DTI measurements, namely, FA, MD, $\lambda_{\perp}$, and $\lambda_{\|}$, were examined in the anterior thalamic radiation, superior thalamic radiation, posterior thalamic radiation, corpus callosum, uncinate fasciculus, and inferior longitudinal fasciculus by combining the whole brain VBA analysis with the TBSS and ROI analyses. Anticipated WM abnormalities in 
the thalamo-frontal connections in the ASD children are indicated by the reduced FA and $\lambda_{\|}$and Increased MD and $\lambda_{\perp}$ across various brain regions. The TBSS, combined with a VBA were applied in [170] to a small group of older ASD and control children. The FA was reduced, especially, in the forceps minor, inferior fronto-occipital fasciculus, and superior longitudinal fasciculus. Regional distributions of differences between young ASD and control children were examined in [171] using the TBSS and the whole-brain VBA. While the FA values were reduced in various brain regions, the increased MD was found only in the posterior ones. These small (1-2\%) regional differences between both groups were accompanied by distinct regional differences in imaging artifacts. They also demonstrated vulnerability of the between-group differences to such artifacts, which may cause errors in biological inferences. The TBSS and deterministic tractography were also used in [172] to analyze young ASD children with and without mental retardation. The statistics have detected a widespread FA increase in major WM pathways, and the tractography showed increased FA and fiber length in the cingulum and corpus callosum. Moreover, the MD increase was correlated with expressive language functioning in the indirect segments of the right arcuate and left cingulum.

Adolescence Examining differences in the WM integrity between the HFA and control subjects and its association with pictorial reasoning under various linguistic levels in [173] indicated that visuospatial reasoning performance relates to the FA of the peripheral parietal and superior precentral WM in the HFA subjects, but the superior longitudinal fasciculus, callosal, and frontal WM in the controls. The whole-brain VBA followed by analyzing the TBSS in [174] evaluated the WM in a group of ASD and control children and adolescents. The VBA has shown the increased MD and radial diffusivity $\left(\lambda_{\perp}\right)$ in the ASD children, but not the adolescents in the frontal WM, and the statistics analysis has revealed alterations in the right uncinate fasciculus. The right inferior longitudinal fasciculus in the ASD case may indicate a disrupted fronto-temporal-occipital circuit playing a significant role in social 
and emotional processing. The TBSS was analyzed in [175] to examine short- and longdistance WM tracts in the frontal, parietal, and temporal lobes of the ASD subjects and matched controls. The short-distance tracts had reduced FA in the ASD group, increased MD and $\lambda_{\perp}$ in the frontal, temporal, and parietal lobes. The age and DTI measurements were correlated in the control, but not ASD group. As was suggested, these typical agerelated correlations were absent due to altered maturation of the short-distance tracts in the ASD group.

The whole-brain VBA with TBSS in [176] assessed the WM tracts in the ASD children and adolescents. The reduced FA and the higher MD and radial diffusivity have been found for the ASD subjects in the corpus callosum, anterior and posterior limbs of the internal capsule, inferior longitudinal fasciculus, inferior fronto-occipital fasciculus, superior longitudinal fasciculus, cingulum, anterior thalamic radiation, and corticospinal tract. Moreover, the age-dependent analysis has shown no maturational changes in the ASD subjects. The WM tracts of a relatively larger group of the adolescents were studied in [177] by examining the TBSS after correcting the entire image, rather than a ROI, which is more typical. As was found in the ASD group, the FA has increased, specially in the right inferior fronto-occipital fasciculus and affected visual perception. It suggests an abnormal information flow between the insular salience processing areas and occipital visual areas of the ASD patients.

Adulthood A recent fMRI and DTI study [178] examined causal attribution in the ASD and confirmed the relationship for the temporo-parietal junction that exists in the theory of mind. The response to intentional causality showed lower activation of the temporoparietal junction in the fMRI of the ASD adults, and the analysis of the tract-based spatial DTI statistics revealed reduced FA in the temporal lobe. The tract-based statistics were used also in a more recent investigation [179] of the WM integrity in a small group of the ASD adults and their matched controls. The ASD subjects showed significantly decreased 
FA and high radial diffusivity, $\lambda_{\perp}$, values in the left hemisphere, mainly, in the thalamic and fronto-parietal pathways. Moreover, the WM disturbance was higher in the left hemisphere.

c. ROI analysis These analyses depend on prior assumptions about certain brain regions that might be impaired in the ASD subjects. The regions are extracted manually, semi-automatically (using delineation protocols), or automatically. Manual extraction is time-consuming and suffers from user variability, whereas automatic and semi-automatic region segmentation are affected by registration errors, as in the VBA. The fact that the VOI are grouped in a predefined way is the main advantage of analyzing the ROI, as the total number of multiple comparisons is much smaller than in the VBA, statistical decisions become more accurate. However, the ROI analysis cannot infer conclusions about microstructural properties of the WM tracts. It is impractical for examining every brain region, especially for large groups, and has limited accuracy because of low resolution and relatively thick slices of the DTI.

Infancy Microstructural WM differentiation between the ASD and control groups of infants and very young children was examined in [180]. The fact that the left hemisphere's frontal lobe of the ASD subjects had predominately increased FA and probability, as well as reduced displacement supports the previous findings indicating an abnormal brain overgrowth in very young children.

Childhood The reduced FA of various cerebral WM tracts, which was found in [181], suggests the ASD correlates with reduced connectivity in the corpus callosum, internal capsule, and superior and middle cerebellar peduncles. The ROI analysis of the cerebellar outflow and inflow pathways in [182] has shown the bilaterally decreased MD in the superior cerebellar peduncles, together with the asymmetric FA of the middle cerebellar peduncle and the inferior cerebellar peduncle in the ASD patients. The effect of selfinjurious behavior on cortical development of the ASD children was studied in [183] by using both T1 MRI and DTI scans. According to the sMRI analysis, both the thickness 
of the right superior parietal lobule and bilateral primary somatosensory cortices and the volume of the left ventroposterior nucleus of the thalamus correlate with the self-injury scores. The atlas-based ROI analysis has revealed that children engaged in self-injury had significantly decreased FA and increased MD in the the left posterior limb of the internal capsule, as well as increased radial diffusivity, $\lambda_{\perp}$, in the bilateral posterior limbs of the internal capsule and corona radiate. Recently, an atlas-based ROI analysis [184] was used to investigate abnormalities in various left and right hemispheric WM regions of the HFA children. Their significantly increased MD of the outer-zone cortical left hemisphere WM suggested hypomyelination and increased short-range cortico-cortical connections caused by the early WM overgrowth.

Adolescence The entire corpus callosum and its subregions (genu, body and splenium) were explored for a large group of the HFA subjects and matched controls in [139] using the DTI (FA, MD, $\lambda_{\perp}$, and $\lambda_{\perp}$ ) and RBV. The low-IQ HFA subgroup differed by small corpus callosum volumes, increased MD, decreased FA, and increased $\lambda_{\perp}$. The decreased FA and increased MD and $\lambda_{\perp}$ in the superior temporal gyrus and temporal stem in the HFA subjects of the same group have been reported in [185]. Analyzing the WM over the whole brain and in several ROIs in [186] has detected decreased FA and increased $\lambda_{\perp}$ in both the whole brain and corpus callosum; increased MD for the whole brain and the anterior and posterior limbs of the internal capsule; decreased $\lambda_{\|}$in the corpus callossum body, and decreased FA in the middle cerebellar peduncle of the HFA subjects.

Adulthood The fMRI and DTI have been used in [187] to determine whether the structure and function of the anterior cingulate cortex relate to a repetitive behavior in the ASD. The ASD subjects showed an increased rostral anterior cingulate cortex activation to both correct and erroneous responses and had reduced FA in the WM underlying the anterior cingulate cortex. These results correlate also with ratings of the rigid, repetitive behavior. A multi-modality study [188] used the VBM and ROI analysis to estimate the GM and WM 
volumes from the sMRI and evaluate the main WM tracts in the DTI, respectively, for the ASP adults. The total WM volume, regional GM volume in the right parietal operculum, and FA in the body of the corpus callosum, cingulum, and cerebellum suggested a correlation between the diagnosis and subject's gender. These findings confirmed the importance of understanding the sex-specific brain differentiation in the ASD.

d. Tractography Tractography reconstructs virtual 3D trajectories of the WM tracts to define the ROIs required for examining several such tracts simultaneously and characterizes macrostructural tract properties with additional DTI measurements. The deterministic tractography reconstructs the WM tracts and measures their lengths, densities, or volumes from a number of streamlines propagated between voxels with similar diffusion properties. But it provides no uncertainty of the reconstructed tracks due to noise or insufficient spatial resolution, whereas the probabilistic tractography not only estimates the fiber tracts, but also measures their uncertainty. However, branching and false-positive tracts affect the tractography accuracy in defining the ROIs, specifically at the ends of the reconstructed tracts. Also, the DTI tractography fails if the DT is inadequate to describe a region. For example, the FA is significantly lower for complex WM fiber crossings. To meet these challenges, new diffusion models, scanning paradigms, and analysis methods are constantly being developed.

Infancy Data from the IBIS (infant brain imaging study) group have been used for a recent longitudinal DTI analysis [153] of how the WM fiber tract is organized from 6 to 24 months in high-risk siblings' infants, who developed the ASD by 24 months. The FA and axial $\left(\lambda_{\|}\right)$and radial $\left(\lambda_{\perp}\right)$ diffusivity measured for the DTI were investigated to characterize microstructural properties of the WM fiber tracts. The FA for the infants who developed the ASD differed significantly from those who did not develop it in 12 out of the 15 fiber tracts investigated, including the fornix, uncinate fasciculus, and inferior longitudinal fasciculus. For most of the investigated ASD infants, the FA of the developing fiber tracts was higher at 
6 months, had no differences at 12 months, and decreased at 24 months of age. This study provided evidence for the altered brain growth of the WM pathways related to manifesting autistic symptoms for the first year of life, thus confirming the critical importance of the longitudinal studies in revealing the age-related brain and behavior changes underlying the neurodevelopmental disorders. The DTI tractography in [189] used the same IBIS group, but focused on determining whether specific oculomotor functioning and visual orienting patterns characterize 7-month-old infants diagnosed with the ASD at 24 months and detecting neural associates of their behaviors. The measurements included an average saccadic reaction time in a visually guided saccade procedure and the radial diffusivity, $\lambda_{\perp}$, of corticospinal pathways and the splenium and genu of the corpus callosum fiber tracts. Strong association between the $\lambda_{\perp}$ of the splenium of the corpus callosum and visual orienting latencies in low-risk infants has been found, but this correlation was missing in the infants having the ASD. These results confirm the potential of acquiring infant imaging groups in identifying early ASD markers, which is critical for early clinical intervention.

Childhood Whether the deterministic tractography of the DTI can detect the WM abnormalities in the frontal lobe and check for short range connectivity changes in the ASD children was investigated in [190]. The higher MD in the whole frontal lobe, as well as in long and short range association fibers in the ASD group have been reported. The FA was reduced in the ASD group for the short range, but not long range fibers, and the necessity of advanced DTI technology to re-examine the short range connectivity in ASD was indicated. The brain connectivity in the corpus callosum of the HFA patients was examined in [191] by measuring both the DTI and T1 MRI. The corpus callosum volume and density extracted from the MRI were compared to the FA, MD, average fiber length, and fiber number obtained from the DTI by deterministic tractography. The decreased WM density in the anterior third of the corpus callosum, and the higher MD and lower fiber number in the anterior third transcallosal fiber tracts of the HFA subjects have been found. The 
frontal lobe association pathways in the ASD children were studied in [192] by analyzing the tract curvature, FA, $\lambda_{\|}$, and $\lambda_{\perp}$. This study suggested that higher curvatures and $\lambda_{\perp}$ in the parietotemporal junction of accurate fasciculus, frontotemporal junction of uncinate fasciculus, and the midline of the genu of the corpus callosum could be caused by larger attenuation of the thinner axons in the frontal lobe tracts of the ASD children.

The lack of the nonverbal ASD studies was addressed in [193] by employing the probabilistic tractography to find language-related WM tracts (arcuate fasciculus). A small group of five nonverbal ASD children demonstrated the reversed arcuate fasciculus asymmetry. To what extent the ASD language ability is associated with the DTI measurements (FA and MD) of the language-related WM tracts (superior longitudinal fasciculus) and non-language-related WM tracts (corticospinal tracts) was investigated in [194] with the deterministic tractography. The obtained results have revealed the higher MD in the left hemisphere temporal portion of the superior longitudinal fasciculus fiber tracts of the ASD children with language impairment, as well as a significant negative correlation between the MD and language ability scores. The fMRI and probabilistic tractography of the DTI in [195] examined the functional and structural organization of neural systems overlapping for language and music in the ASD children. The ASD children have demonstrated decreased functional responses to speech stimulation in the left inferior frontal gyrus and secondary auditory cortices in the left temporal lobe, as well as decreased FA of the left dorsal pathway and the decreased tensor norms in the ventral tract. All the findings indicated that speech and song processing functional systems are more responsive for the song than the speech of the ASD children.

Relations between the WM microstructure and developing the morphosyntax in a spoken narrative were examined in [196] on a small group of the older HFA children and their matched controls. The HFA children showed abnormally increased MD in the right inferior longitudinal fasciculus. A positive correlation between the morphological accuracy and WM integrity in the right inferior longitudinal fasciculus was found within the HFA 
group. This study has shown that the HFA children rely on the ventral, rather than typical pathways in their daily use of real world language. Both the sMRI and probabilistic DTI tractography were used in [197] to investigate the GM and WM related to language ability in the young ASD children. Compared to the control group, the ASD children had decreased leftward asymmetry of volume and $\lambda_{\perp}$ of the arcuate fasciculus, but no difference in the GM asymmetries.

Adolescence Examining the arcuate fasciculus in a group of ASD and control adolescent subjects with the probabilistic tractography [198] showed no group differences in FA. But atypical language laterality was more predominant in the ASD, than the control group. Advantages of volumetric DTI segmentation over tractography (its higher robustness to imaging noise, better compatibility with statistical analysis, and no region-to-region analysis) were exemplified in [199] on extracting the WM tracts to study the same arcuate fasciculus on a group of the adolescent HFA and control subjects. The ASD group had increased MD and $\lambda_{\perp}$, and decreased FA. The overlap between specific LI and ASD LI in a group of the adolescents and children has been studied in [200]. The deterministic tractography was applied to extract the superior longitudinal fascicle, and no significant differences in the FA and MD have been revealed between the ASD LI participants and controls. However, the FA was significantly reduced in children with the specific LI compared to their controls. The diffusion tractography was used in [201] to investigate three association fibers, such as the bilateral cingulum bundle, bilateral arcuate fasciculus, and bilateral uncinate fasciculus, as well as the callosal fiber tracts. High-resolution diffusion spectrum imaging (DSI) was applied to a small group of the HFA subjects and controls in order to assess generalized fractional anisotropy (FA) and asymmetry patterns in the targeted fiber tracts. The HFA adolescents showed no leftward asymmetry found in the controls, but had the significantly reduced generalized FA in the three callosal fibers. The deterministic tractography in [202] extracted the cingulum bundle from a larger group of children and adolescent HFA sub- 
jects and controls. Significant age group interaction for the FA and diffusivity (MD, $\lambda_{\perp}$, and $\lambda_{\|}$), which is driven by reduced FA and increased diffusivity in the ASD groups, but not in the adolescent groups, has been revealed. The fMRI and DTI probabilistic tractography were used in [203] to examine the integrity of thalamo-cortical connectivity in children and adolescents with the ASD. Increased MD and $\lambda_{\perp}$ in the thalamo-cortical connections, and decreased functional connectivity in the thalamo-cortical circuitry, as well as a negative correlation between the fronto-thalamic FA and the social and total autism diagnostic observation schedule (ADOS) scores were reported. The cingulum bundle of a group of the ASD and control adolescents and young adults was investigated in [204]. By applying the probabilistic tractography, the low FA of the bilateral anterior cingulum bundle and negative correlation between this FA and the behavior rating inventory of executive function (BRIEF) scores were also identified.

Adulthood The deterministic tractography was used in [205] to examine microstructural integrity of the intracerebellar pathways in the ASP adults. The study revealed reduced FA of the short intracerebellar fibers and right superior cerebellar output peduncle. It also showed negative correlation with the autism diagnostic interview (ADI) scores. The deterministic tractography in [206] reconstructed the WM tracts from the amygdala to the fusiform cortex, and the hippocampus to the fusiform cortex in the ASD and control adults. The ASD group has shown increased $\lambda_{\perp}$ in the bilateral amygdala fusiform connections and left hemisphere hippocampus fusiform connections, as well as decreased $\lambda_{\perp}$ in the right hippocampus-to-fusiform connections, being associated with the lower face recognition scores and performance IQ. The very first study [207], which examined connections between socio-emotional structures in the adults with ASD using the deterministic tractography, reported an increased number of streamlines (tract volume) in the right cingulum bundle and inferior longitudinal fasciculus of the ASP adults in contrast to their reduced number of streamlines in the right uncinated fasciculus. Significant age-related differences 
between the autistic and control groups were found in the MD of the left uncinate fasciculus. The deterministic tractography was used also in [208] to extract the intra-hemispheric visual-association WM tracts for the HFA adults and detect their high numbers of streamlines in the intra-hemispheric fibers, mainly, in the left hemisphere, and a low number of streamlines in the minor forceps and the body of the corpus callossum. A multi-modality (sMRI and DTI) approach in [209] has examined differences in the bulk striatum volume and fronto-striatal WM integrity and their relationship with repetitive behavior and inhibitory control of the ASD adults. Analyzing the MRI has shown a smaller WM volume, and the DTI tractography revealed reduced FA in the WM connecting putamen to the frontal cortical areas and increased MD of the WM tracts connecting the accumbens to the frontal cortex. The relationship between the inter-hemispheric connectivity and the brain overgrowth in the ASD adults has been tested in [210] with the probabilistic tractography. The estimated callosal fiber length measured the maximum brain size achieved during the development, and compared to the size and structure of the corpus callosum extracted from the T1-weighted MRI. In the ASD adults, the callosal fiber length correlated inversely with the corpus callosum size and positively with the radial diffusivity, $\lambda_{\perp}$.

e. Classification-based analysis Since the advent of their basic concept in the mid-1980s, computer-aided diagnostic (CAD) systems remain in great demand among neuroradiologists [211]. Most of the present CAD systems perform data preprocessing, feature extraction, and classification. Initial efforts to use features extracted from DTI to classify and diagnose ASD are detailed below.

Childhood Different DT coefficients from many areas across the brain were used in [212] to distinguish autistic children from controls. A high-dimensional nonlinear SVM has learned an underlying ASD pattern of numerous atlas-based regional DTI features, e.g., FA and MD, extracted from the various brain ROIs. According to the leave-one-out (LOO) cross validation, $84 \%$ specificity and $74 \%$ sensitivity were achieved in separating the autis- 
tic patients from the control ones.

Adolescence Shape representations of the WM tracts extracted from the DTI were utilized in [213] to distinguish between the autistic and control patients. These fiber bundles were seeded in the splenium of the corpus callosum, and the classification features were built using 3D shape context [214]. The LOO cross-validation has resulted in an accuracy of $75 \%$; both the specificity and sensitivity of $71 \%$, and an average AUC of 0.765 (the area under the receiver operating characteristic curve). The DTI features of the ROI from the WM of superior temporal gyrus and temporal stem brain regions, which were assumed to have an important role in language, emotion, and social cognition development, were used in [215] to study a large group of the HFA subjects and their matched controls. The extracted features included the FA, MD, $\lambda_{\perp}, \lambda_{\|}$, normalized DT skewness, and hemispheric asymmetry index. Using the selected aforementioned six WM measurements, the system was able to separate the ASD subjects from the controls with $92 \%$ accuracy; $94 \%$ sensitivity, and $90 \%$ specificity.

\section{E. Studying ASD with fMRI}

As mentioned earlier, fMRI studies attempt to focus on brain activity in autism by detecting changes associated with blood flow, Figure 10. The fMRI studies can be categorized based on the different task domains [27,28]. Examples include motor tasks, visual processing tasks, executive function tasks, auditory and language tasks, basic social processing tasks, and complex social cognition tasks.

Motor tasks experiments in the literature [216-221] revealed that the bilateral precentral gyri and the inferior frontal gyri are more activated in cases with autism than they are in neurotypicals. On the other hand, the left culmen and the right superior temporal gyrus showed greater activation in neurotypicals. Allen et al. performed experiments in order to examine cerebellar activity $[216,217]$, where eight autistic patients and their matched 


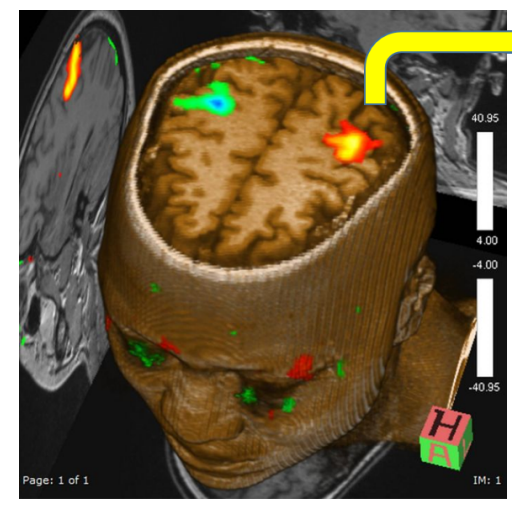

Areas of Activation

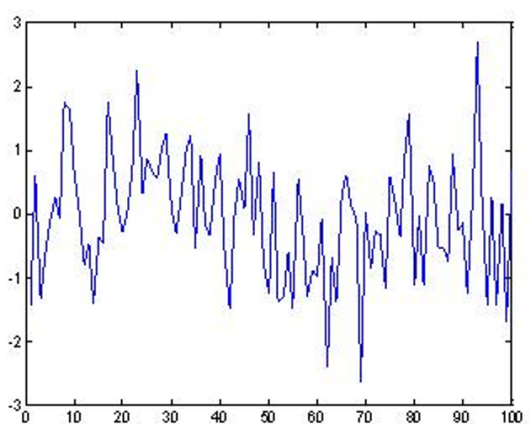

BOLD Signal

FIGURE 10: Localizing BOLD effects and correlating them to areas of activation in the brain.

controls aged between 14 and 38 years performed both motor and attention tasks. A button was pressed and activation was compared with a rest condition for the motor task. For attention, visual stimuli were presented one at a time at fixation and subjects pressed a button to every target. Anatomic ROIs in the cerebellar cortex were then manually identified guided by MRI atlases of the human cerebellum. Results showed high motor activation in autistic subjects in the ipsilateral anterior cerebellar hemisphere compared to normal subjects. Muller et al. [218] tested eight autistic adult males and their matched controls for motor activity during sequence learning. After data preprocessing and normalization, the hemodynamic effects associated with task-control cycles were statistically examined using the general linear model (GLM). Results showed the less prefrontal activation in autism along with the enhanced activation in the right pericentral and premotor cortex compared to neurotypicals. The study in [221] conducted a comparison between ASD and control young adults, which recorded activation due to a simple hand motion task. The BOLD signal activation was modeled using GLM and analyzed using SPM package. Both groups had same areas activated as prefrontal, inferior parietal and superior temporal regions, but ASD showed higher activation in the dorsal premotor cortex and less activation in the cuneus and the middle temporal gyrus. 
Visual processing tasks are the sequential steps needed all the way from the visual sensors to the cognitive processing in order to process information. FMRI studies in the literature found that the thalamus and the medial frontal gyrus showed more activation in autism, whereas the cingulate gyrus and the occipital region showed more activation in neurotypicals [187, 222-228]. Manjaly et al. [224] examined 12 autistic adolescents and their matched controls for visuospatial tasks, particularly, the Embedded Figures Task (EFT). SPM tool was used to analyze the data using GLM, where specific effects were tested for by applying linear contrasts to the estimated parameters in order to obtain contrast images. Group analyses were then performed on the contrast images. Results showed the overactivation in the right primary visual cortex and the bilateral extrastriate areas of autistic patients. Takarae et al. [227] performed a visual task experiment using saccadic and pursuit eye movement on 13 high-functioning autistic individuals and 14 matched controls. The study used FIASCO package to correct for motion artifacts. F-statistic maps were then created in order to identify significant differences between groups for each task. The maps were calculated by dividing voxel-wise chi-square values form within-group activation maps by the corresponding degrees of freedom that are appropriate for each group. Autistic individuals showed greater activation in the dorsolateral prefrontal cortex, caudate nucleus, medial thalamus, and anterior and posterior cingulate cortex. Clery et al. [228] used fMRI to study the impact of unexpected visual changes on both adult ASD and typically developing groups, where the variation in BOLD signal was modeled using GLM, and then analyzed using SPM package. In both groups, changes were detected in the occipital and frontal regions, while autistic subjects exhibited more changes in the bilateral occipital cortex.

Executive functions, also known as cognitive control functions, are a group of cognitive processes that are responsible for many activities, including problem solving and reasoning. Areas of activation for executive function tasks were found to be in the left middle frontal gyrus in autism, and in the right middle frontal gyrus and prefrontal and subcortical 
regions in neurotypicals [229-236]. In order to characterize the cognitive deficits in ASD, Dichter et al. [229] conducted an event-related experiment in order to examine the effects of processing directional information from faces on activity within brain regions mediating cognitive control. Preprocessing of scans was conducted using SPM modules, followed by random-effects analysis of the differences between conditions, e.g., incongruent vs. congruent gaze stimuli w.r.t. hemodynamic responses (HDRs). t-statistic maps were then computed at each voxel in order to quantify HDR differences between conditions. Autistic individuals showed hypoactivation with the incongruent gaze stimuli in the bilateral midfrontal gyrus, bilateral intraparietal sulcus, and in the anterior cingulate. Gilbert et al. [230] performed two executive function tests on 15 high-functioning autistic individuals and 18 matched controls. Data were analyzed using SPM2 package in a GLM that decomposes the BOLD signal variance with a set of regressors. There were no significant differences on the behavioral level. Also, executive function classical test showed differences between the two groups in only the cerebellum. However, a new test of executive functions showed significantly greater BOLD signal change in the medial rostral prefrontal cortex in autism as well as abnormal functional organization of medial prefrontal cortex. Koshino et al. [233] investigated brain activation of autistic individuals using a working memory task that involved verbal processing, working memory maintenance, and theory of mind processing. Data were analyzed using SPM99 package, where maps were calculated for contrasts between the experimental conditions and fixation and between the two groups. Individuals with autism showed lower activation in the inferior left prefrontal and the right posterior temporal areas than the control group. Lee et al. [234] examined cognitive skills for 12 autistic children and their matched controls by investigating the connectivity of the left and right inferior frontal cortex and of other regions in the frontal, striatal, and parietal cortex. Group analyses were conducted in SPM5, where fMRI responses were modeled by a canonical HRF and activation maps for each subject were generated using a linear contrast.

As per the auditory and language tasks, studies conducted on children and ado- 
lescents in the literature showed greater activation in autism in the right precentral gyrus region, whereas the bilateral superior temporal gyri were more activated in neurotypicals [237-243]. In autistic adults, the bilateral declive was more activated than it is in neurotypicals [244-250]. In all age groups, neurotypicals exhibited greater activation in the left cingulate gyrus. Gomot et al. [238] investigated extreme repetitive behavior (resistance to change) in 12 autistic children and their matched controls at sensory and neural levels in order to examine auditory novelty detection and its neural basis. SPM2 package was used to analyze the data and the BOLD signal was statistically analyzed using GLM by separate modelling of the events using a conical HRF and its first-order temporal derivative. The right prefrontal-premotor and the left inferior parietal regions were more activated in autistic individuals than in controls. Knaus et al. [239] conducted a study to examine semantic functions in 12 autistic adolescents and 12 controls. Analyses were done using Neurolens package. After applying motion correction, GLM was performed in order to generate activation maps that would fit the task block's time vector convolved with a gamma variate estimate of HDR. Autistic boys showed stronger activation in Broca's area. Redcay et al. [240] conducted an experiment on 12 autistic children and two control groups in order to identify brain regions involved in speech perception. Analyses were performed using AFNI software package, and GLM was applied to fit the time series events to an ideal HRF. Individuals with autism exhibited greater activation within the right and medial frontal regions, which shows that there is a positive relationship between right hemisphere frontal and temporal activity to forward speech and language skill. Lombardo et al. [243] used fMRI to compare language sensitive superior temporal cortices activity between ASD toddlers of good language outcome, those of relatively lower language outcome, and typically developed toddlers. Data were analyzed using SPM package, and the analysis showed that the activation of the language sensitive superior temporal cortices in both groups of higher language is the same, whereas less activation was detected for those of lower language outcome. In [250], a comparison was held between first degree relatives of both 
ASD and control groups to study their HDRs for phonological processing task, where the BOLD signal activation was modeled using GLM and analyzed using SPM package. The HDRs for parents of ASD were higher than those of the control group.

fMRI basic social processing tasks include face processing [251-254], emotion processing [255-261], and motion in relation to social stimuli [262-266]. Studies in the literature show that greater activation in the bilateral superior temporal gyri is exhibited in autism, whereas neurotypicals exhibit greater activation in the left fusiform gyrus and in the right inferior occipital gyrus. Corbett et al. [252] conducted an fMRI experiment that involved matching facial expressions and people on children with high-functioning autism and their matched controls. Data analyses were conducted sing GLM in SPM2 package. Results showed that children with autism exhibited intact motion matching and showed diminished activation of the fusiform gyrus and the amygdala. Greimel et al. [257] investigated neural mechanisms of empathy in 15 boys with ASD, 11 fathers of adolescents with ASD, and two control groups. The experiment was based on asking the participants about the emotional state they infer from emotional faces shown to them. Analysis was conducted using SPM5, where estimation of the model parameters was conducted using GLM and realignment parameters were included into the model as regressors. Individuals with ASD showed diminished fusiform gyrus activation compared to controls, and also showed less congruent reactions and decreased inferior frontal gyrus activity. Also, fathers of affected individuals showed reduced fusiform gyrus activation when inferring emotions. The study in [261] tried to discriminate between ASD and control adults by exposing both of them to some fearful faces and houses and compare their responses. The data were analyzed using FSL tool and GLM to model BOLD signal, and showed that slower response was exhibited by the autistic group. Dichter et al. [263] examined the effects of presenting highly arousing pictures (both very pleasant and very unpleasant) to individuals with high-functioning autism. Data processing was conducted using SPM modules, and epochal analysis was applied to extract condition-specific activation time courses. The control group showed 
greater activation in the right lateral midfrontal cortex than the autistic group when shown the high-arousal pictures.

Complex social cognition tasks include activities such as imitation, irony comprehension, and empathy. Autistic children and adolescents showed greater activation in the left inferior frontal gyrus, left pre- and post-central gyri and the left superior temporal gyrus than neurotypicals. Reduced activation in autistic children/adolescents was shown in left superior frontal gyrus, right superior temporal gyrus and left inferior parietal lobule [267-270], whereas reduced activation was encountered in the right claustrum with autistic adults [271-273]. Uddin et al. [269] investigated brain response to images of the subjects' own faces and of others on children both with ASD and typically developing. Analysis was conducted using FEAT; part of FSL package, and the BOLD signal was modeled using a separate explanatory variable for each stimulus. Both groups showed activation at the right premotor/prefrontal system when identifying images. However, the control group exhibited this activation when presented both self- and others'- faces, whereas this activation in the autistic group was exhibited mostly with their own faces. Gilbert et al. [271] investigated spatial and verbal tasks on a group of 16 high-functioning adults and their matched controls. Tasks manipulated attention towards perceptual versus selfgenerated information, and reflection on another person's mental state. Data were analyzed using SPM5 package and multi-voxel similarity analyses were conducted, Abnormal functional specialization was disrupted in the autistic group within medial rostral frontal cortex.

\section{F. Limitations of existing work}

The overviewed results of the widespread investigation of the sMRI and DTI modalities in ASD diagnostics outline some limitations of the existing work.

Diagnosing ASD with sMRI Obviously, from the sMRI investigations completed to date, it is clear that the brain of an autistic child is growing abnormally through early 
childhood. However, a consistent pathology amongst the autistic children is yet to be identified, and few, if any, studies include patients below two years of age. Nonetheless, early brain overgrowth does appear to be a consistent and repetitive ASD feature, but this feature is heterogeneous and not all autistic children have an enlarged cerebral volume. Moreover, due to a common misconception of gender bias in the ASD, most of the studies primarily or exclusively focused on males. Also, the statistical significance of the reported findings are hindered due to the small population of subjects considered.

Within the literature, the effects of age on various anatomical features such as, the dynamics of the amygdalae and cerebral volumes, tend to be contradictory. Furthermore, multiple cross-sectional studies examining brain stem size in ASD patients have shown inconsistent results, and no study to date specifically assesses how the brain stem develops with age. Also, studying the brain stem should become volumetric and focus on selecting proper planes of cut, because most of the current studies have transected the brain stem and provided only area measurements. As a result, additional longitudinal studies are needed before any conclusions can be drawn on the role of brainstem development in autism.

Deciphering the role of different brain regions and networks in autism may benefit significantly from using multimodality approaches, which combine various resting state, task-evoked, and structural MRI measurements and rely on advanced data acquisition and analysis.

The utilization of MRI-based methods to study brain development in larger populations will likely begin to address heterogeneity in ASD patients and identify distinct autism subtypes, each with a specific associated neuroanatomical phenotype. However, it is anticipated that the maximum scientific benefit will be achieved from conducting longitudinal studies in younger populations with additional characterization of the underlying genetic abnormalities, along with other potential biomarkers, driving the overgrowth and onset of autistic symptoms at a critical time in the developmental process. Hence, it is of great significance to establish more effective biomarkers in order to facilitate diagnostics in patients 
before their conventional ASD symptoms become evident.

Future studies that include more than two time points of MRI data would be particularly helpful in the construction and comparison of ASD and normal brain development patterns. Yet, another promising method for understanding the relationship between brain development and ASD is to statistically analyze local 3D shapes and surfaces of the primary components of the brain and correlate these results to the common behavioral scores, such as the social responsiveness scale (SBS). However, correlating the results will be challenging, since such scales were developed as screening tools to qualitatively describe ASD severity, rather than to quantify the health deficits.

Diagnosing ASD with DTI The VBA, applied mostly to small and predominantly adolescent groups, has shown decreased FA in the major WM tracts of patients with ASD. The usefulness of VBA of DTI remains in doubt because this method depends on the size of the smoothing kernel and hinders confident conclusions. The TBSS, optimizing the VBA of DTI and thus outperforming the traditional VBA, have been used in the majority of cases to examine the whole brain in older children and adolescents. Most of the larger studies have reported reduced FA and increased diffusivity in the ASD groups. Also, the ROIbased DTI studies of the older population (older children, adolescents, and adults) versus their controls confirm the finding of decreased FA in various brain areas including the anterior cingulate, the superior temporal gyrus, and the temporal stem WM. However, since the ROIs target only specific components of particular WM tracts, conclusions cannot be drawn on the entire WM tract or other infrequently examined tracts. Nonetheless, studying the DTI of older children, adolescents, and adults using the VBA, TBSS, and ROI methods suggest that the ASD-related microstructural WM alterations differ depending on the age of the individuals studied.

Mostly, the deterministic, rather than the advanced probabilistic tractography is employed to study the DTI, and the bulk of these studies have focused on the WM ab- 
normalities in the uncinate fasciculus, the arcuate fasciculus, the cingulum bundle, the inferior longitudinal fasciculus, and the inferior fronto-occipital fasciculus. The abnormalities include lower FA and higher diffusivity (primarily, in children and adolescents) and altered macrostructure (mainly, in adults). The only ASD-related longitudinal DTI study to date [153] confirmed that at least during the first two years of life, a number of WM tracts seem to be affected by a dynamic process occurring in the brains of children who develop the ASD.

To conclude, although a variety of MRI-based studies consisting of small groups of individuals, ranging widely in age, present diverse clinical ASD symptoms compared to controls, these studies have yielded valuable, yet varying results. Multi-site studies with large patient groups that comprehensively characterize the clinical, behavioral, and cognitive symptoms may be one of the most important strategies for increasing the probability for the discovery of new, effective biomarkers. Future studies of particular importance will track brain maturation from infancy to adulthood in individuals at high risk of developing ASD by using multi-modality macro-/microstructural and functional brain images. By studying a larger, non-gender biased population of patients, it is anticipated that the "false positive" rate of ASD diagnosis will decrease.

\section{G. Motivation behind this work}

From the survey conducted above and the limitations discussed, it can be inferred that combining different imaging modalities would allow for better autism diagnosis and more certainty.So far, no work in the literature has proposed a diagnostic system for ASD that combined different modalities in addition to conducting analysis on local areas of the brain.

To overcome these limitations, and in order to obtain a robust classifier between autistic and control brains, this thesis proposes a novel CAD system that fuses the shape features extracted from both the cerebral cortex $(\mathrm{Cx})$ and cerebral white matter (CWM) 
of structural MR brain images along with features extracted using fMRI modality. Fusion between $\mathrm{Cx}$ and CWM in sMRI is conducted based on the recent findings suggesting that Cx changes in autism are related to CWM abnormalities [274]. Adding the fMRI modality is for combining both anatomical and functional information to better classify autistic individuals from controls. The CAD system starts with segmenting $\mathrm{Cx}$ and CWM using a 3D joint model that combines intensity, shape, and spatial information. Then, to accurately extract the 3D shape features of Cx, Spherical Harmonic (SPHARM) is applied to its re-constructed meshes, from which four metrics are derived for each mesh point; normal curvature, mean curvature, gaussian curvature, and $\mathrm{Cx}$ surface reconstruction error. To analyze the CWM shape, its gyri are extracted by propagating an orthogonal wave to their surfaces using 3D fast marching method and then their distance maps are computed as a shape feature. In addition, curvature analysis is estimated on CWM gyri surfaces using three shape features (curvedness, sharpness, and mean curvature). Moreover, a monetary reward fMRI experiment is conducted, and voxels that exhibited the most significant signal activation change due to the feedback event are used as features for classification between the two groups. Finally, all the extracted shape features are fed to a multi-level deep network for feature fusion and diagnosis.

This thesis integrated features from two imaging modalities sMRI and fMRI, while the long-term plan of the BioImaging laboratory is to integrate features from DTI with these two modalities as well.

\section{H. Dissertation Layout}

This thesis is organized as follows:

2- Chapter 2 introduces a novel framework for 3D brain segmentation from MR images. The framework is based on stripping the skull, followed by applying a 3D joint model that combines shape, intensity, and spatial information for brain segmentation. The framework has been tested on several databases that span different life stages in order to 
show its robustness.

3- Chapter 3 introduces a novel CAD system for autism diagnosis, which is the ultimate goal of this thesis. It is based on shape feature extraction from cerebral cortex $(\mathrm{Cx})$ and cerebral white matter (CWM) of structural MR images along with using fMRI modality for diagnosis. Finally, features from both modalities are fused for classification. Shape features from both structures were fused based on studies suggesting that neuropathological findings in regards to a putative minicolumnopathy and dysplastic $\mathrm{Cx}$ changes tend to magnify themselves by abnormal connections of CWM.

4- Chapter 4 concludes the dissertation and outlines possible points for future work. 


\section{CHAPTER II}

\section{SEGMENTATION OF MR BRAIN IMAGES USING ADAPTIVE SHAPE PRIOR AND HIGHER-ORDER MGRF}

This chapter introduces a new framework for the segmentation of different brain structures from 3D MR brain images at different life stages. The proposed segmentation framework is based on a shape prior built using a subset of co-aligned training images that is adapted during the segmentation process based on first- and second-order visual appearance characteristics of infant MRIs. These characteristics are described using voxel-wise image intensities and their spatial interaction features. To more accurately model the empirical grey level distribution of brain signals, especially for infant brains, a Linear Combination of Discrete Gaussians (LCDG) model having positive and negative components is used. To accurately account for the scan inhomogeneities, especially with infant brain MRIs, a higher-order Markov-Gibbs Random Field (MGRF) spatial interaction model that integrates third- and fourth- order families with a traditional second-order model is proposed. The proposed approach was tested and evaluated on 102 3D MR brain scans using three metrics: the Dice coefficient, the 95- percentile modified Hausdorff distance, and the absolute brain volume difference. Experimental results promise more accurate segmentation of MR brain images compared to current open source segmentation tools.

\section{A. Introduction}

Accurate delineation of brain tissues from Magnetic Resonance (MR) images is an essential step in clinical diagnostics, therapy evaluation, human brain mapping, and neuroscience [275]. In particular, segmenting a brain MR image contributes much to the analysis and treatment of brain injuries and disorders [276]. However, the brain MRI segmentation 
meets with challenges stemming from image noise, inhomogeneities, artifacts, such as partial volume effect and the discontinuities of boundaries due to similar visual appearance of adjacent brain structures. This work addresses brain MRI segmentation at different life stages, having the infancy stage the most complicated one due to many challenges such as reduced contrast, higher noise [277], and inverse contrast between the White Matter (WM) and Gray Matter (GM) in the infant brain MRIs [278], Figure 11. Segmentation of the brain at later stages may be based on only image intensity, because the contrast between different types of tissues is much better and the signal-to-noise ratios are improved, Figure 11.

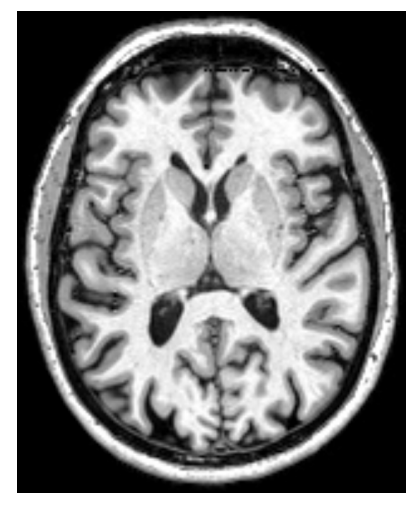

(a)

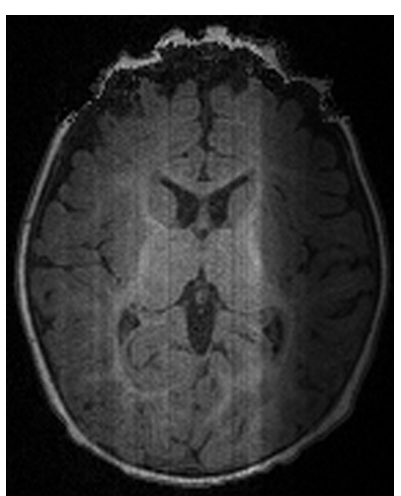

(b)

FIGURE 11: Example for a T1-weighted MRI for an adult (a) and an infant (b) brain.

Lower contrast between infant brain tissue classes stems from the fact that most of the WM is unmyelinated yet and its water content is close to the GM one. Moreover, both the WM and GM have the same intensity at about nine months of age [279]; hence, it is difficult to classify the infant brain tissues using only the intensity. Additionally, major partial volume effects occur due to the contrast inversion between the WM and GM compared to the adult brain MRI. The unmyelinated WM intensity is just between intensities for the GM and Cerebrospinal Fluid (CSF) tissues, so that partial volume averaging often misclassifies the average between the latter two tissues as the unmyelinated WM [278]. Furthermore, the MRI factors, such as long scan duration, small voxel size, and low signal-to-noise ratio (SNR) also hinder the infant brain imaging. High-resolution images are essential to 
show the infant brain structure because of its much smaller size compared to the adult brains [276]. However small-size voxels lead to noisy infant MRI with reduced contrast, i.e. with low contrast-to-noise ratio (CNR). To improve the CNR, the scan duration has to be increased; however, it is too risky for an infant to be anesthetized for a long period. As it is important to minimize scan times, usually infants are being fed and then wrapped to prevent movement [280]. One may expect that new imaging technologies such as parallel imaging or next-generation multi-channel imaging coils will decrease the scan time and increase the CNR and SNR. A large variety of segmentation techniques has been developed over the last two decades in order to address the brain MRI segmentation challenges. These techniques can be roughly classified into three main categories: (i) probabilistic, or statistical methods, (ii) atlas-based methods, and (iii) techniques based on deformable models.

\section{Probabilistic segmentation}

These algorithms involve prior models that describe the signal distributions of each brain structure. $\mathrm{Ng}$ et al. [281] segmented MR brain images using the unsupervised $\mathrm{K}$ means clustering of signals and an improved watershed algorithm. Signal clustering to produce an initial image segmentation before applying the improved watershed transform helped overcome the basic weaknesses of the latter, such as over-segmentation and sensitivity to false edges. A similar approach by Xue et al. [278] employed a parametric Gaussian density estimation with an Expectation-Maximization (EM) algorithm and constrained spatial homogeneity of the MR images with a Markov random field (MRF) prior. Partial volume averaging effects have been eliminated by predicting the misclassification (e.g., of an 'averaged' CSF and GM into an intensity similar to WM). Song et al. [282] proposed a probabilistic neural network (PNN) for segmenting the brain MRI. Probability density functions of the brain tissues were estimated from reference vectors generated by a self-organizing map (SOM). To reduce the partial volume averaging effects, weighting 
factors were added to the summation layer's patterns in a weighted probabilistic neural network (WPNN) and soft labeling was performed by a supervised Bayesian classifier. An automated MRI brain segmentation method by Mayer et al. [283] combined spatial and intensity features into a high-dimensional feature space. An adaptive mean-shift classifier extracted a set of convergence modes, i.e. high-density points of a feature space, being good candidates for intensity-based classification. Brain tissues were classified by an intensity-based mode clustering. This approach was very effective with non-convex clustering. Fang et al. [284] developed a tree metrics (TM)-based graph cut algorithm to segment the MRI brain tissues. After a brain MR image is classified using the TM, the goal labeling is inferred by "tree-cutting". In contrast to most of conventional iterative methods like the EM-based ones, which produce only locally optimal labeling, this algorithm needs no more than one sweep to generate the globally optimal labeling with respect to the TM. An automated segmentation approach by Ortiz et al. [285] classified the brain tissue with no prior information. The segmentation consisted of feature extraction and classification. Extracted first order (pixel/voxel-wise), second order (pair-wise), moment, and scale-invariant features were classified by growing hierarchical SOMs (GHSOM). A method was proposed by Patenaude at al. [286] that used manually labeled image training data. It utilized the principles of both the active Shape and appearance models within a Bayesian framework, allowing probabilistic relationships between shape and intensity to be fully used. Li et al. [287] proposed a 3D MGRF model for the segmentation of brain MR images to avoid the shortcomings of the 2D model which is not able to fully capture the spatial information, especially among the slices. An initial segmentation was first obtained by k-means clustering in order to reduce the extensive computations required by the MGRF model. The Iterated Conditional Modes (ICM) algorithm was finally applied to obtain the optimal solution under maximum a posteriori (MAP) criterion. A non-parametric adaptive mean-shift algorithm was proposed by Janney et al. [288] for brain tissue segmentation. It clustered the joint spatial-intensity feature space, followed by a phase of intensity-based 
mode clustering into the brain tissue types. The algorithm could deal with non-convex clusters, and also produced convergence modes that were better applicants for intensitybased segmentation than the initial voxels. Weber et al. [289] segmented the brain tissues using FSL-FAST free software that is based on K-means clustering which provided initial segmentation, followed by the EM algorithm for bias field correction. In order to speed up the process, parallelization to any eligible parts of the software was applied, which needed some adaptation to the algorithms in order to maintain the accuracy obtained by the software package. Mahmood et al. [290] proposed an unsupervised framework for brain tissue segmentation using a combination of Bayesian-based adaptive mean shift that clustered the tissues in the joint spatial-intensity feature space, and fuzzy c-means that is initialized with a priori spatial tissue probability maps to assign the clusters into three tissue types; WM, GM, and CSF.

Infant brain segmentation using statistical-based methods was also addressed in literature. Automated segmentation of brain structures, such as WM, CSF, Central GM (CEGM), and Cortical GM (COGM) was conducted by Anbeek et al. [291] using T2weighted and inversion recovery (IR) MRI of the neonatal brains. Probability maps to segment each brain tissue class with a $K$-nearest neighbor (KNN) classifier using voxel intensities and coordinates as features were constructed manually. A multi-label segmentation process combined the obtained classes. Wang et al. [292] segmented T1, T2 and diffusion-weighted brain images using a sparse representation of the complementary tissue distribution. Initially, the brain tissue was segmented into different structures using a patch-based technique with a library of multi-modality images, having been aligned with their ground-truth segmentation maps. Then the segmentation was refined by integrating geometric constraints. Wang et al. [293] employed a random forest technique to integrate features from different modalities for brain tissue segmentation in infants along with probability maps of GM and CWM. Infant brain segmentation using shape priors was also addressed in the literature [294-296]. Zhang et al. [297] proposed a deep convolutional 
neural networks (CNNs) approach for segmenting the neonatal brains from multi-modal MR images, generating the segmentation maps as outputs. The multiple intermediate layers included many operations such as convolution, pooling, and normalization in order to capture the highly nonlinear mappings between inputs and outputs. Moeskops et al. [298] segmented neonatal brains int WM, GM, and CSF using supervised voxel classification. First, voxels that could easily be assigned to one of the three types are labeled using twoclass classification for each tissue type separately. A multi-class classification was then performed.

Statistical-based techniques are generally fast to implement compared to other segmentation methods. However, they depend only on predefined probability models that cannot fit all of the possible real data distributions. This is due to the fact that actual intensity distributions of brain structures are greatly affected by several factors, such as the unique patient and scanner along with scanning parameters. Also, due to the similar intensities (gray levels) for the different brain tissue structures of the infant MR brain images, segmentation techniques only based on the intensity remain inaccurate.

\section{Atlas-based segmentation}

Atlas-based approaches have emerged as powerful segmentation tools. These approaches are based on a priori knowledge about brain structures, and treat the segmentation problem as a registration task. Ashburner et al. [299] introduced a generative framework that combined image registration, tissue classification, and bias correction. Their framework incorporated a smooth intensity variation and nonlinear registration with tissue probability maps using mixture of Gaussians. Pohl et al. [300] introduced a Bayesian model for simultaneous segmentation and registration. Their framework tried to exploit complementary aspects of registration and segmentation problems. In order to account for different physiological (patient size and weight) and scanning (scanner type and data acquisition protocol) parameters, Han et al. [301] introduced an intensity re-normalization procedure 
to adjust the prior atlas intensity model to new input data to overcome the problems stemming from using training data acquired from a different scanner that was used for the test data. The re-normalization process updated the class-conditional densities for each atlas structure by applying a multi-linear atlas-image registration and histogram matching. Artaechevarria et al. [302] proposed a generalized local weighting voting scheme in which the fusion weights were adapted for each voxel based on local estimation of the segmentation performance. The local weighting voting outperformed traditional global strategies that estimate a single value for the segmentation accuracy for the whole image. Sabuncu et al. [303] proposed an automated label fusion segmentation technique. In order to capture greater inter-subject anatomical variability, each training data set was individually coregistered to the test data set. Then, a nonparametric probabilistic model was employed to fuse the training labels to compute the final segmentation. Morin et al. [304] presented an atlas-based segmentation framework using random walks that combined registration and labeling propagation steps. They used a generative model to provide pixel label probabilities to improve the segmentation for high-confidence labels. To match the target images with atlas images, they used the Affine-Scale Invariant Feature Transform (ASIFT) [305] and Speeded Up Robust Features (SURF) [306] registration techniques. In order to avoid segmentation errors produced by registration imperfection, Lötjönen et al. [307] introduced an optimized pipeline for multi-atlas brain MRI segmentation. They introduced two approaches that combine multi-atlas segmentation and intensity modeling based on using EM and graph cuts for optimization. First, they registered all atlases to the target data and a majority voting was applied to predict the segmentation of the target image. Then, the segmentation was improved using the intensity modeling as a post-processing step. Lijn et al. [308] introduced a segmentation method based on the combination of spatial features and appearance models. They generated a spatial probability map that was obtained from multiple atlas-target image registrations, to implement the spatial model. The tissue appearance was modeled by a KNN classifier using Gaussian scale-space features. Then, a 
Bayesian framework was used to combine both spatial and appearance models and a graphcut approach [309] was used for optimization. Ledig et al. [310] introduced a framework for labeling whole brain scans by incorporating a global and stationary MRF to ensure consistency of the neighborhood relations between structures with an a priori defined model.

Segmentation of neonatal brains was also conducted in the literature using atlasbased techniques. A method for the segmentation of axial neonatal brain MRI that combined multi-atlas-based segmentation and supervised voxel classification was proposed, [311], in order to segment eight different tissue classes, namely cortical grey matter (CoGM), unmyelinated white matter (UWM), brainstem, cerebellum, ventricles, cerebrospinal fluid in the extra-cerebral space (CSF), basal ganglia, and myelinated white matter (MWM). Some approaches use longitudinal scans at a late-time-point age, where the contrast is much better between different tissue types, from which probabilistic atlases are constructed to guide segmentation of neonatal images [312,313]. Cherel et al. [314] employed a subject-specific atlas that is based on manually segmented data for the segmentation of brain tissue classes. The atlases were incorporated with single-atlas expectation maximization (EM) method.

Atlas-based segmentation techniques show more accuracy with respect to statisticalbased techniques. Nevertheless, they are still challenged by atlas selection, combination, and the associated heavy computation time. Another major drawback of atlas-based segmentation algorithms is their dependency on the selected features that will be used to link between the test subject and the prior (training) data used in the construction of the atlas. For example most of the current techniques use signal intensity to find the correspondence between the data to be segmented and the prior atlas. This may lead to inaccurate segmentation results as signal intensities (gray levels) vary due to many factors such as the patient's age, and the scanning protocol. 


\section{Deformable models-based segmentation}

In order to obtain continuous segmentation of brain structures, deformable boundaries have also been recognized as more accurate segmentation techniques of MR brain tissues. Angelini et al. [315] introduced a multi-phase level set framework for automated segmentation of brain MRIs. The segmentation of the brain tissues (WM, GM and CSF) was solely based on homogeneity (average grey level) measures. To avoid the need for any prior information and to speed up numerical calculation, a random seed for initialization of the deformable boundaries was used. Colliot et al. [316] proposed a deformable model-based approach that used spatial constraints, represented as fuzzy subsets of the 3D image space, as an external force to control the boundary evolution. To avoid manual selection of the model parameters, a training step was required to estimate the spatial constraints parameters. Miri et al. [317] introduced a topology-preserving deformable model framework for the segmentation of brain MRI. They employed photometric constraints to guide the deformable model deformations to iteratively reclassify the points located at the evolving boundaries. A deformable model approach for the segmentation of brain regions from MR images was proposed by Liu et al. [318]. The deformable contour was implicitly represented by a set of Wendland's radial basis functions (RBFs) and was evolved by iterative updates of the locations of the RBFs. The updates of the RBFs locations were controlled by an external force that considered the intensity contrast across boundaries. Huang et al. [319] introduced an automated, hybrid deformable model framework that integrated both image edge geometry and voxel statistics features to regularize the convergence of the deformable contour. Del Fresno et al. [320] described a hybrid method that combined region growing and deformable models for segmentation of different structures in head MRI and Computed Tomography CT scans. Their approach used a Region-Growing (RG) algorithm to compute an approximation of the objects. This was followed by generating closed and oriented surface meshes to enclose the region of interest. To improve the segmentation of noisy images, local neighborhood features of each voxel of the region boundary 
were used. The deformable model method geometry was constructed using the RG-list of boundary voxels generating a hole-free surface mesh. To better detect the structures of interest, the user could select few seeds for RG initial segmentation. Wang et al. [321] proposed a multi-phase level set framework to segment brain MR images with intensity inhomogeneity. They modeled the local image intensities using Gaussian distributions with different means and variances. Then, a variational approach minimized an energy function to compute the means and variances that would guide the contour evolution towards the target boundaries. Bourouis et al. [322] developed a level set framework for segmenting brain tissues. Their framework employed an image registration step and a classification step for the initialization of the deformable boundary. The boundary evolution was controlled by a speed function that accounted for both boundary- and region-based properties. Ciofolo et al. [323] developed an automated framework based on level sets for simultaneous segmentation of multiple structures from brain MRIs. The evolution of each level set was driven by a fuzzy decision system that combined three factors: intensity distribution of the 3D MR volume, the relative position of the evolving contours, and a priori knowledge provided by an anatomical atlas. Wang et al. [324] proposed a multi-layer background subtraction technique with a seed region growing approach which used local texture features represented by local binary patterns (LBP) and photometric invariant color measurements in RGB color space for brain MRI segmentation. Zhao et al. [325] segmented brain tissues using a method that is adapted from Chan and Vese model, named automatic threshold level set without edges. Threshold were obtained by fuzzy c-mean algorithm.

Segmentation using deformable models was also exploited in infant brains, [292, 293, 312, 313].

The main advantage of deformable model-based segmentation techniques is the ability to segment connected (non-scattered) objects more accurately than the other segmentation methods. However, the accuracy of this method is based on the accurate design of the guiding forces (statistical, geometric, etc.) in addition to the initialization of the 
model.

In summary, brain segmentation work found in the literature suffers from many drawbacks as mentioned before for each type of segmentation. Moreover, infant brain segmentation techniques depend on either multiple modalities which lengthens the processing time, or on longitudinal studies which are not always available for research purposes. Also, the reduction in contrast between CWM and other structures hardens the issue of preserving its edges by most of the available techniques

To overcome the aforementioned limitations, this thesis proposes a novel technique for brain segmentation from MR images. Adaptive probabilistic shape models for the shape and first-order visual appearance of MRI data are employed to initialize the segmentation. This is then combined with a novel higher-order Markov Gibbs Random Field (MGRF) spatial interaction model (up to fourth order) with analytic estimation of potentials. This joint model guarantees increasing the segmentation accuracy by accounting for the large inhomogeneities and noise, especially in infant brain MRI data. Also, the analytic estimation of potentials generalizes the proposed model to different MRI subjects, unlike using empirical values in most of the work present in the literature which would require manual setting for each subject. The strength of the proposed algorithm lies in the fact that it neither depends on multiple modalities for acquiring images nor on longitudinal studies that are not always available for research purposes.

\section{B. Methods}

The proposed framework is based on two main components: (i) brain extraction (skull stripping); and (ii) brain segmentation. Figure 12 illustrates the proposed framework. Details of the framework are outlined below. 

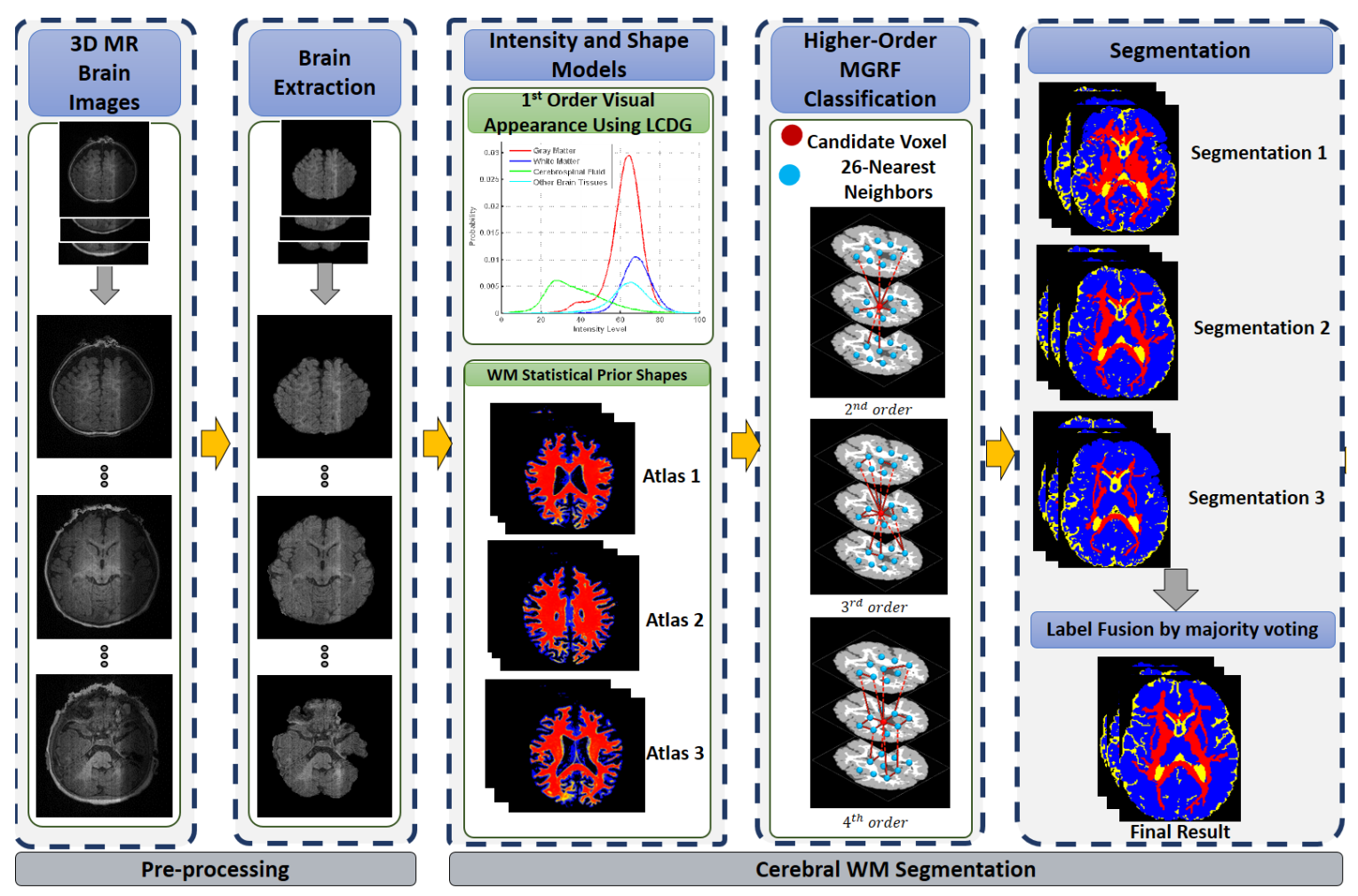

FIGURE 12: The proposed segmentation framework.

\section{Preprocessing}

Before segmentation of brain scans takes place, a novel framework is applied for the automated extraction of the brain $[326,327]$. The proposed approach is primarily based on the integration of a stochastic model (a two-level Markov-Gibbs random field (MGRF)) that serves to learn the visual appearance of the brain texture, and a geometric model (the brain iso-surfaces) that preserves the brain geometry during the extraction process. The proposed framework consists of three main steps: (i) Following bias correction of the brain, a new 3D MGRF having a 26-pairwise interaction model is applied to enhance the homogeneity of MR images and preserve the 3D edges between different brain tissues; (ii) The nonbrain tissue found in the MR images is initially removed using the brain extraction tool (BET), and then the brain is parceled to nested iso-surfaces using a fast marching level set method; (iii) Finally, a classification step is applied in order to accurately remove the remaining parts of the skull without distorting the brain geometry. The classification of each 
voxel found on the iso-surfaces is made based on the first- and second-order visual appearance features. The first-order visual appearance is estimated using a linear combination of discrete Gaussians (LCDG) to model the intensity distribution of the brain signals. The second-order visual appearance is constructed by using an MGRF model with analytically estimated parameters.

\section{Joint MGRF Model of MR Brain Images}

Let $\mathbf{R}=\{(x, y, z): 0 \leq x \leq X-1,0 \leq y \leq Y-1,0 \leq z \leq Z-1\}$; $\mathbf{Q}=\{0,1, \ldots, Q-1\}$; and $\mathbf{L}=\{0, \ldots, L\}$ denote a finite 3D arithmetic lattice of the size of $X Y Z$ supporting grayscale images and their region (segmentation) maps, a finite set of $Q$ integer gray values, and a set of region labels $L$, respectively. Let $\mathbf{g}=\left\{g_{x, y, z}\right.$ : $\left.(x, y, z) \in \mathbf{R} ; g_{x, y, z} \in \mathbf{Q}\right\}$ and $\mathbf{m}=\left\{m_{x, y, z}:(x, y, z) \in \mathbf{R} ; m_{x, y, z} \in \mathbf{L}\right\}$ be a grayscale image taking values from $\mathbf{Q}$, i.e., $\mathbf{g}: \mathbf{R} \rightarrow \mathbf{Q}$, and a region map taking values from $\mathbf{L}$, i.e., $\mathbf{m}: \mathbf{R} \rightarrow \mathbf{L}$, respectively. An input brain image, $\mathbf{g}$, co-aligned to the training data base, and its map, $\mathbf{m}$, are described with a joint probability model: $P(\mathbf{g}, \mathbf{m})=P(\mathbf{g} \mid \mathbf{m}) P(\mathbf{m})$, which combines a conditional distribution of the images given the map $P(\mathbf{g} \mid \mathbf{m})$, and an unconditional probability distribution of maps $P(\mathbf{m})=P_{\mathrm{sp}}(\mathbf{m}) P_{\mathbf{V}}(\mathbf{m})$. Here, $P_{\mathrm{sp}}(\mathbf{m})$ denotes a weighted shape prior, and $P_{\mathbf{V}}(\mathbf{m})$ is a Gibbs probability distribution with potentials V, which specifies a MGRF model of spatially homogeneous maps $\mathbf{m}$. Details of the model's components are outlined below.

a. Adaptive Shape Model $P_{\mathrm{sp}}(\mathbf{m})$ To start the segmentation process, a database is created, where expected shapes of each brain label are constrained with an adaptive probabilistic shape prior. To create the shape database, a training set of images, collected for different subjects (15 data sets, not included as test subjects), are co-aligned by 3D affine transformations with 12 degrees of freedom (three for the 3D translation, three for the 3D rotation, three for the 3D scaling, and three for the 3D shearing) in a way that maximizes their Mutual Information (MI) [328]. The 15 subjects were spanning different ages and 
included control and autistic subjects (to avoid being biased to a certain group). This set is then subdivided into three atlases based on the normalized cross correlation measure. This is done in order to improve the segmentation accuracy by integrating each atlas with both the intensity and spatial models (to be discussed in the following subsections), and finally applying the majority voting criterion to determine the class of each voxel. For each input MR data to be segmented, the shape prior is constructed by an adaptive process guided by the visual appearance features of the input MRI data [329-331]. The shape prior is a spatially variant independent random field of region labels for the co-aligned data:

$$
P_{\mathrm{sp}}(\mathbf{m})=\prod_{(x, y, z) \in \mathrm{R}} p_{\mathrm{sp}: x, y, z}\left(m_{x, y, z}\right)
$$

where $p_{\mathrm{sp}: x, y, z}(l)$ is the voxel-wise empirical probabilities for each brain label $l \in \mathbf{L}$. First, the normalized cross correlation similarity coefficient is used to select the subject from the shape database that has the best match with the input subject (i.e., highest similarity). The selected subject is then used as a reference prototype to co-align the input subject using the 3D affine transformation described above. In order to estimate the shape prior probabilities for each voxel in the test subject, the steps summarized in Algorithm 1 are followed.

b. First-Order Intensity Model $P(\mathbf{g} \mid \mathbf{m})$ The first-order visual appearance of each brain label is modeled by separating a mixed distribution of voxel intensities of the infant brain MRIs into individual components associated with the dominant modes of the mixture. The latter is precisely approximated with a Linear Combinations of Discrete Gaussians (LCDG) [332] with positive and negative components, which is based on a modified version of the classical Expectation-Maximization (EM) algorithm.

Let $\boldsymbol{\Psi}_{\theta}=(\psi(q \mid \theta): q \in \mathbf{Q})$ denote a discrete Gaussian (DG) with parameters $\theta=(\mu, \sigma)$, integrating a continuous 1D Gaussian density with mean $\mu$ and variance $\sigma^{2}$ over successive gray level intervals. The LCDG with four dominant positive DGs and $C_{\mathrm{p}} \geq 4$ positive and $C_{\mathrm{n}} \geq 0$ negative subordinate DGs is [332]: 


$$
P_{\mathbf{w}, \boldsymbol{\Theta}}(q)=\sum_{k=1}^{C_{\mathrm{p}}} w_{\mathrm{p}: k} \psi\left(q \mid \theta_{\mathrm{p}: k}\right)-\sum_{\kappa=1}^{C_{\mathrm{n}}} w_{\mathrm{n}: \kappa} \psi\left(q \mid \theta_{\mathrm{n}: \kappa}\right)
$$

where all the weights $\mathbf{w}=\left[w_{\mathrm{p}: k}, w_{\mathrm{n}: \kappa}\right]$ are non-negative and meet an obvious constraint $\sum_{k=1}^{C_{\mathrm{p}}} w_{\mathrm{p}: k}-\sum_{\kappa=1}^{C_{\mathrm{n}}} w_{\mathrm{n}: \kappa}=1$. All LCDG parameters, including the DGs numbers, are estimated from the mixed empirical distribution to be modeled using the modified EM algorithm [332].

c. MGRF Model With Second- and Higher-order Cliques $P_{\mathbf{V}}(\mathbf{m})$ In addition to the first-order visual appearance model, the spatial interactions between the brain voxels are also taken into account. Using spatial models that are second-order-clique based, (e.g., [333]), will not enable accounting for the large inhomogeneity of brain MR images, especially for infants. Therefore, in this work we propose a higher-order Markov-Gibbs Random Field (MGRF) spatial interaction model that adds the families of the triple and quad cliques to the pairwise cliques (Figure 13(b,c)) with analytical estimation of the potentials. The proposed approach has the ability to account for the large inhomogeneity of infant MRIs, thus, reducing the noise effects and increasing the segmentation accuracy $[329,330]$. Details of the proposed higher-order MGRF model are described below.

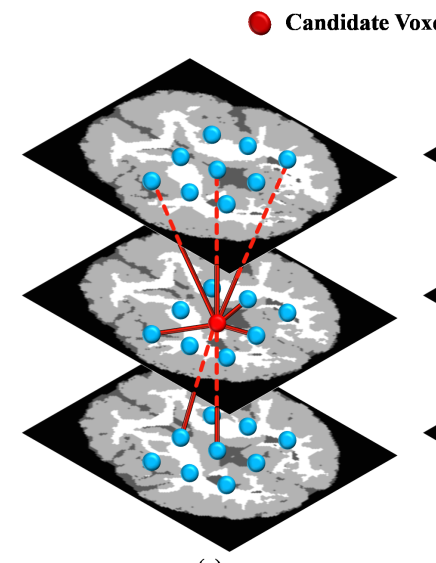

(a)

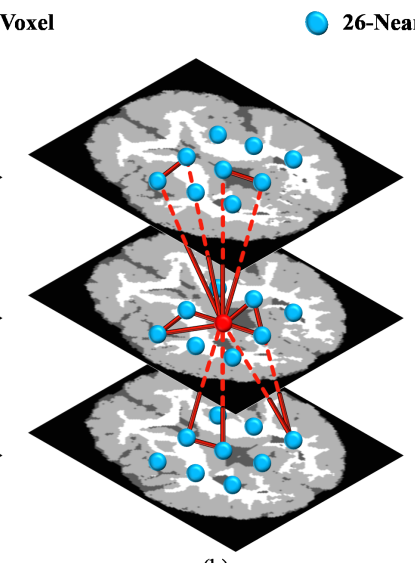

(b)

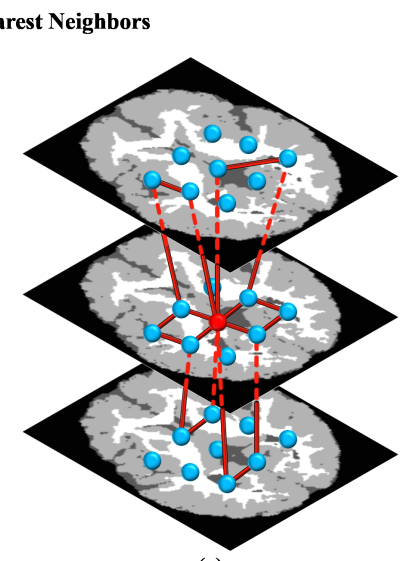

(c)

FIGURE 13: Samples of the second- $(a)$, third- $(b)$, and fourth-order $(c)$ cliques for the 26-neighborhood (graph cliques are shown in different colors for visualization purpose). 
Let $\mathbf{C}_{a}$ denote a family of $s$-order cliques of an interaction graph with nodes in the 3D lattice sites $(x, y, z)$ and edges connecting the interacting, or interdependent, sites (see Figure 13). To account for large variations of the infant MRIs, the label interactions are modeled by a spatially homogeneous MGRF with up to fourth-order interactions over the nearest 26-neighborhoods of voxels:

$$
P_{\mathbf{V}}(\mathbf{m})=\frac{1}{Z_{\mathbf{V}}} \exp \left(\sum_{a=1}^{A} \sum_{\mathbf{c} \in \mathbf{C}_{a}} V_{a}(\mathbf{m}(x, y, z):(x, y, z) \in \mathbf{c})\right)
$$

where $A$ clique families describe the geometry of the graph interactions,

$$
\mathbf{V}=\left[V_{a}:\{0, \ldots, L\} \rightarrow(-\infty, \infty): a=1, \ldots, A\right] \text { is a collection of Gibbs poten- }
$$

tial functions $V_{a}$ for the families $\mathbf{C}_{a}$, and the partition function $Z_{\mathbf{V}}$ normalizes the probabilities over the parent population $\mathbb{M}=\{0, \ldots, L\}^{X Y Z}$ of all maps. An initial region map $\mathbf{m}$, obtained by the voxel-wise classification, allows for analytically approximating the maximum likelihood estimates of the potentials and computing the voxel-wise probabilities of the region labels. For symmetry sake, only equality or inequality of the labels in clique c is taken into account. The second- third- and forth-order potentials are given by Equations (9), (10), and (11), respectively:

$$
V_{a}\left(m_{\mathbf{p}_{1}}, m_{\mathbf{p}_{2}}\right)=\left\{\begin{array}{cl}
V_{2: a: \text { eq }} & \text { if } m_{\mathbf{p}_{1}}=m_{\mathbf{p}_{2}} \\
-V_{2: a: e q} & \text { otherwise }
\end{array}\right.
$$

where $V_{2: a: \text { eq }}=-V_{2: a: \text { ne }}=4\left(F_{a: \text { eq }}\left(\mathbf{m}^{\circ}\right)-\frac{1}{2}\right)$, and $\mathbf{F}\left(\mathbf{m}^{\circ}\right)=\left[\rho_{a} F_{a}\left(\mu_{1}, \ldots, \mu_{s} \mid \mathbf{m}^{\circ}\right)\right.$ : $\left.\left(\mu_{1}, \ldots, \mu_{s}\right) \in\{0, \ldots, L\}^{s} ; a=1, \ldots, A\right]$ is the collection of scaled relative frequencies of co-occurrences of configurations $\left(\mu_{1}, \ldots, \mu_{s}\right)$ of the labels in the cliques of each family $\mathrm{C}_{a}$ over a given training map $\mathbf{m}^{\circ}$.

$$
V_{a}\left(m_{\mathbf{p}_{1}}, m_{\mathbf{p}_{2}}, m_{\mathbf{p}_{3}}\right)=\left\{\begin{array}{cl}
V_{3: a: \mathrm{eq}_{3}} & \text { if } m_{\mathbf{p}_{1}}=m_{\mathbf{p}_{2}}=m_{\mathbf{p}_{3}} \\
-V_{3: a: \mathrm{eq}_{3}} & \text { otherwise }
\end{array}\right.
$$


where $V_{3: a: \mathrm{eq}_{3}}=-V_{3: a: \mathrm{eq}_{2}}=\frac{16}{3}\left(F_{a: \mathrm{eq}_{3}}\left(\mathbf{m}^{\circ}\right)-\frac{1}{4}\right)$

$$
V_{a}\left(m_{\mathbf{p}_{1}}, m_{\mathbf{p}_{2}}, m_{\mathbf{p}_{3}}, m_{\mathbf{p}_{4}}\right)= \begin{cases}V_{4: a: \mathrm{eq}_{4}} & \text { if } 4 \text { equal labels } \\ V_{4: a: \mathrm{eq}_{3}} & \text { if } 3 \text { equal labels } \\ -\left(V_{4: a: \mathrm{eq}_{3}}+V_{4: a: \mathrm{eq}_{4}}\right) & \text { otherwise }\end{cases}
$$

where

$$
\begin{aligned}
& V_{4: a: \mathrm{eq}_{4}}=\lambda^{*}\left(F_{a: \mathrm{eq}_{4}}\left(\mathbf{m}^{\circ}\right)-\frac{1}{8}\right) \\
& V_{4: a: \mathrm{eq}_{3}}=\lambda^{*}\left(F_{a: \mathrm{eq}_{3}}\left(\mathbf{m}^{\circ}\right)-\frac{1}{2}\right) \\
& V_{4: a: \mathrm{eq}_{2}}=\lambda^{*}\left(F_{a: \mathrm{eq}_{2}}\left(\mathbf{m}^{\circ}\right)-\frac{3}{8}\right)=-\left(V_{4: a: \mathrm{eq}_{4}}+V_{4: a: \mathrm{eq}_{3}}\right)
\end{aligned}
$$

and

$$
\lambda^{*}=\frac{\sum_{a=1}^{A}\left(\left(F_{a: \mathrm{eq}_{4}}\left(\mathbf{m}^{\circ}\right)-\frac{1}{8}\right)^{2}+\left(F_{a: \mathrm{eq}_{3}}\left(\mathbf{m}^{\circ}\right)-\frac{1}{2}\right)^{2}+\left(F_{a: \mathrm{eq}_{2}}\left(\mathbf{m}^{\circ}\right)-\frac{3}{8}\right)^{2}\right)}{\sum_{a=1}^{A}\left(\frac{7}{64}\left(F_{a: \mathrm{eq}_{4}}\left(\mathbf{m}^{\circ}\right)-\frac{1}{8}\right)^{2}+\frac{1}{4}\left(F_{a: \mathrm{eq}_{3}}\left(\mathbf{m}^{\circ}\right)-\frac{1}{2}\right)^{2}+\frac{15}{64}\left(F_{a: \mathrm{eq}_{2}}\left(\mathbf{m}^{\circ}\right)-\frac{3}{8}\right)^{2}\right)}
$$

where $m_{\mathbf{p}_{i}}$ is the region map label at the voxel $\mathbf{p}_{i}=\left(x_{i}, y_{i}, z_{i}\right)$. The proposed analytical approximation of the Gibbs potentials from a given map $\mathbf{m}$ extends earlier second-order MGRFs (e.g., [333]) to the higher-order models. Finally, the region map $m$ is improved using Iterative Conditional Mode (ICM) algorithm [334] that maximizes the probabilities of the $3 \mathrm{D}$ joint model. The complete steps of our segmentation framework are summarized in Algorithm 1.

\section{Experimental Results}

The proposed segmentation framework was tested on different databases at different ages to show its generality and robustness. Since the ultimate goal of this thesis is to develop a novel CAD system for autism diagnosis, segmentation was validated on control as well as autistic subjects. Twenty one autistic subjects and their matched controls were analyzed from the Kennedy Krieger Institute (KKI)(8-12.8 years). Moreover, 10 autistic subjects and their matched controls from the university of California (UCLA) (8.4-17.9 years) and 10 autistic subjects and their matched controls from the NYU Langone Medical 
Center (6.5-39.1 years) [335] were analyzed. Also, infants from the NDAR/IBIS database (10 autism, 10 controls, aged 6 months) [336] were segmented using the proposed framework.

All the scans are T1-weighted, and obtained from the following sources: (1) The Infant Brain Imaging Study (hereafter, IBIS), a multisite, longitudinal study of infants at high risk of developing autism. These data are available from the National Database for Autism Research (NDAR). (2) Data from the Kennedy Krieger Institute, University of California, and NYU Langone Medical Center (hereafter, KKI, UCLA, and NYU respectively), made available through the Autism Brain Imaging Data Exchange [335].

IBIS data comprised T1-weighted images, and were acquired on a 3 tesla scanner with TR $=2400$ millisecond, $\mathrm{TE}=3.16$ millisecond TI = 1200 millisecond , and flip angle $=8.160$ sagittal slices were acquired at 1 millimeter thickness, with each slice being $224 \times$ 256 pixels with 1 millimeter resolution.

KKI data includes children with ASD as well as controls. Participants were between 8 and 13 years of age when scanned. T1-weighted images were acquired on a 3 tesla Philips Achieva with $\mathrm{TR}=8$ millisecond, $\mathrm{TE}=3.7$ millisecond, and flip angle $=8.128$ sagittal slices were acquired at 1.33 millimeter thickness. The pixels of each $256 \times 256$ slice were 1 millimeter each side.

UCLA data includes participants between 8.4 and 17.9 years of age when scanned. T1-weighted images were acquired using MPRAGE with TR $=2300$ millisecond, $\mathrm{TE}=$ 2.84 millisecond, and flip angle $=9$. Sagittal slices were acquired at 1.2 millimeter thickness. The pixels of each $256 \times 256$ slice were 1 millimeter each side.

NYU data includes participants between 6.5 and 39.1 years of age when scanned. T1-weighted images were acquired on a 3 tesla Allegra with $\mathrm{TR}=2530$ millisecond, $\mathrm{TE}=$ 3.25 millisecond, and flip angle $=7$. Sagittal slices were acquired at 1.33 millimeter thickness. The pixels of each $256 \times 256$ slice were 1.3 millimeter, and 1 millimeter respectively.

The proposed segmentation approach was evaluated on all subjects above using 
their manually segmented ground truth created by an MR expert. Results in the upcoming pages show that the joint model combining the intensity, spatial, and shape information is in general a better performer than having only one or two of the three models. The intensity information alone, or having it combined with the shape information, often fail to capture fine details, especially with infant MRI scans, where contrast is extremely poor between different tissue types, Figure 14 . This is enhanced after using higher-order MGRF, where edges of different tissue types are better retained. Figures 15, 16, and 17 show more segmentation results using the proposed framework from the different databases used in this study.

The segmentation performance was evaluated using 3 metrics: $(i)$ the Dice similarity coefficient (DSC) [337], (ii) the 95-percentile modified Hausdorff distance (MHD) [338], and (iii) the absolute brain volume difference (ABVD). Metrics were computed by comparing the ground truth segmentation to results from the proposed approach. Tables 1, 2, and 3 summarize the accuracy results obtained using the three metrics on the different databases.

The performance of the proposed segmentation approach is highlighted by comparing it against the software package (iBEAT) [339], that performs bias correction followed by brain segmentation. Segmentation accuracies for the iBEAT results are also summarized in Tables 1, 2, and 3. These results emphasize the efficiency of the proposed approach that is required for the next phase of the proposed CAD system. In addition, the average time to segment a brain volume using the proposed approach is less than a minute, whereas it takes around 40 minutes using iBEAT. Accuracies are reported for CWM and cerebral gray matter (CGM).

\section{Summary and Conclusion}

This chapter introduced a novel framework for 3D brain segmentation from MR images. The framework is based on stripping the skull, followed by applying a 3D joint model that combines shape, intensity, and spatial information for brain segmentation. Adaptive 
A
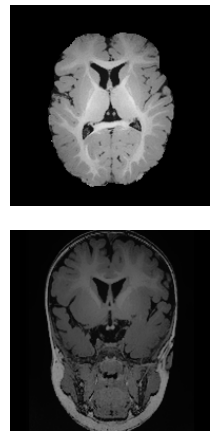

$\mathrm{S}$

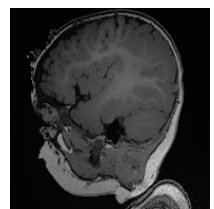

(a)
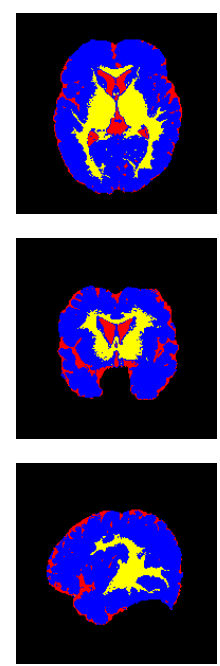

(b)
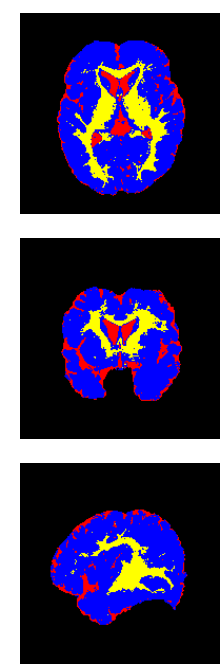

(c)
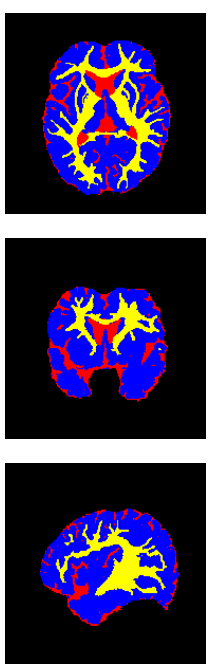

(d)
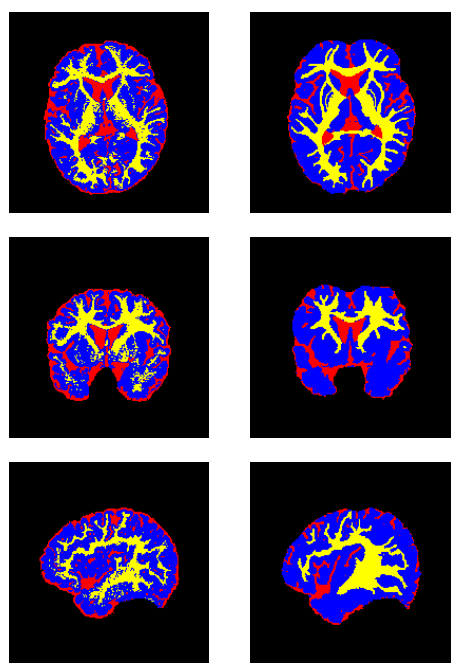

(e)
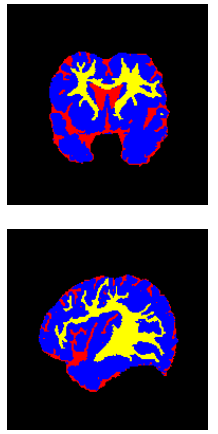

(f)

FIGURE 14: Segmentation results projected onto axial, coronal, and sagittal planes for a sample from the IBIS database for infants: (a) original MRIs after skull stripping, and the segmentation using (b) intensity model alone; (c) intensity and shape models (d) intensity, shape, and higher-order MGRF models; and (e) the iBEAT method. Ground truth is shown in (f). CWM is in yellow, GM in blue, and cerebrospinal fluid (CSF) is in red for visualization purposes. All subjects are bias-corrected prior to segmentation using iBEAT or the proposed method. It is clear that the intensity model alone (b) is not sufficient for segmentation, since the contrast is extremely low between CWM and GM. Results were further enhanced using the joint model (d).

probabilistic shape models for the shape and first-order visual appearance of MRI data are employed to initialize the segmentation. This is then combined with a novel higher-order Markov Gibbs Random Field (MGRF) spatial interaction model (up to fourth order) with analytic estimation of potentials. This joint model guarantees increasing the segmentation accuracy by accounting for the large inhomogeneities and noise, especially in infant brain MRI data. The strength of the proposed algorithm lies in the fact that it neither depends on multiple modalities for acquiring images nor on longitudinal studies that are not always available for research purposes. It was tested on several databases at different life stages, 

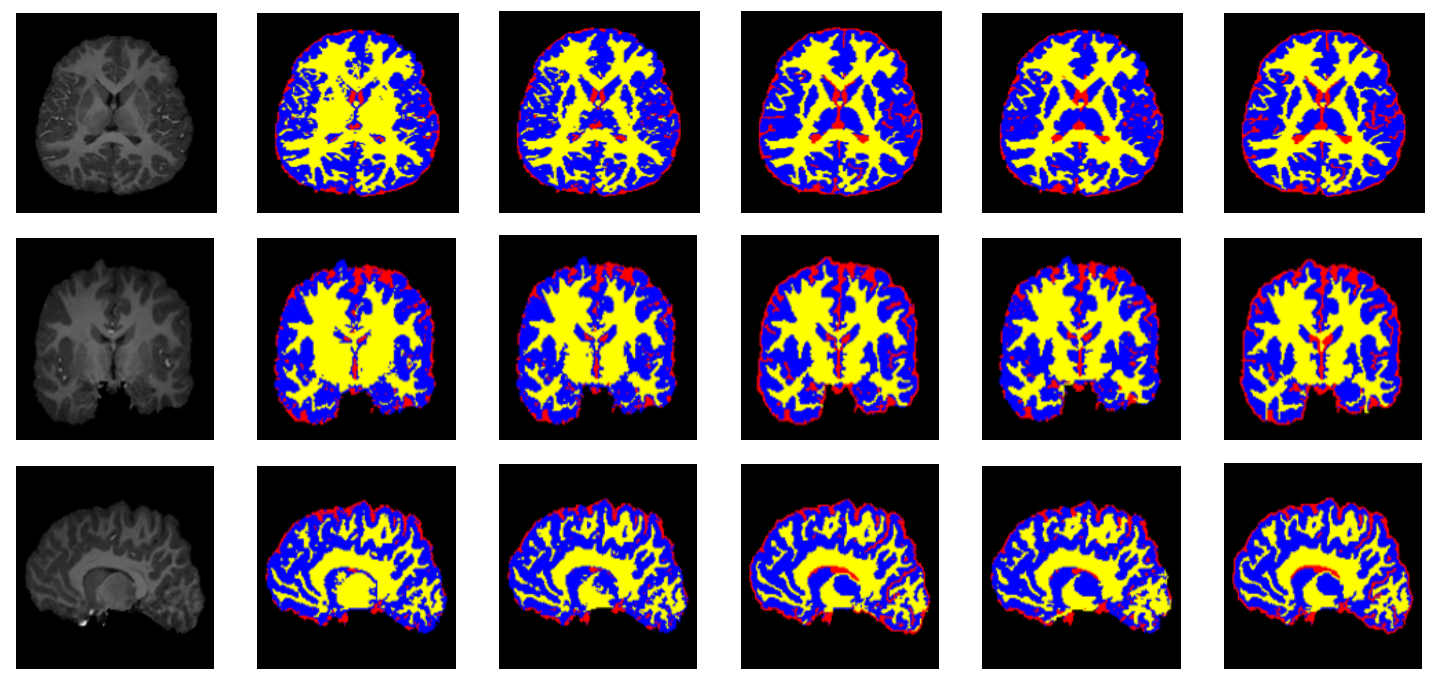

(b)

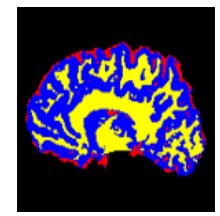

(c)

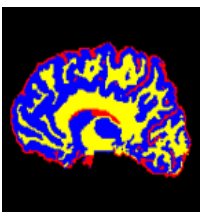

(d)

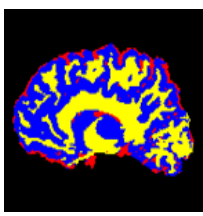

(e)

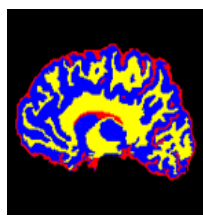

(f)

FIGURE 15: Segmentation results projected onto axial, coronal, and sagittal planes for a sample from the KKI database: (a) original MRIs after skull stripping, and the segmentation using (b) intensity model alone; (c) intensity and shape models (d) intensity, shape, and higher-order MGRF models; and (e) the iBEAT method. Ground truth is shown in (f). CWM is in yellow, GM in blue, and cerebrospinal fluid (CSF) is in red for visualization purposes. All subjects are bias-corrected prior to segmentation using iBEAT or the proposed method.

and validated versus the ground truth images created with the aid of an MR expert using different metrics. The proposed framework was also compared to one of the state-of-the-art approaches and showed better performance. Segmentation is considered to be a key step for the CAD system that will be illustrated in the next chapter for autism diagnosis. If structures of the brain are missing from the segmentation step, then classification decision (autism/control) that comes from the CAD system would not be reliable enough. 
A
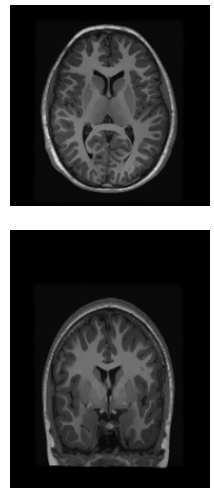

$\mathrm{C}$

$S$

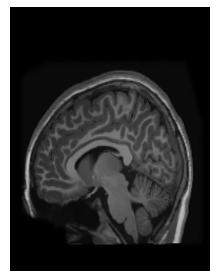

(a)
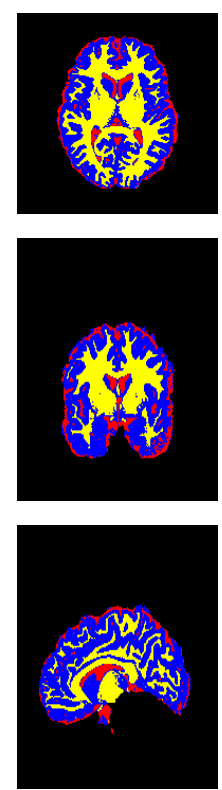

(b)
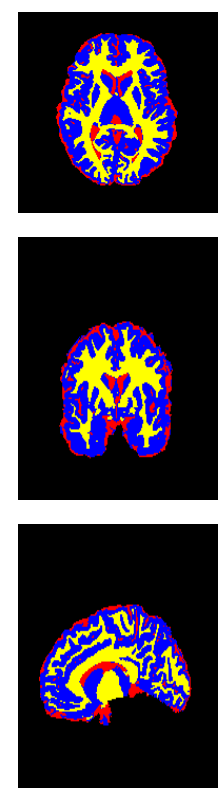

(c)
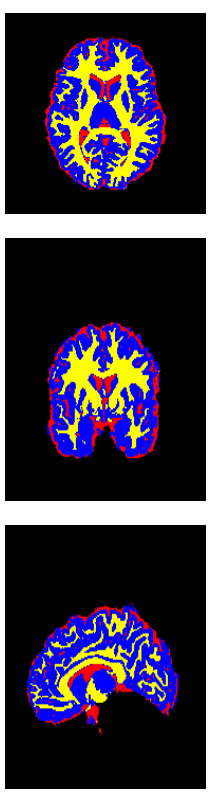

(d)
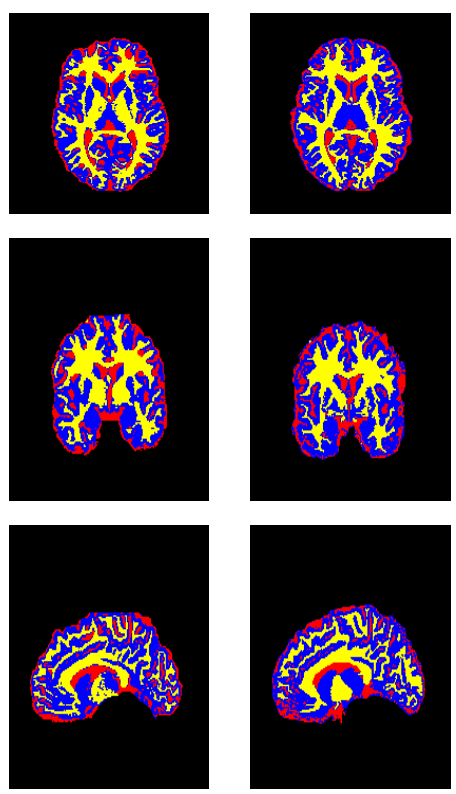

(e)

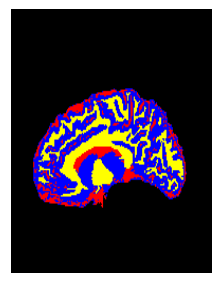

(f)

FIGURE 16: Segmentation results projected onto axial, coronal, and sagittal planes for a sample from the UCLA database: (a) original MRIs after skull stripping, and the segmentation using (b) intensity model alone; (c) intensity and shape models (d) intensity, shape, and higher-order MGRF models; and (e) the iBEAT method. Ground truth is shown in (f). CWM is in yellow, GM in blue, and cerebrospinal fluid (CSF) is in red for visualization purposes. All subjects are bias-corrected prior to segmentation using iBEAT or the proposed method. 


\section{Algorithm 1 Steps of the Proposed segmentation framework}

\section{- MRI Preprocessing and Shape Database Construction}

(a) Use the automated approach in $[326,327]$ to remove the skull from the MR images.

(b) Construct the shape database through a co-alignment of the biased-corrected training brains (both grey scale and their ground truth).

(c) Divide the database into 3 atlases (5 subjects each) using normalized-cross correlation (NCC).

\section{$\circ$ Brain Segmentation}

(a) Align the test subject with the shape database to get the $3 \mathrm{D}$ affine matrix $\mathrm{T}$.

\section{(b) For each Atlas:}

i. Estimate the adaptive shape prior probability:

A. Use the matrix $\mathrm{T}$ to transform each voxel to the atlas domain.

B. Initialize a 3D window and search inside it for voxels with corresponding grey level in all training sets with equalized intensities.

C. Increase the window size, if needed, and redo the search until a nonempty result is found. If maximum size is reached and no result is found, increase the grey level tolerance and get back to Step b.

D. Create probabilities based on the labels from the search results.

ii. Approximate $P(\mathrm{~g})$ using an LCDG with four dominant modes.

iii. Form region map $\mathbf{m}$ using marginal estimated density and prior shape.

iv. Find the Gibbs potentials for the MGRF model from the initial map m.

v. Improve $\mathbf{m}$ using the iterative conditional mode (ICM) algorithm [334].

(c) Apply majority voting to fuse the segmentation results of the three atlases. 
A
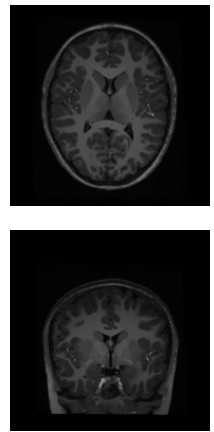

$\mathrm{S}$

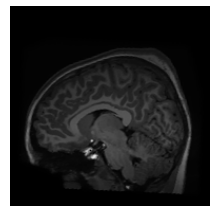

(a)
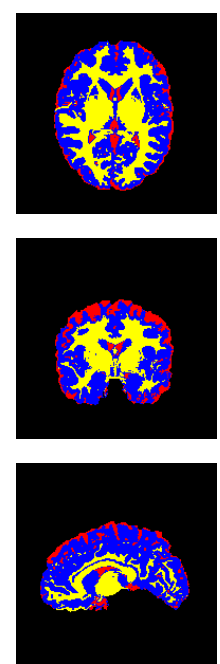

(b)
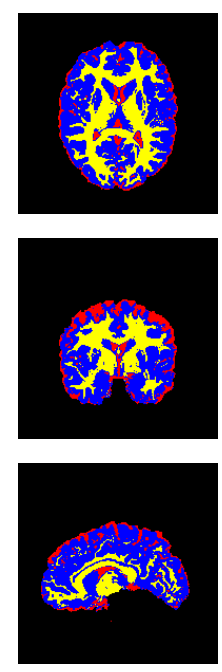

(c)
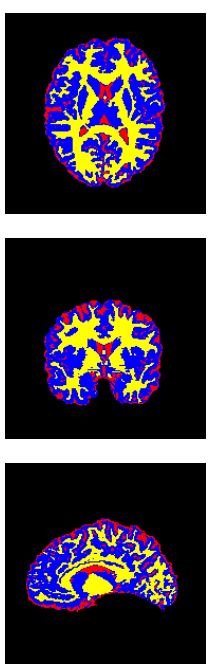

(d)
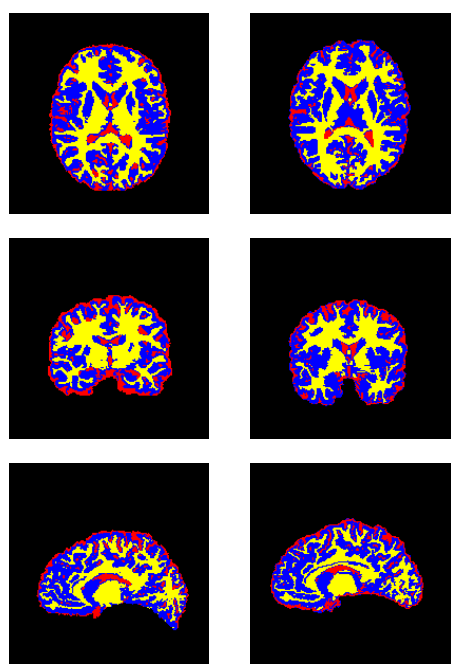

(e)
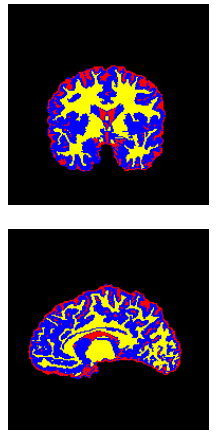

(f)

FIGURE 17: Segmentation results projected onto axial, coronal, and sagittal planes for a sample from the NYU database: (a) original MRIs after skull stripping, and the segmentation using (b) intensity model alone; (c) intensity and shape models (d) intensity, shape, and higher-order MGRF models; and (e) the iBEAT method. Ground truth is shown in (f). CWM is in yellow, GM in blue, and cerebrospinal fluid (CSF) is in red for visualization purposes. All subjects are bias-corrected prior to segmentation using iBEAT or the proposed method. 
TABLE 1: Accuracy of the proposed segmentation approach using Dice Similarity Coefficient (DSC)(\%), the modified Hausdorff Distance (MHD), and Absolute Brain Volume Difference (ABVD) (\%) for the IBIS database. Metrics are represented as Mean \pm Standard Deviation.

\begin{tabular}{|l|c|c|c|c|}
\cline { 2 - 5 } \multicolumn{1}{c|}{} & \multicolumn{2}{c|}{ WM } & \multicolumn{2}{c|}{ CGM } \\
\hline Metric & Ours & iBEAT & Ours & iBEAT \\
\hline DSC & $94.7 \pm 1.53$ & $73.3 \pm 1.27$ & $93.86 \pm 0.13$ & $81.6 \pm 3.5$ \\
\hline$p$-value & & 0.0001 & & 0.0001 \\
\hline \hline MHD & $7.3 \pm 1.23$ & $18.27 \pm 1.53$ & $3.5 \pm 0.24$ & $23.3 \pm 0.52$ \\
\hline$p$-value & & 0.0001 & & 0.0001 \\
\hline \hline ABVD & $3.17 \pm 1.73$ & $37.94 \pm 0.61$ & $1.62 \pm 1.24$ & $34.46 \pm 0.18$ \\
\hline$p$-value & & 0.0001 & & 0.0001 \\
\hline
\end{tabular}

TABLE 2: Accuracy of the proposed segmentation approach using Dice Similarity Coefficient DSC(\%), the modified Hausdorff Distance (MHD), and Absolute Brain Volume Difference $\operatorname{ABVD}(\%)$ for the KKI database. Metrics are represented as Mean \pm Standard Deviation.

\begin{tabular}{|l|c|c|c|c|}
\cline { 2 - 5 } \multicolumn{1}{c|}{} & \multicolumn{2}{c|}{ WM } & \multicolumn{2}{c|}{ CGM } \\
\hline Metric & Ours & iBEAT & Ours & iBEAT \\
\hline DSC & $95.8 \pm 1.5$ & $85 \pm 3.7$ & $96.7 \pm 1.2$ & $88 \pm 2.7$ \\
\hline$p$-value & & 0.0001 & & 0.0001 \\
\hline \hline MHD & $5.2 \pm 1.5$ & $10.2 \pm 3.4$ & $3.1 \pm 1.7$ & $15.1 \pm 3.5$ \\
\hline$p$-value & & 0.0001 & & 0.0001 \\
\hline \hline ABVD & $2.5 \pm 1.6$ & $23.3 \pm 1.1$ & $1.12 \pm 1.1$ & $18.4 \pm 1.8$ \\
\hline$p$-value & & 0.0001 & & 0.0001 \\
\hline
\end{tabular}


TABLE 3: Accuracy of the proposed segmentation approach using Dice Similarity Coefficient DSC(\%), the modified Hausdorff Distance MHD, and Absolute Brain Volume Difference $\operatorname{ABVD}(\%)$ for the NYU and UCLA databases. Metrics are represented as Mean \pm Standard Deviation.

\begin{tabular}{|l|c|c|c|c|}
\cline { 2 - 5 } \multicolumn{1}{c|}{} & \multicolumn{2}{c|}{ WM } & \multicolumn{2}{c|}{ CGM } \\
\hline Metric & Ours & iBEAT & Ours & iBEAT \\
\hline DSC & $96.1 \pm 1.5$ & $87.3 \pm 1.2$ & $97.8 \pm 0.13$ & $89.6 \pm 2.5$ \\
\hline$p$-value & & 0.0001 & & 0.0001 \\
\hline \hline MHD & $2.3 \pm 1.2$ & $15.6 \pm 1.09$ & $0.9 \pm 0.2$ & $13.1 \pm 1.2$ \\
\hline$p$-value & & 0.0001 & & 0.0001 \\
\hline \hline ABVD & $1.5 \pm 1.7$ & $15.4 \pm 1.1$ & $1.2 \pm 1.4$ & $12.6 \pm 1.8$ \\
\hline$p$-value & & 0.0001 & & 0.0001 \\
\hline
\end{tabular}




\section{CHAPTER III}

\section{A CAD SYSTEM FOR AUTISM DIAGNOSIS USING STRUCTURAL AND FUNCTIONAL MRI MODALITIES}

This chapter introduces a novel shape-based computer-aided diagnosis (CAD) system using magnetic resonance (MR) brain images for autism diagnosis. In order to improve the robustness of classification, the proposed system is based on fusing the shape features extracted from the cerebral cortex $(\mathrm{Cx})$ and cerebral white matter (CWM) from sMRI with findings from fMRI. Extracting shape features from both CWM and $\mathrm{Cx}$ is conducted based on the recent findings suggesting that $\mathrm{Cx}$ changes in autism are related to CWM abnormalities. Adding fMRI modality is for the sake of exploiting different modalities in order to better assess ASD.

The CAD system starts with segmenting $\mathrm{Cx}$ and CWM using a 3D joint model that combines intensity, shape, and spatial information. Then, to accurately extract the 3D shape features of Cx, Spherical Harmonic (SPHARM) is applied to its re-constructed meshes, from which four metrics are derived for each mesh point; normal curvature, mean curvature, gaussian curvature, and $\mathrm{Cx}$ surface reconstruction error. To analyze the CWM shape, its gyri are extracted by propagating an orthogonal wave to their surfaces using 3D fast marching and then their distance maps are computed as a shape feature. In addition, curvature analysis is estimated on CWM gyri surfaces using three shape features (curvedness, sharpness, and mean curvature).

Next, a monetary reward fMRI experiment that monitors areas of activation in the brains of both groups in response to the task is conducted. Findings from both experiments are then correlated to local areas of the brain in order to integrate the two modalities for better assessment of ASD and obtain better classification results. This is done by feeding 
all the extracted features to a multi-level deep network for feature fusion and diagnosis.

In this work, all features are extracted from local areas of the brain, where the brains of subjects included in the study are parcellated into 34 cortical regions per hemisphere. This atlas is known as Desikan-Killiany (DK) labeling atlas [340]. This local analysis would allow for better understanding of ASD and linking this complex syndrome to different functional and behavioral activities of the brain.

Results on the subjects included in this study (22 with ASD, 25 controls) showed high classification accuracy between the two groups. First, the classification was computed based on CWM features alone, and the accuracy achieved was $81 \%$. The classification accuracy obtained using $\mathrm{Cx}$ features alone was $76 \%$. Integrating features from both structures generated a classification accuracy of $85.11 \%$. This comes in line with the previous neuropathological studies suggesting that the abnormalities in CWM connections are related to $\mathrm{Cx}$ changes in ASD.

Next, the features obtained from the applied monetary reward fMRI experiment were used for classification, generating an accuracy of $89.4 \%$. Finally, fusing all features form all modalities generated a classification accuracy of $94.74 \%$, which shows the merit of integrating multiple modalities for ASD diagnosis.

\section{A. Introduction}

The term "autism spectrum disorder" (ASD) refers to a collection of neuro-developmental disorders that affect linguistic, behavioral, and social skills. Autism has many symptoms, most prominently, social impairment and repetitive behaviors. The most severe form of the ASDs is autistic disorder (AD). By some estimates, ASD affects 1 out of 68 eight years of age, with males being four times more likely to develop it than females. Autism is typically diagnosed at the age of 3; however, some characteristics can sometimes be observed at around 12 months of age. What causes autism is yet unknown; however, it is mainly believed that genetic and environmental factors in complex combinations are responsible [1]. 
In the literature, several imaging modalities have investigated the changes associated with autism such as structural MRI (sMRI), diffusion tensor imaging (DTI), and functional MRI (fMRI). sMRI examines anatomical abnormalities of the brain's structures such as Cx, CWM, and hippocampus, whereas DTI characterizes the 3D diffusion of brain water molecules, hence helping elucidate abnormal white matter connectivity in developmental disorders. fMRI measures the abnormalities through monitoring changes with the brain's blood flow.

It is worth mentioning that many acquisition sites in the US have been collecting sMRI data for a long time, leading to the establishment of comprehensive databases. Examples are NDAR/IBIS, NDAR/Pitt [336], and ABIDE [335], with some sites collecting data using other modalities such as DTI data [336]. This work focuses on both sMRI and fMRI data, having a long-term plan of integrating data from other modalities. sMRI studies on ASD can be categorized into either ROI-based volumetry (RBV), or shape-based morphometry (SBM). RBV approaches focus on the total volume for a particular region, yet suffer from being age and gender-sensitive and in need of age-correction coefficients, which does not enable having robust CAD systems.

On the other hand, SBM studies mainly address topological shape features that cannot be directly estimated using RBV, such as surface curvature and cortical thickness. SBM approaches are capable of accounting for the brain's inherent topological features, however most of the work at present work directly on the meshes generated from raw data without parameterizing the brain or aligning the meshes, which makes the computations sensitive to pre-processing and hinders comparing information. Please refer to Chapter 2 for a comprehensive survey on fMRI and SMRI in ASD.

To overcome these limitations, this work proposes a novel automated approach for autism diagnosis using shape features from sMRI as well as findings from fMRI. The motivation behind fusing CWM and Cx features from sMRI is that recent studies suggest that neuropathological findings in regards to a putative minicolumnopathy and dysplastic 
Cx changes tend to magnify themselves by abnormal connections of CWM [274]. Also, it is thought that integrating different imaging modalities such as sMRI and fMRI would improve the diagnosis accuracy, and that was the motivation behind conducting an fMRI experiment and fusing its results with those of the sMRI experiment.

\section{B. Methods}

The proposed CAD system starts with the segmentation of the brain MR images into CWM and Cx. The sMRI experiment is then conducted, which performs shape analysis on the reconstructed meshes of $\mathrm{Cx}$ and CWM, from which 8 shape features are extracted. A monetary reward fMRI experiment is then applied that finds areas of activation of the brain that are related to that specific task. Features were calculated per anatomical area of the brain to provide local analysis, using two labeling atlases; DK atlas, Figure 19, which divides the brain into 34 cortical regions per hemisphere (total number of shape features is 544 (8 per region)). All features from both experiments are finally fused to classify autistic and control brains. The steps of the proposed CAD system are illustrated in Figure. 18.

\section{Segmentation of CWM and Cx from MR images}

Segmentation of the MR brain images is conducted using the proposed framework in Chapter 3, which is based on a 3D joint model that integrates shape, intensity, and spatial information. Details of the proposed segmentation framework are in Chapter 3.

\section{SMRI experiment: shape feature extraction}

After segmenting $\mathrm{Cx}$ and $\mathrm{CWM}$, shape analysis is conducted to obtain the CAD

system. Four shape features have been extracted from 68 local areas of the brain's Cx (34 areas per hemisphere). Four other shape features from CWM have also been extracted, resulting in a total number of 544 features per scan. Shape features details are outlined 

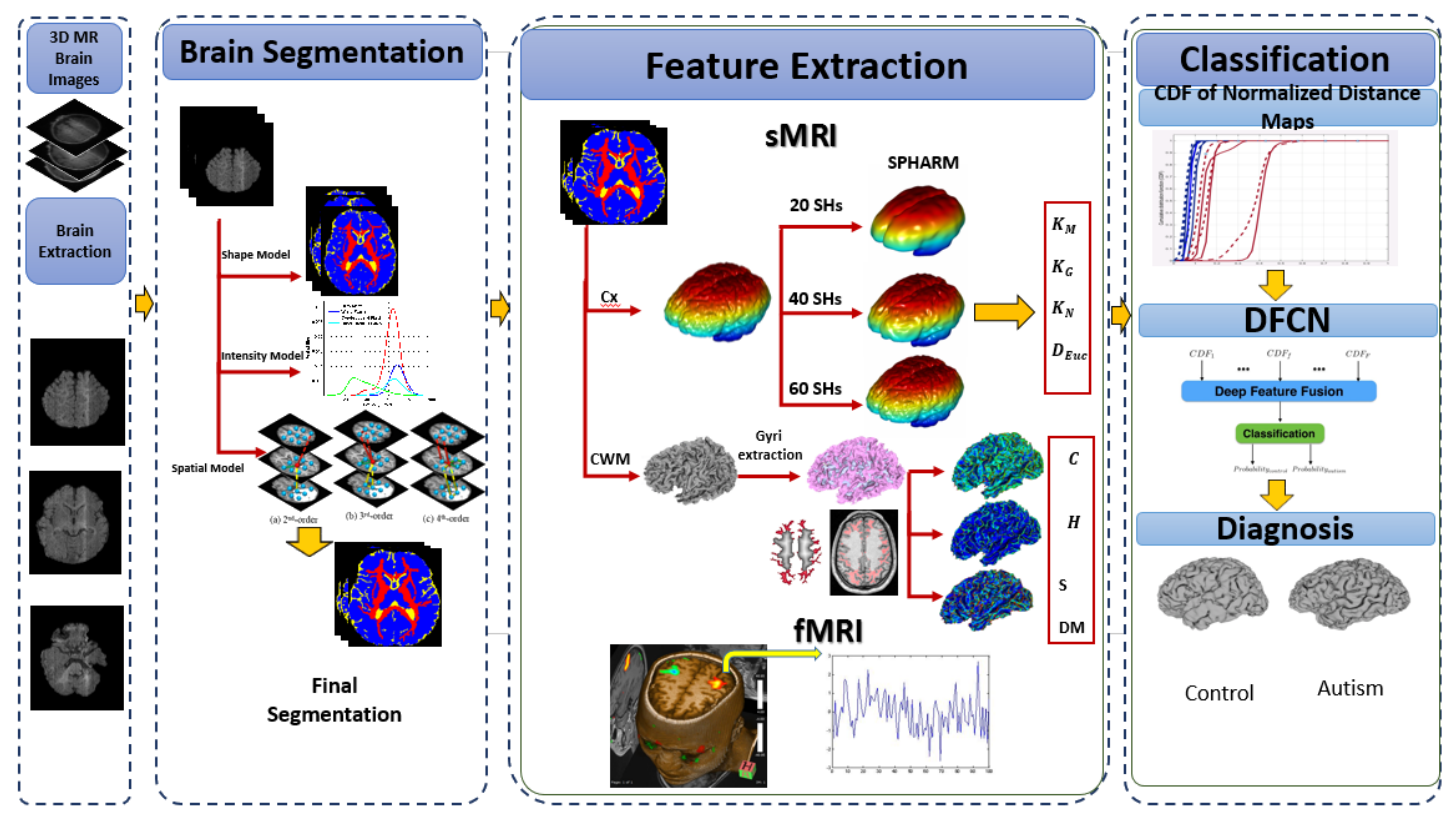

FIGURE 18: Illustration of the proposed CAD system.

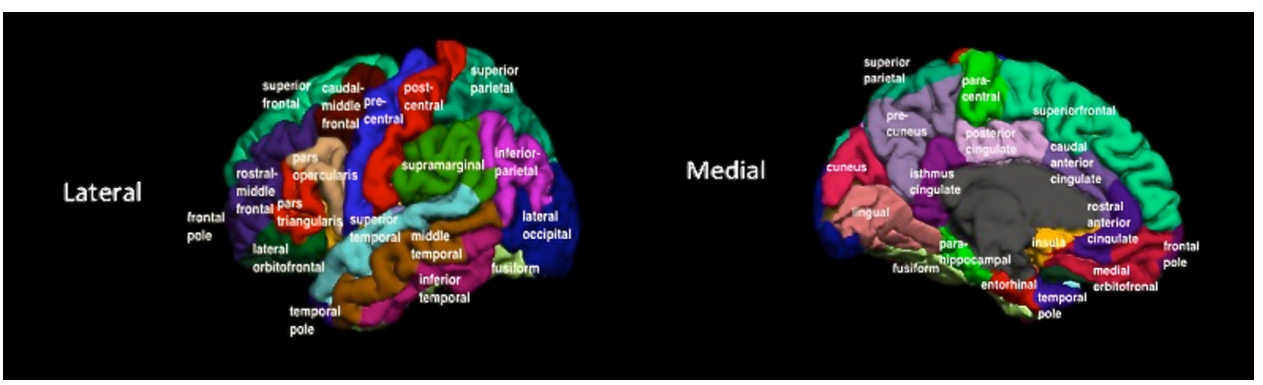

FIGURE 19: Desikan-Killiany Labeling Atlas

below.

a. Cx shape features : to extract the $\mathrm{Cx}$ shape features, accurate approximation of its 3D shape is required as well as having the ability to compare different brain subjects. One way to achieve so is SPHARM analysis [341,342] that approximates the cortex surface so that the sensitivity to any errors that might have resulted from the segmentation is greatly reduced. It also makes the reconstruction insensitive to data acquisition from different sites. In order to apply SPHARM, a mesh manifold is first generated from the segmented scans using a modified version of the TETGEN algorithm [343]. When con- 
structing the meshes, there is a restriction on the number of nodes to be always the same for all subjects. This is because the shape of each mesh differs from the other, while there must be a consistent number of nodes present so that meshes can be aligned with one another. Alignment is done using SPAHRM-based registration [342]. Each node of the mesh is then characterized with 4 features. These metrics are: Cx reconstruction error, by computing the Euclidean distance from the origin $D_{\text {Euc }}$; Gaussian curvature $K_{G}=\mathbf{K} 1 \mathbf{K} \mathbf{2}$ (product of the principal curvatures); mean curvature $K_{M}=(\mathbf{K} \mathbf{1}+\mathbf{K} 2) / 2$ (the average of the principal curvatures); and normal surface curvature $K_{N}=\max (\mathbf{K} \mathbf{1}, \mathbf{K 2})$ (maximum of the two principal curvatures).

b. CWM shape features : in order to extract CWM gyri, the distance map is calculated inside the segmented 3D CWM by a fast marching level set method [344], which gives the minimum Euclidean distance from each inner point of the segmented CWM to its boundary. Using the EM-based approach in [345], the mixed empirical marginal distribution of these distances is partitioned into two probability models: of the CWM gyri (class 1) and all other CWM tissues (class 2), respectively. Then the gyri are extracted using the optimum threshold that separates the two classes.

First, a 3D fast marching level-set method is used to propagate a wave front in order to find voxels located at a distance less than or equal to $\mathbf{T}$ from the boundary of CWM, Figure 20(b). Another wave is then propagated in order to find voxels located at a distance less than or equal to $\mathbf{T}$ from voxels located at a distance $\mathbf{T}$ from the boundary of CWM, Figure 20(c). Finally, voxels that are visited by the second wave are removed from the ones visited by the first wave. The remaining part represents the extracted CWM gyri, Figure 20(d), (e).

After extracting gyri from CWM, labeling of the brain regions was done using FreeSurfer software [340], where an automated labeling system for subdividing the human cerebral cortex on MRI scans into 34 gyral based regions of interest is applied. This would allow for local analysis of the different brain anatomical areas, which would subsequently 


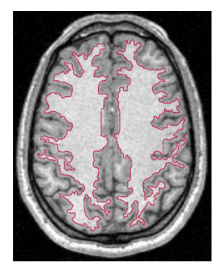

(a)

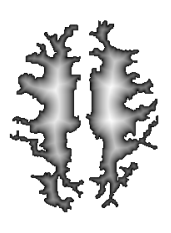

(b)

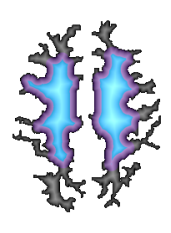

(c)

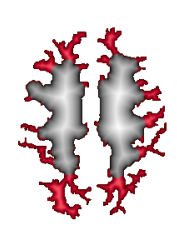

(d)

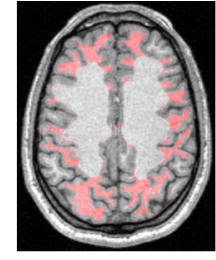

(e)

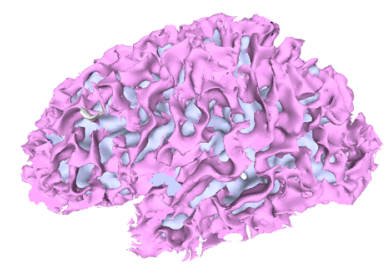

(f)

FIGURE 20: (a) Boundary of segmented CWM. (b) First distance map. (c) Second distance map. (d), (e) Extracted CWM gyri. (f) 3D visualization of the extracted gyri.

aid in correlating ASD with different functional and behavioral activities.

From the extracted gyral CWM, the distance maps, $D M$, were calculated in order to be used as a shape feature for gyral CWM. In addition, curvature-based analysis is conducted for the 3D brain CWM meshes. Three features are calculated: the mean curvature

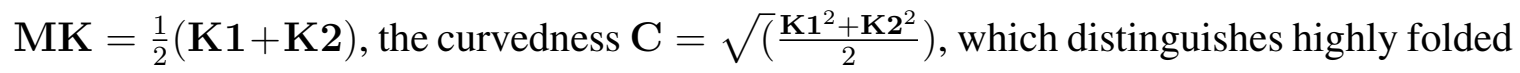
regions from less folded ones [83], and the sharpness $\mathbf{S}=\left((\mathbf{K} \mathbf{1}-\mathbf{K} 2)^{2}\right)$, which is used to quantify the sharpness of folding [83].

\section{FMRI experiment: monetary reward feedback}

This study is based on the experiment described in Scott-Van Zeeland et al. [346], where two categories of images were displayed to the participants who were asked to press a button to classify those images, and subsequently, a monetary feedback was provided. In this work, the monetary rewards data of 19 subjects (8 ASD, and 11 controls) were used. Each trial in the experiment is 5 seconds, where the images are displayed for approximately 2 seconds, the feedback is displayed for 1.25 seconds, and finally a rest period of approximately 1.75 seconds occurs between trials [346]. Each volume was preprocessed through four steps: i) slice time correction to overcome the effect of capturing 2D slices at different times through the whole volume, ii) realignment of slices to overcome motion artifacts. The realignment uses six parameters, spatial transformation, and least squares approach, 
iii) normalization of slices to fit them into standard ICBM 152 template space [347] with voxel size of 2x2x2 mm, and finally iv) smoothing using a Gaussian kernel. In this experiment, the BOLD activation change during the feedback display was modeled using GLM with one regressor describing the feedback event timing and a first group analysis was conducted using SPM12 package [348]. For every subject, voxels that exhibited the most significant BOLD signal activation change due to the monetary reward feedback event are extracted and sorted. At each significant voxel, 180 values were recorded over the time series and compared to the expected GLM convolved with the HRF as a reference signal, Figure 21. Although both BOLD signal changes and the reference signal are in the time domain, they are both converted first to the frequency domain before comparison. This is done because comparing in frequency domain gives better results due to the abrupt change pattern in the reference signal.

\section{Deep Fusion Classification Network (DFCN)}

The goal of this stage is to obtain a global diagnosis from the collected shape features for each one of the local areas the brain is parcellated into. For the sMRI experiment, the traditional way to achieve so is to use the raw features in a vertex-wise manner, but this is inefficient since the number of nodes for each mesh is $48 \mathrm{~K}$. This will result in having around $400 \mathrm{~K}$ points per subject which is time consuming. To avoid this, features are represented using their cumulative distribution function (CDF) values that would retain all information as well as reduce data dimensionality.

As per the fMRI experiment, the input to the deep network is the vector that comprises the values recorded over the time series for all significant voxels identified in all anatomical areas.

The CDFs are calculated with the minimum increment obtained from sorting all measures and selecting the minimum difference between consecutive points to capture all information in the distribution. Having this huge number of features per subject (544) 
would require a deep network scheme to account for all data. The model is built from a stack of auto-encoders (AEs) and output layer of softmax regression for each feature [349], and is composed of two stages, Figure 22. In the first stage, the CDF for each feature of the 544 is learnt separately with an AE using sparsity (KL-divergence) and non negative constraints, in order to capture most prominent variations and thus obtain discriminatory features. The scheme employed here uses AEs with non-negativity constraint (NCAE) [350] that decompose the data, along with sparsity (KL divergence), to extract the most uncorrelated and definitive features based on non-negative matrix factorization. Second stage is a supervised back-propagation method that minimizes the total loss (negative log-likelihood) for given training labeled data. Finally, the high-level features extracted from each stacked NCAE (SNCAE) are concatenated, as a new combined high-level feature, and fed into another SNCAE for non-linear fusion and final diagnosis, by taking two probabilities (autism, control) in its output layer. This is where the term "deep fusion" comes from.

Let $\mathbf{W}=\left\{\mathbf{W}_{j}^{\mathrm{e}}, \mathbf{W}_{i}^{\mathrm{d}}: j=1, \ldots, s ; i=1, \ldots, n\right\}$ denote a set of column vectors of weights for encoding (e) and decoding (d) layers of a single AE . Let T denote vector transposition. The AE converts an $n$-dimensional column vector $\mathbf{u}=\left[u_{1}, \ldots, u_{n}\right]^{\top}$ of input signals into an $s$-dimensional column vector $\mathbf{h}=\left[h_{1}, \ldots, h_{s}\right]^{\top}$ of hidden codes (features, or activations), such that $s \ll n$, by uniform nonlinear transformation of $s$ weighted linear combinations of signals:

$$
h_{j}=\sigma\left(\left(\mathbf{W}_{j}^{\mathrm{e}}\right)^{\top} \mathbf{u}\right) \equiv \sigma\left(\sum_{i=1}^{n} w_{j: i}^{\mathrm{e}} u_{i}\right)
$$

where $\sigma(\ldots)$ is a certain sigmoid, i.e., a differentiable monotone scalar function with values in the range $[0,1]$. Unsupervised pre-training of the AE minimizes total deviations between each given training input vector $\mathbf{u}_{k} ; k=1, \ldots, K$, and the same-dimensional vector, $\widehat{\mathbf{u}}_{\mathbf{W}: k}$ reconstructed from its code, or activation vector, $\mathbf{h}_{k}$. The total reconstruction error of applying such $\mathrm{AE}$ to compress and decompress the $K$ training input vectors integrates the 
$\ell_{2}$-norms of the deviations:

$$
J_{\mathrm{AE}}(\mathbf{W})=\frac{1}{2 K} \sum_{k=1}^{K}\left\|\widehat{\mathbf{u}}_{\mathbf{W}: k}-\mathbf{u}_{k}\right\|^{2}
$$

To reduce the number of negative weights and enforce sparsity of the NCAE, the reconstruction error of Equation (12) is appended, respectively, with quadratic negative weight penalties, $f\left(w_{i}\right)=\left(\min \left\{0, w_{i}\right\}\right)^{2} ; i=1, \ldots, n$, and Kullback-Leibler (KL) divergence, $J_{\mathrm{KL}}\left(\mathbf{h}_{\mathbf{W}^{e}} ; \gamma\right)$, of activations, $\mathbf{h}_{\mathbf{W}^{\mathrm{e}}}$, obtained with the encoding weights $\mathbf{W}^{\mathrm{e}}$ for the training data, from a fixed small positive average value, $\gamma$, near 0 :

$$
J_{\mathrm{NCAE}}(\mathbf{W})=J_{\mathrm{AE}}(\mathbf{W})+\alpha \sum_{j=1}^{s} \sum_{i=1}^{n} f\left(w_{j: i}\right)+\beta J_{\mathrm{KL}}\left(\mathbf{h}_{\mathbf{W}^{\mathrm{e}}} ; \gamma\right)
$$

Here, the factors $\alpha \geq 0$ and $\beta \geq 0$ specify relative contributions of the non-negativity and sparsity constraints to the overall loss, $J_{\mathrm{NCAE}}(\mathbf{W})$, and

$$
J_{\mathrm{KL}}\left(\mathbf{h}_{\mathbf{W}^{\mathrm{e}}}, \gamma\right)=\sum_{j=1}^{s} h_{\mathbf{W}^{\mathrm{e}: j}} \log \left(\frac{h_{\mathbf{W}^{\mathrm{e}}: j}}{\gamma}\right)+\left(1-h_{\mathbf{W}^{\mathrm{e}: j}}\right) \log \left(\frac{1-h_{\mathbf{W}^{\mathrm{e}: j}}}{1-\gamma}\right)
$$

The classifier is built by stacking the NCAE layers with an output softmax layer. Each NCAE is pre-trained separately in the unsupervised mode, by using the activation vector of a lower layer as the input to the upper layer. In our case, the initial input data consisted of the 100-component CDFs, each of size 100. The bottom NCAE compresses the input vector to $s_{1}=50$ first-level activators, compressed by the next NCAE to $s_{2}=5$ second-level activators, which are reduced in turn by the output softmax layer to $s^{\circ}=2$ values.

Separate pre-training of the first and second layers by minimizing the loss of Equation (13) reduces the total reconstruction error, as well as increases sparsity of the extracted activations and numbers of the non-negative weights. The activations of the second NCAE layer, $\mathbf{h}^{[2]}=\sigma\left(\mathbf{W}_{[2]}^{\mathrm{e}}{ }^{\top} \mathbf{h}^{[1]}\right)$, are inputs of the softmax classification layer to compute a plausibility of a decision in favor of each particular output class, $c=1,2$ :

$$
p\left(c ; \mathbf{W}_{\circ: c}\right)=\frac{\exp \left(\mathbf{W}_{\circ: c}^{\top} \mathbf{h}^{[2]}\right)}{\exp \left(\mathbf{W}_{\circ: 1}^{\top} \mathbf{h}^{[2]}\right)+\exp \left(\mathbf{W}_{\circ: 2}^{\top} \mathbf{h}^{[2]}\right)} ; c=1,2 ; \sum_{c=1}^{2} p\left(c ; \mathbf{W}_{\circ: c} ; \mathbf{h}^{[2]}\right)=1 .
$$


Its separate pre-training minimizes the total negative log-likelihood $J_{\circ}\left(\mathbf{W}_{\circ}\right)$ of the known training classes, appended with the negative weight penalties:

$$
J_{\circ}\left(\mathbf{W}^{o}\right)=-\frac{1}{K} \sum_{k=1}^{K} \log p\left(c_{k} ; \mathbf{W}_{\circ: c}\right)+\alpha \sum_{c=1}^{2} \sum_{j=1}^{s_{2}} w_{\circ: c: j}
$$

Finally, the entire stacked NCAE classifier (SNCAE) is fine-tuned on the labeled training data by the conventional error back-propagation through the network and penalizing only the negative weights of the softmax layer. In these experiments, $\alpha=0.03, \beta=3$, and $\gamma=0.1$.

Next, in order to prepare the fMRI input, there must be an NCAE for each anatomical region to provide local analysis of the brain. For each subject, each voxel found to be significant using SPM package was assigned to an anatomical area of the 68. If more than one voxel was found to be significant at the same anatomical region, the one with the smallest p-value was picked for that region. The input vector to the NCAE for that region would be the 180 points recorded over the time series for that voxel. If no significant voxel was found at a specific anatomical region, then the input vecotr to the NCAE for this region would be all zeros.

\section{Experimental Results}

Forty seven subjects (22 autistic (20 Males, 2 Females), 25 controls (all Males)) have been used for this experiment, that have been downloaded from National Database for Autism Research (NDAR). The range of ages for participants was (23 - 210 months) during the time of the experiment, and the range of IQs is (84-118) for autistic individuals.

For the fMRI experiment, a subset from the 47 subjects ( 8 autistic and 11 controls) has been used. T2-weighted echo-planar images were acquired using a 3T MRI scanner $(\mathrm{TR}=2000 \mathrm{msec}, \mathrm{TE}=30 \mathrm{msec}, \mathrm{FOV}=20 \mathrm{~cm}, 33$ slices, $3.125 \mathrm{~mm}$ in-plane resolution,

$4 \mathrm{~mm}$ thick). For each run of the experiment, 180 functional images that lasted for six minutes covering the whole cerebral volume were acquired. Two volumes were used at the 
onset of each run in order to allow equilibration to steady state, then were excluded from the analysis [346].

In order to test the effect of each feature/modality, the NCAE is first trained and tested for each feature individually. So, for each of the 68 anatomical areas, an SNCAE is trained and tested for each of the CWM features. Then, the 4 CWM features were fed into a DFCN and trained and tested for each area in order to provide local analysis of the brain. Then, in order to obtain a global classification decision, all CWM features from all the regions are fused into a DFCN, which achieved an accuracy of $81 \%$.

The same experiment was conducted for $\mathrm{Cx}$, where local analysis was first conducted by training and testing an NCAE for each one of the $4 \mathrm{Cx}$ features at each anatomical region of the 68 . Then, the $4 \mathrm{Cx}$ features were fused per region in order to improve accuracy. Finally, a global classification accuracy is obtained by fusing all features from all regions, achieving an overall accuracy of $78 \%$.

Next, fusing features from both CWM and Cx was done, which achieved an overall classification accuracy of $85.11 \%$. The improvement in accuracy achieved when fusing features from both regions is in line with the previous neuro-pathological studies that proved correlation between these two structures in ASD. It also shows the merit of combining structures for ASD diagnosis.

Next, classification was conducted using the fMRI experiment. Local analysis for each of the 68 areas was done, then all probabilities were fused from all regions in order to obtain a global diagnosis decision. The accuracy of classification based on only the fMRI experiment was $89.4 \%$.

Finally, fusing features from all modalities achieved a classification accuracy of 94.7\%, which emphasizes the significance of combining structures/modalities for ASD diagnosis.

Figures 23 and 24 show different cases of ASD, where DK areas of the cortices of subjects are color-coded according to the strength of association of each area with ASD. 
The four maps are different, which emphasizes the idea of personalized medicine. Figures 25, 26 show the same for WM meshes of eight different subjects.

Another labeling system is provided in this work which parcellates the brain into BAs. This is done by mapping the DK labeling atlas to BAs using Freesurfer's fsaverage data [351].

\section{Analysis of NCAE weights}

In order to evaluate the performance of the NCAE, experiments are conducted that compare the input to the NCAE, e.g. the CDF values in the sMRI experiment, to the same $\mathrm{CDF}$ vector after being reconstructed, getting the so called NCAE reconstruction error at the selected hidden number of nodes. Figures 27, 28, 29, 30 indicate that the number of hidden nodes chosen to conduct these experiments was optimal, since the reconstruction error is very small.

\section{Summary and Conclusion}

To recapitulate, this work proposed a novel automated approach for autism diagnosis. It started with $3 \mathrm{D}$ brain segmentation into $\mathrm{Cx}$ and $\mathrm{CWM}$ form MR images, followed by shape feature extraction from both. Shape features from both structures were fused based on studies suggesting that neuropathological findings in regards to a putative minicolumnopathy and dysplastic $\mathrm{Cx}$ changes tend to magnify themselves by abnormal connections of CWM. An fMRI experiment was also conducted to capture differences between the two groups. Local areas of the brain were then analyzed based on DK atlas labeling system, and were also linked to BAs in order to find any correlation with autism, using a deep fusion network. This work could pave the pathway for better understanding of the autism spectrum by finding local areas that correlate to the disease. The idea of personalized medicine is emphasized in this work. The proposed CAD system holds the promise to resolve autism 
endophenotypes and help clinicians deliver personalized treatment to individuals affected with this complex syndrome. 


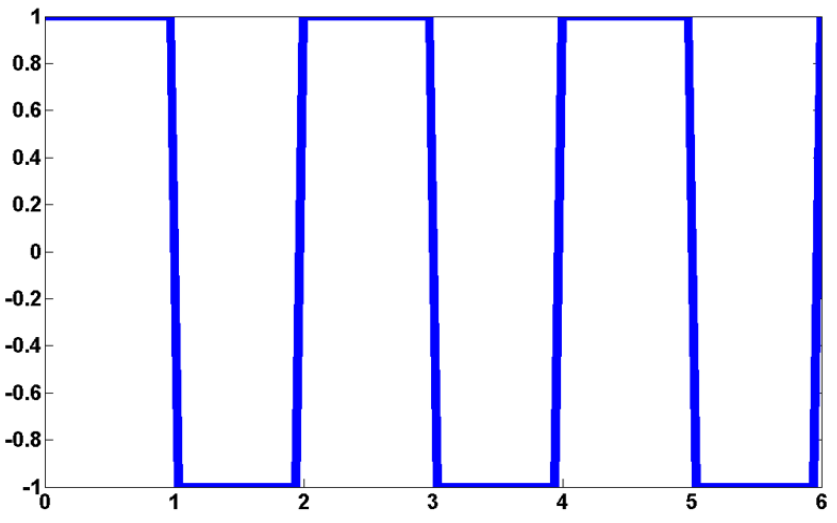

(a)

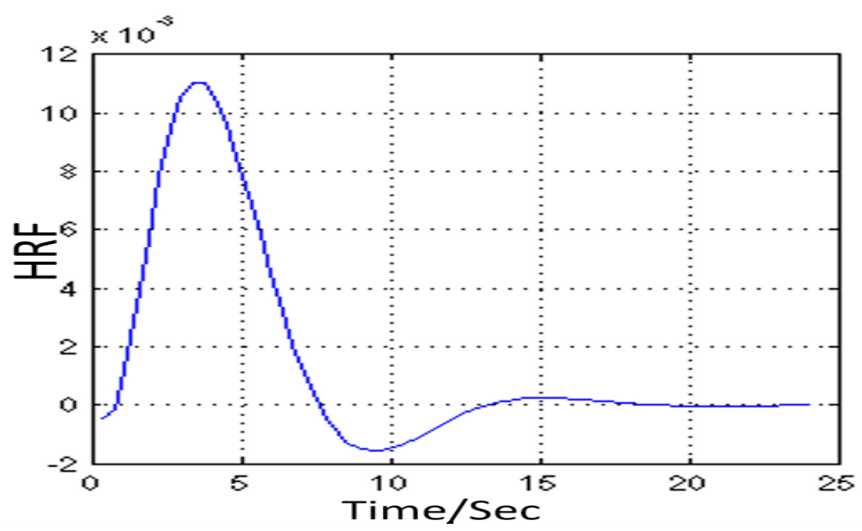

(b)

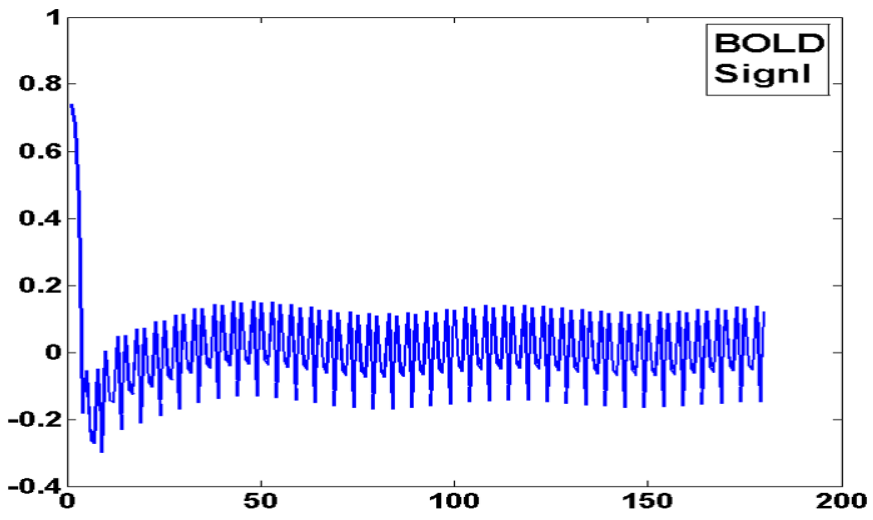

(c)

FIGURE 21: (a) GLM, and (b) the HRF signal. (c) BOLD signal that results from convolving (a) and (b). 


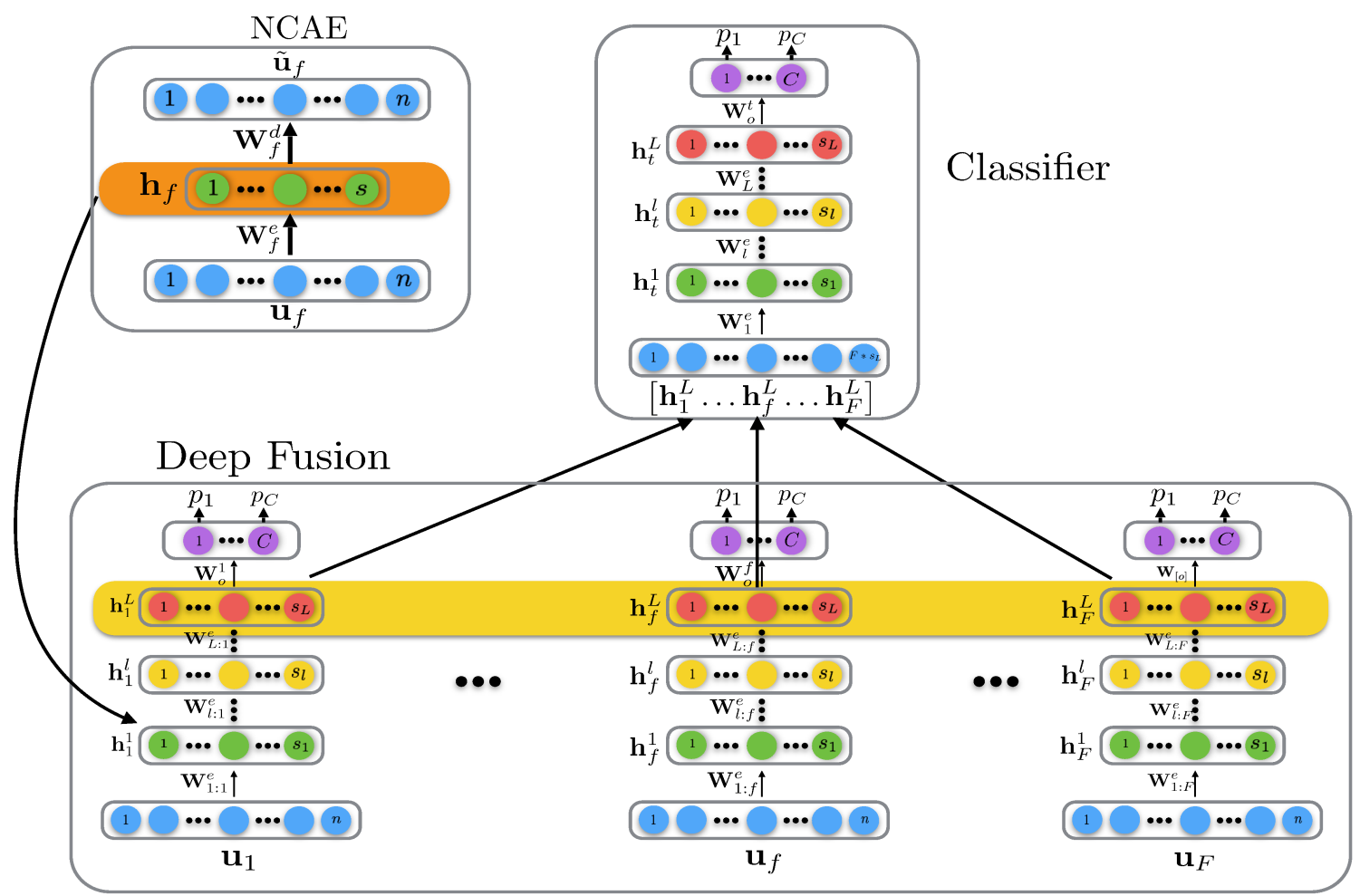

FIGURE 22: Structure of DFCN. 

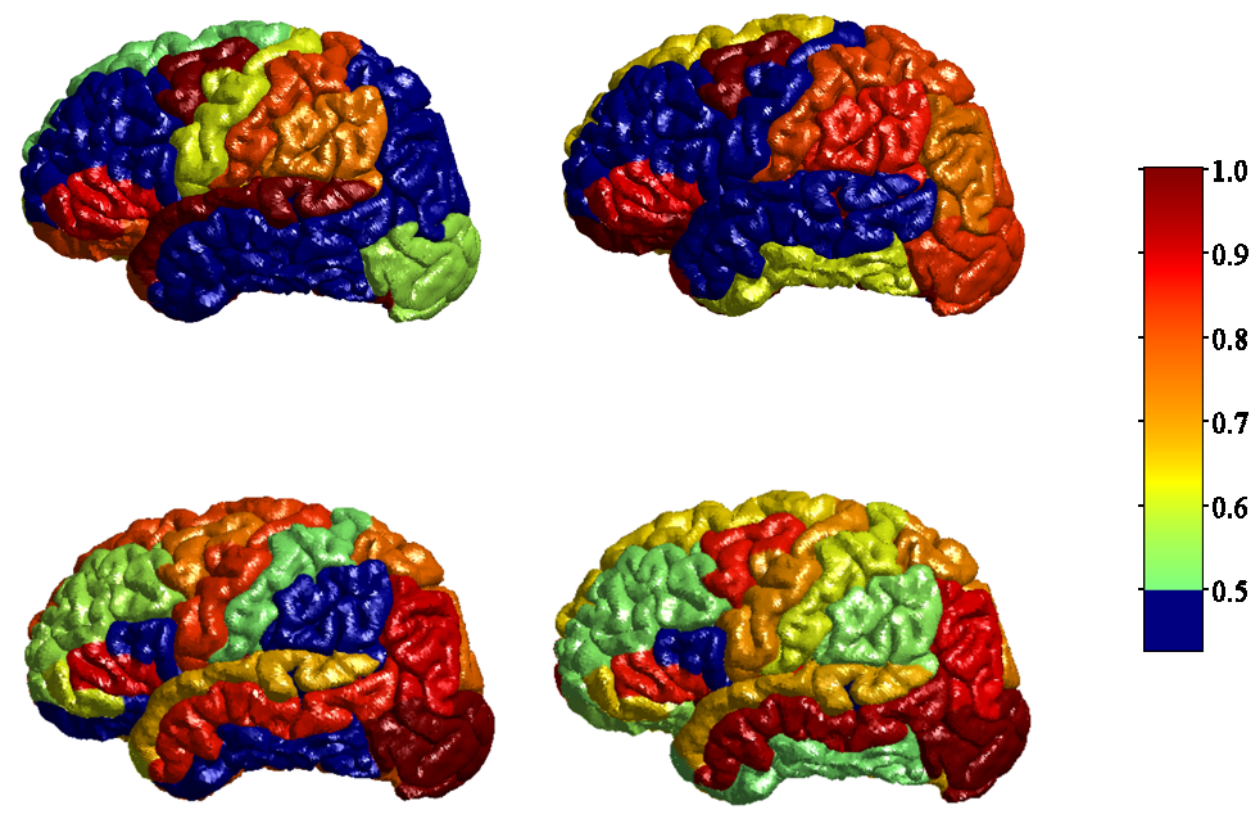

FIGURE 23: Classification of four ASD cases, color coded to indicate the strength of association of each DK area with the ASD phenotype. This emphasizes the significance of personalized medicine.
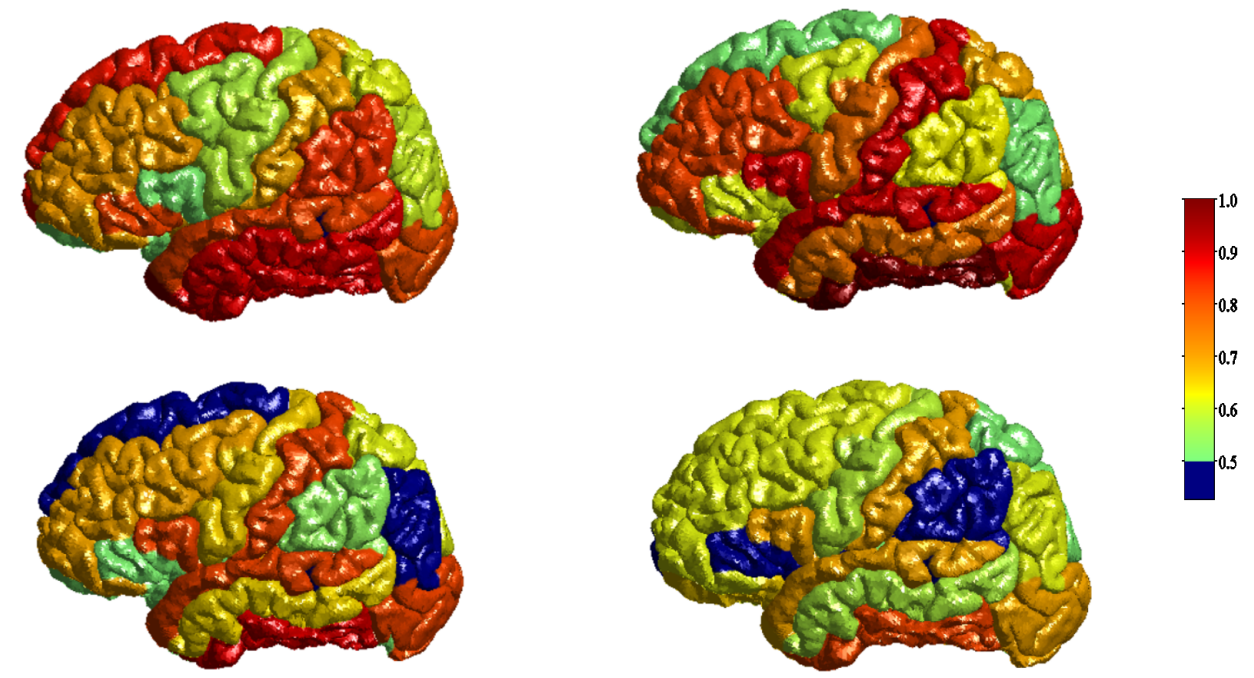

FIGURE 24: Classification of four ASD cases, color coded to indicate the strength of association of each DK area with the ASD phenotype. This emphasizes the significance of personalized medicine. 

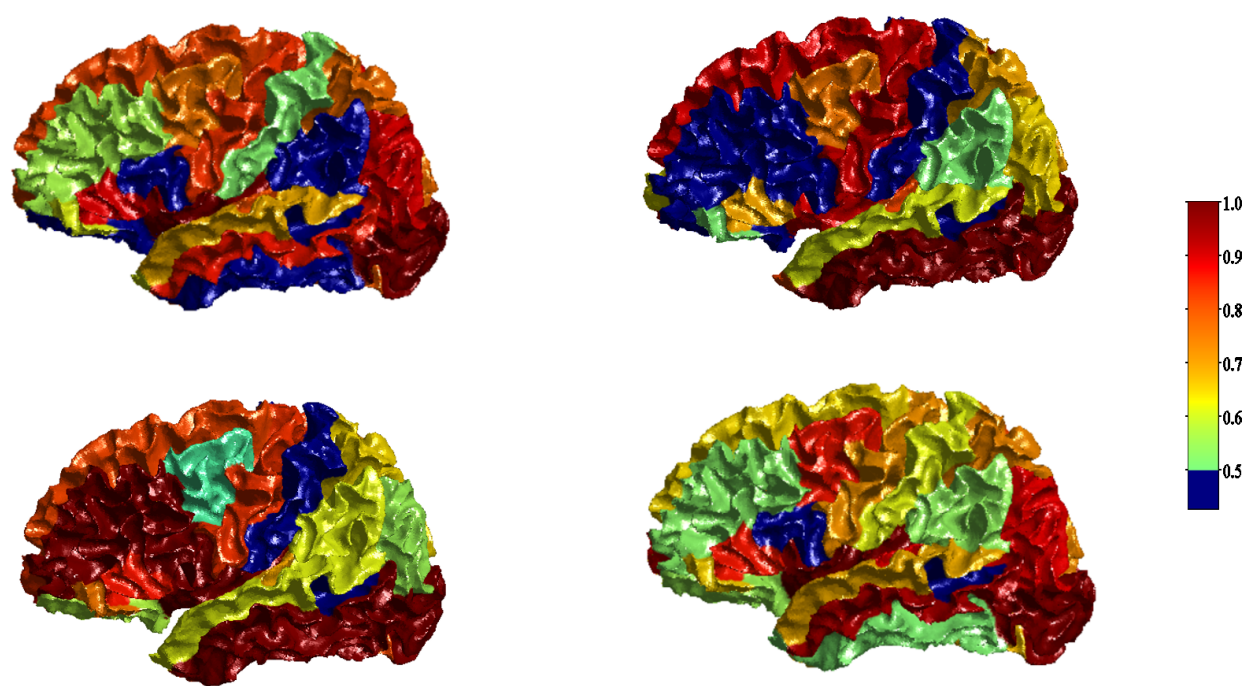

FIGURE 25: Classification of four ASD cases, color coded to indicate the strength of association of each DK area with the ASD phenotype. This emphasizes the significance of personalized medicine.
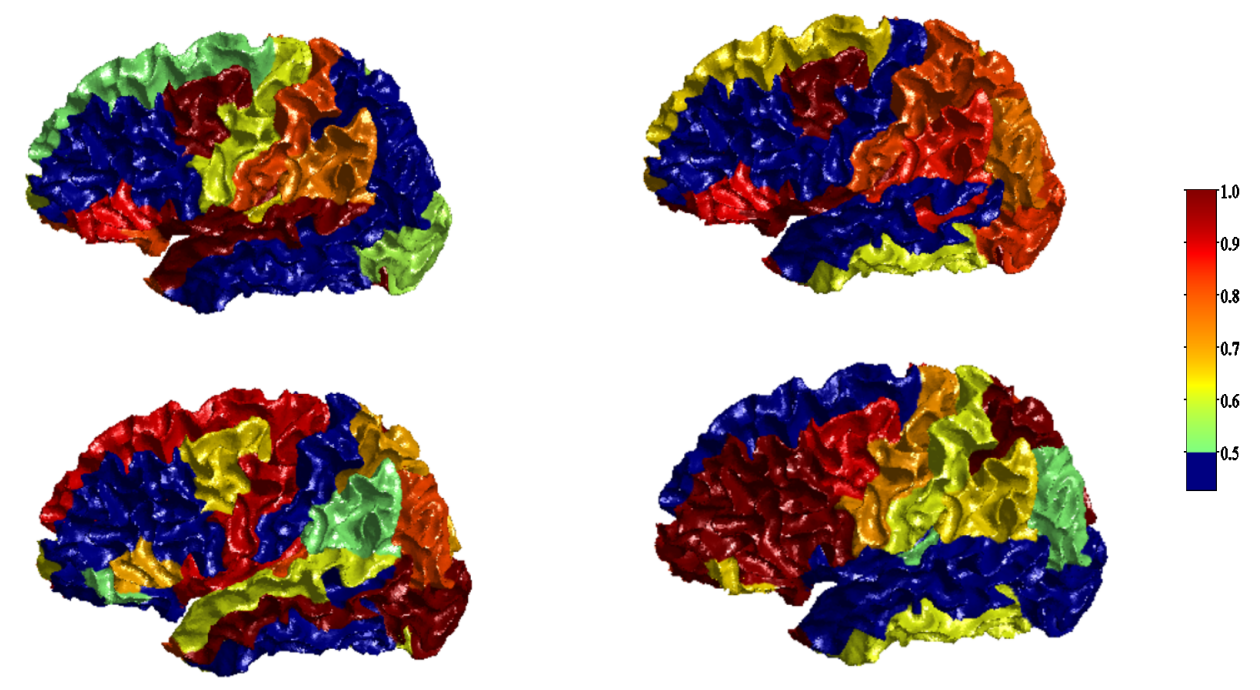

FIGURE 26: Classification of four ASD cases, color coded to indicate the strength of association of each DK area with the ASD phenotype. This emphasizes the significance of personalized medicine. 


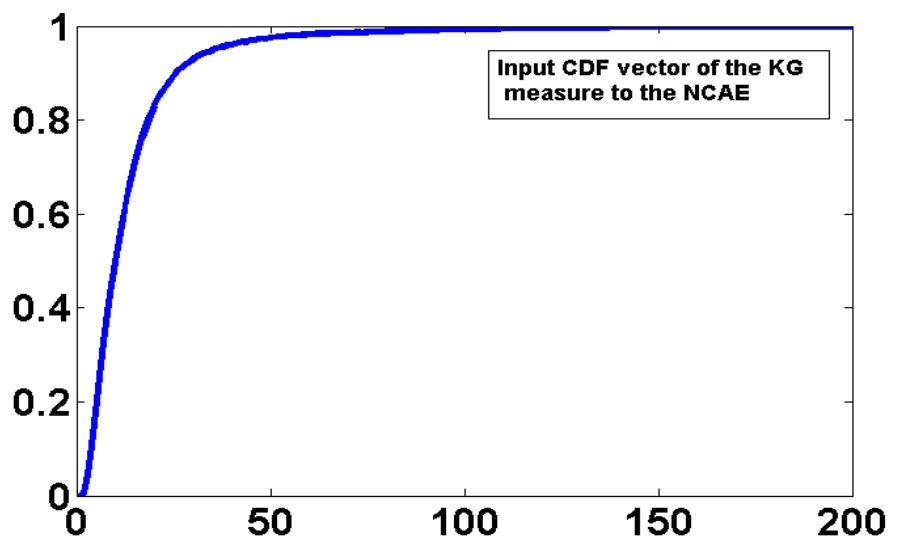

(a)

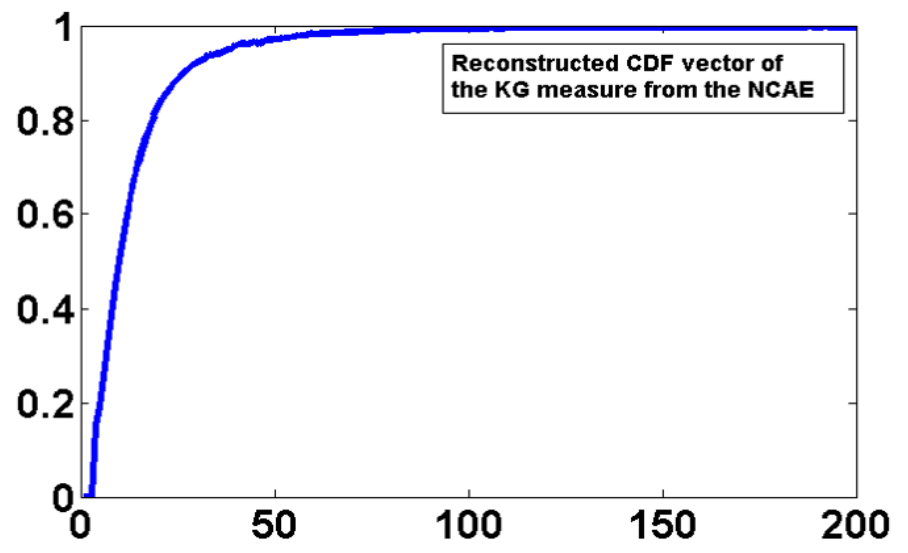

(b)

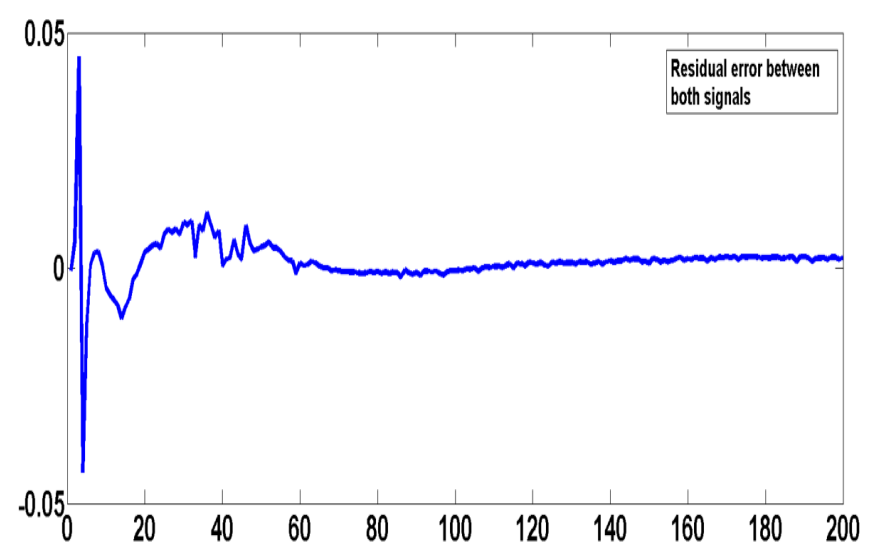

(c)

FIGURE 27: (a) Original input CDF vector of one of the Cx measures, $K_{G}$, to the NCAE. (b) reconstructed CDF vector from the NCAE. (c) reconstruction error between both. 


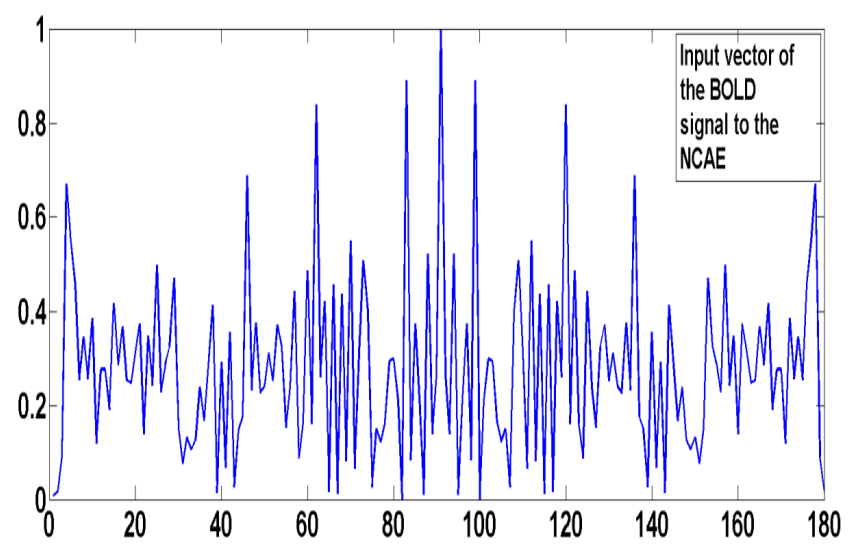

(a)

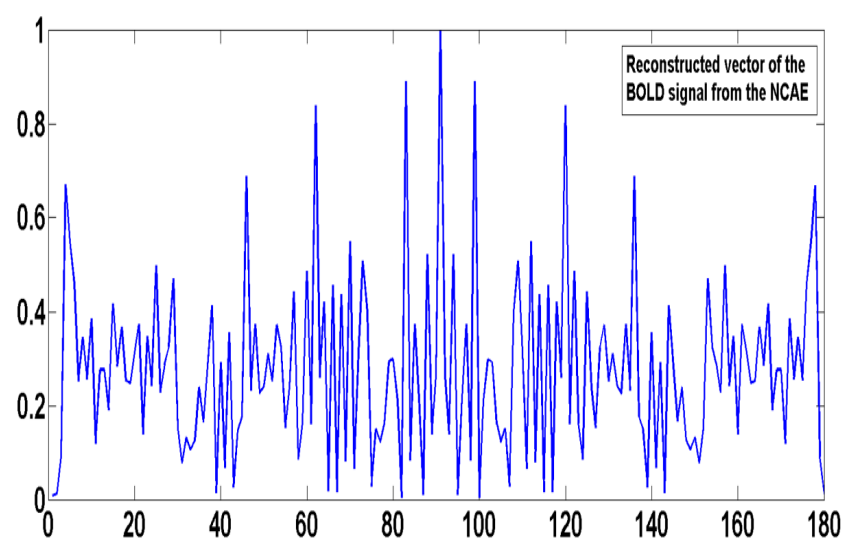

(b)

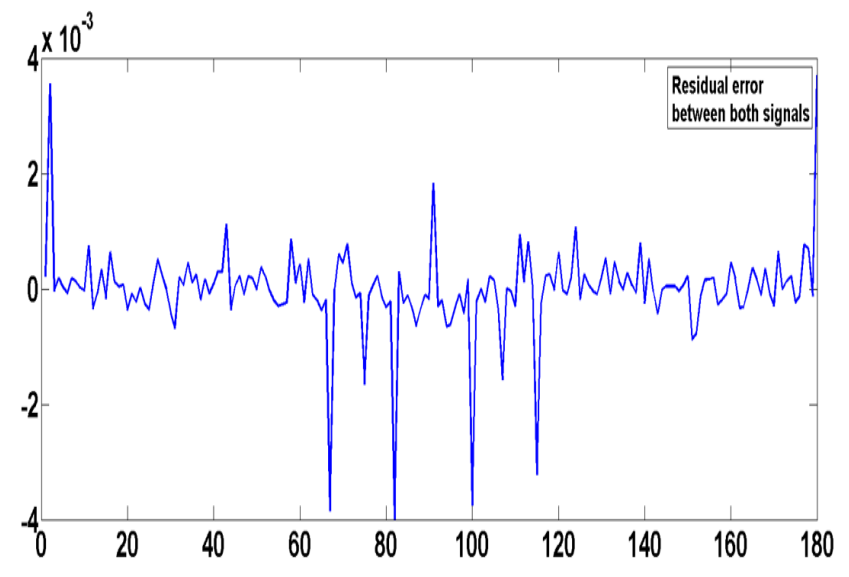

(c)

FIGURE 28: (a) Original input vector of the fMRI signal to the NCAE. (b) reconstructed vector from the NCAE. (c) reconstruction error between both. 


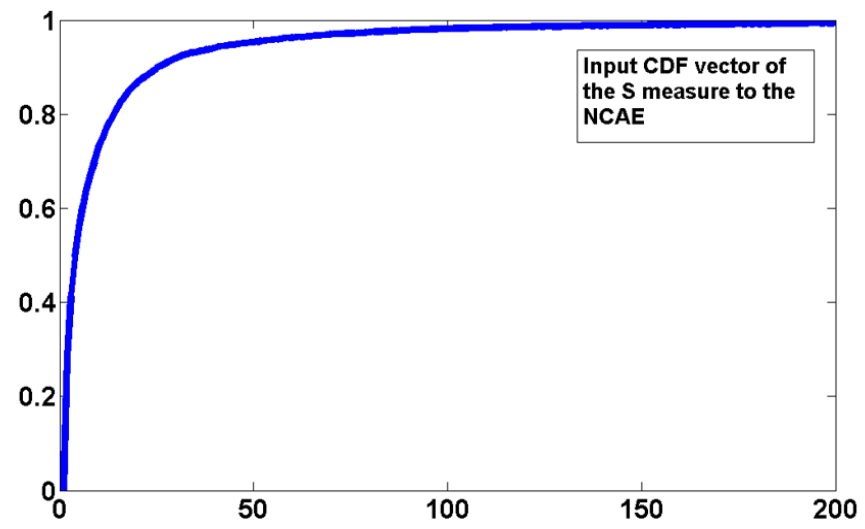

(a)

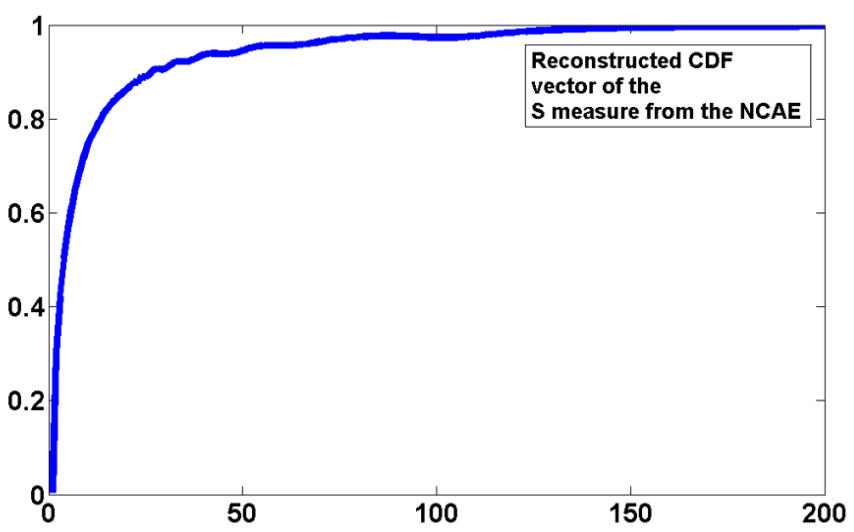

(b)

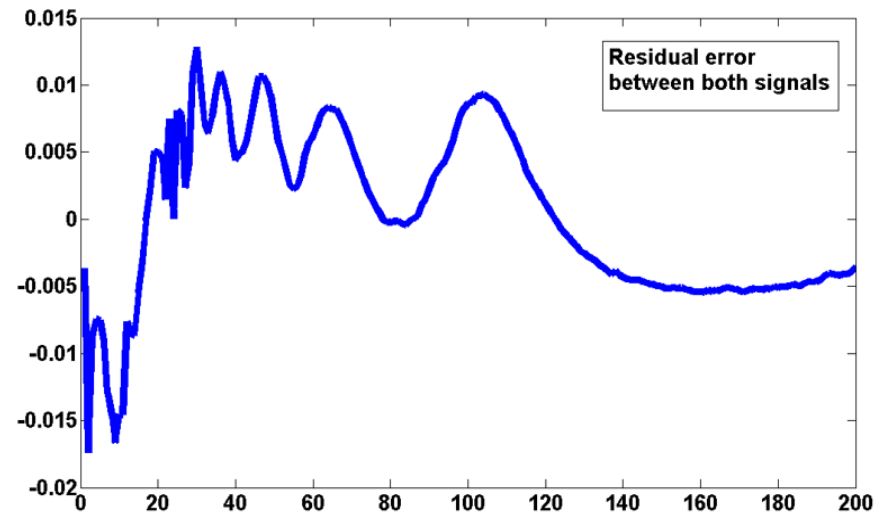

(c)

FIGURE 29: (a) Original input CDF vector of one of the CWM measures, $S$, to the NCAE. (b) reconstructed CDF vector from the NCAE. (c) reconstruction error between both. 


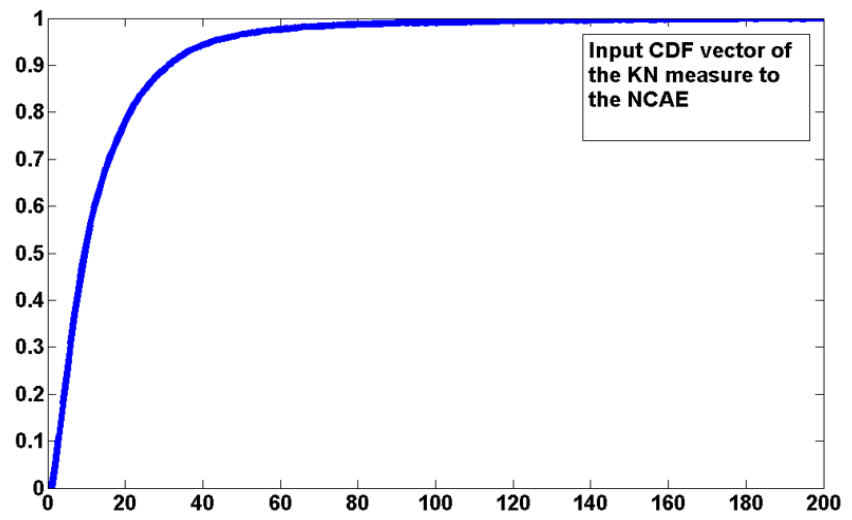

(a)

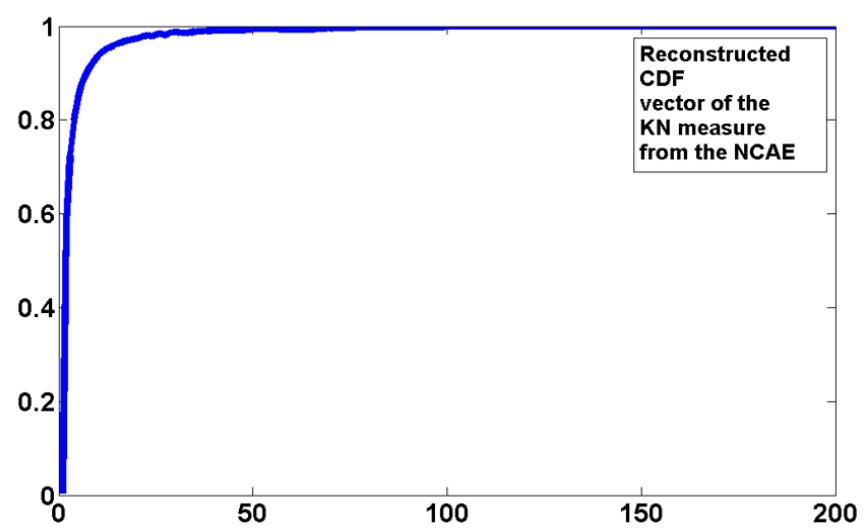

(b)

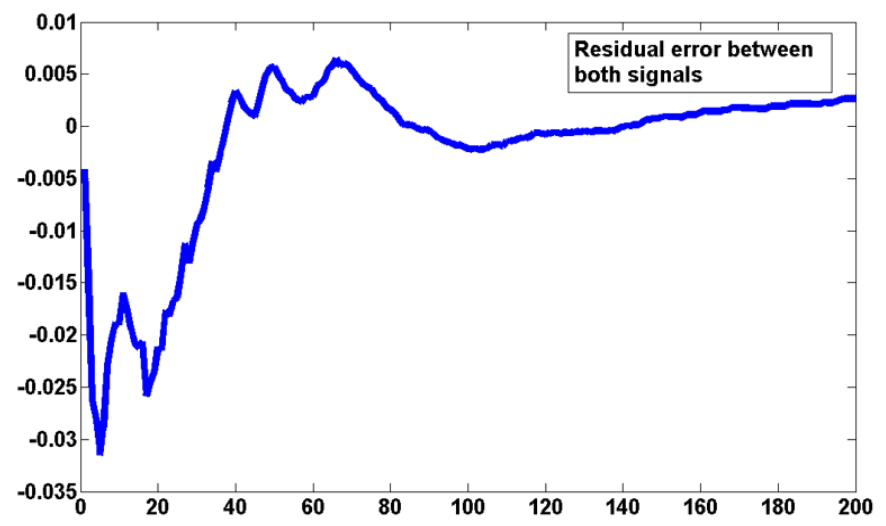

(c)

FIGURE 30: (a) Original input CDF vector of one of the $\mathrm{Cx}$ measures, $K_{N}$, to the NCAE. (b) reconstructed CDF vector from the NCAE. (c) reconstruction error between both. 


\section{CHAPTER IV \\ CONCLUSIONS AND FUTURE WORK}

\section{A. Conclusions}

To conclude, this work presented a novel automated CAD system for autism diagnosis using sMRI and fMRI data. The proposed framework mainly relies on stripping the skull from brain scans, followed by applying an accurate segmentation framework on MR brain images using a joint model that combines shape, intensity, and spatial information. The segmentation algorithm was validated on several databases that span different life stages showing high segmentation accuracy, and also outperformed other state-of-theart approaches.

After obtaining the segmented brain volumes, CWM and Cx meshes are constructed, from which shape features are extracted (sMRI experiment) as well as features related to the functional activity of the brain that corresponds to a monetary reward task (fMRI experiment). For the sMRI experiment, SPAHRM is used to construct the Cx meshes, followed by computing four shape features. Four other shape features are computed from CWM meshes. Also, a monetary reward fMRI experiment is conducted, where brain activation was modeled using the SPM package. In all subjects, voxels that exhibited the most significant BOLD signal activation change due to the reward feedback event are extracted and sorted, to be then used for classification. Finally, all extracted features from both experiments are fed into a deep fusion classification network for classification and diagnosis. The CAD system was applied on a wide database and showed a high global classification accuracy between autistic and typically developing brains of $94.74 \%$. It also provided local 
analysis for areas of the brain based on two labeling atlases, which can resolve autism endophenotypes and help clinicians deliver personalized treatments to individuals with ASD. The integration of imaging modalities such as sMRI and fMRI data is sought in this work in hopes to increase the potential and robustness of the proposed CAD system.

\section{B. Future Work}

Future directions of research for this thesis can be outlined as follows:

1- In addition to the SBM study conducted in this work, The BioImaging group has a huge interest in combining behavior reports with the findings from the present study to better understand and assess ASD.

2- Moreover, this work also aims at investigating the shape features of other deep structures that have shown correlation with autism, such as the corpus callosum and the hippocampus.

This work could also be applied to various other applications in medical imaging, such as the kidney, the heart, the prostate, the lung, and the retina.

One application is renal transplant functional assessment. Chronic kidney disease (CKD) affects about 26 million people in U.S. with 17,000 transplants being performed each year $[352,353]$. In renal transplant patients, acute rejection is the leading cause of renal dysfunction. Given the limited number of donors, routine clinical posttransplantation evaluation is of immense importance to help clinicians initiate timely interventions with appropriate treatment and thus prevent the graft loss. In recent years an increased area of research has been dedicated to developing noninvasive computer-aided diagnostic (CAD) systems for renal transplant function assessment, utilizing different image modalities (e.g., ultrasound (US), computed tomography (CT), magnetic resonance imaging (MRI), etc.). Accurate assessment of renal transplant function is critically important for graft survival [354]. Although transplantation can improve a patient wellbeing, there is a potential post-transplantation risk of kidney dysfunction that, if not treated in a timely 
manner, can lead to the loss of the entire graft, and even patient death. Thus, accurate assessment of renal transplant function is crucial for the identification of proper treatment. In recent years, an increased area of research has been dedicated to developing non-invasive image-based CAD systems for the assessment of renal transplant function. In particular, dynamic [355-362] and diffusion [363-372] MRI-based systems have been clinically used to assess transplanted kidneys with the advantage of providing information on each kidney separately [373-386].

Another important application for this work could be the prostate. Prostate cancer is the second leading cause of cancer-related male deaths among Americans. Recent cancer studies reported an estimate of 220,800 new cases diagnosed and a mortality rate close to 27,540 from prostate cancer in 2015. Also, one in seven men is expected to be diagnosed with the disease in their lifetime. Fortunately, early detection of prostate cancer can enhance the survival rate since prostate cancer is better treated at early stages of the disease. This motivated researchers to develop various non-invasive diagnostic system for early detection of prostate cancer, such as the work in [387-399].

The heart is also an important application to this work. The clinical assessment of myocardial perfusion plays a major role in the diagnosis, management, and prognosis of ischemic heart disease patients. Thus, there have been ongoing efforts to develop automated systems for accurate analysis of myocardial perfusion using first-pass images [400-421].

Another application for this work could be the detection of retinal abnormalities. Majority of ophthalmologists depend on the visual interpretation for the identification of diseases types. However, inaccurate diagnosis will affect the treatment procedure which may lead to fatal results. Hence, there is a crucial need for computer automated diagnosis systems that yield highly accurate results. Optical coherence tomography (OCT) has become a powerful modality for the non-invasive diagnosis of various retinal abnormalities such as glaucoma, diabetic macular edema, and macular degeneration. The problem with diabetic retinopathy (DR) is that the patient is not aware of the disease until the changes in 
the retina have progressed to a level that treatment tends to be less effective. Therefore, automated early detection could limit the severity of the disease and assist ophthalmologists in investigating and treating it more efficiently [422-425].

Abnormalities of the lung could also be another promising area of research and a related application to this work. Radiation-induced lung injury is the main side effect of radiation therapy for lung cancer patients. Although higher radiation doses increase the radiation therapy effectiveness for tumor control, this can lead to lung injury as a greater quantity of normal lung tissues is included in the treated area. Almost $13 \%$ 37\% of patients who undergo radiation therapy develop lung injury following radiation treatment. The severity of Radiation-induced lung injury ranges from ground-glass opacities and consolidation at the early phase to fibrosis and traction bronchiectasis in the late phase. Early detection of lung injury will thus help to improve management of the treatment [426-433].

This work can also be applied to other brain abnormalities, such as Dyslexia. Dyslexia is one of the most complicated developmental brain disorders that affect childrens learning abilities. Dyslexia leads to the failure to develop age-appropriate reading skills in spite of the normal intelligence level and adequate reading instructions. Neuropathological studies have revealed an abnormal anatomy of some structures, such as the Corpus Callosum in dyslexic brains. There has been a lot of work in the literature that aims at developing CAD systems for diagnosing such disorder, along with other brain disorders [327, 331, 434-463].

This work could also be applied for the extraction of blood vessels from phase contrast (PC) magnetic resonance angiography (MRA). Accurate cerebrovascular segmentation using non-invasive MRA is crucial for the early diagnosis and timely treatment of intracranial vascular diseases [464-468]. 


\section{REFERENCES}

[1] NIMH. Autism Spectrum Disorder. Office of Science Policy, Bethesda, 2015.

[2] Christopher Gillberg. Autism and pervasive developmental disorders. Journal of Child Psychology and Psychiatry, 31(1):99-119, 1990.

[3] American Psychiatric Association et al. Diagnostic and statistical manual of mental disorders (DSM-5@). American Psychiatric Pub, 2013.

[4] Catherine Lord, Michael Rutter, Pamela C Dilavore, Susan Risi, Bernadette Rogé, Eric Fombonne, Jeanne Fremolle-Kruck, and Evelyne Arti. ADOS: Autism Diagnostic Observation Schedule, volume 1. Western Psychological Services Los Angeles, CA, 2008.

[5] Catherine Lord, Michael Rutter, and Ann Le Couteur. Autism diagnostic interviewrevised: a revised version of a diagnostic interview for caregivers of individuals with possible pervasive developmental disorders. Journal of autism and developmental disorders, 24(5):659-685, 1994.

[6] David Wechsler. Wechsler, manual for the wechsler intelligence scale for children fourth edition manual. The Psychological Corporation, San Antonio, TX: Psychological Corporation Harcourt Brace Jo vanovich, 1991.

[7] Diana L Robins, Deborah Fein, Marianne L Barton, and James A Green. The modified checklist for autism in toddlers: an initial study investigating the early detection of autism and pervasive developmental disorders. Journal of autism and developmental disorders, 31(2):131-144, 2001.

[8] Joseph Piven, Stephan Arndt, James Bailey, Susan Havercamp, Nancy C Andreasen, and Pat Palmer. An mri study of brain size in autism. American Journal of Psychiatry, 152(8):1145-1149, 1995.

[9] Aea Bailey, P Luthert, A Dean, B Harding, I Janota, M Montgomery, M Rutter, and P Lantos. A clinicopathological study of autism. Brain, 121(5):889-905, 1998.

[10] Manuel F Casanova, Daniel P Buxhoeveden, Andrew E Switala, and Emil Roy. Minicolumnar pathology in autism. Neurology, 58(3):428-432, 2002.

[11] Manueal F Casanova and Andrew E Switala. Minicolumnar morphometry: computerized image analysis. Neocortical modularity and the cell minicolumn. Nova Biomedical, New York, pages 161-180, 2005.

[12] Manuel F Casanova, Daniel P Buxhoeveden, Andrew E Switala, and Emil Roy. Neuronal density and architecture (gray level index) in the brains of autistic patients. Journal of Child Neurology, 17(7):515-521, 2002.

[13] Antonio R Damasio and Ralph G Maurer. A neurological model for childhood autism. Archives of neurology, 35(12):777-786, 1978. 
[14] Margaret Bauman and Thomas L Kemper. Histoanatomic observations of the brain in early infantile autism. Neurology, 35(6):866-866, 1985.

[15] Gary R Gaffney, Luke Y Tsai, Samuel Kuperman, and Susan Minchin. Cerebellar structure in autism. American journal of diseases of children, 141(12):1330-1332, 1987.

[16] Gary R Gaffney, Samuel Kuperman, Luke Y Tsai, Susan Minchin, and Khatab M Hassanein. Midsagittal magnetic resonance imaging of autism. The British Journal of Psychiatry, 151(6):831-833, 1987.

[17] Eric Courchesne, R Yeung-Courchesne, JR Hesselink, and TL Jernigan. Hypoplasia of cerebellar vermal lobules vi and vii in autism. New England Journal of Medicine, 318(21):1349-1354, 1988.

[18] Gary R Gaffney, Samuel Kuperman, Luke Y Tsai, and Susan Minchin. Morphological evidence for brainstem involvement in infantile autism. Biological Psychiatry, 24(5):578-586, 1988.

[19] Barry Horwitz, Judith M Rumsey, Cheryl L Grady, and Stanley I Rapoport. The cerebral metabolic landscape in autism: intercorrelations of regional glucose utilization. Archives of neurology, 45(7):749-755, 1988.

[20] Nancy J Minshew and James B Payton. New perspectives in autism, part i: The clinical spectrum of autism. Current Problems in Pediatrics, 18(10):567-610, 1988.

[21] Edward R Ritvo and HJ Garber. Cerebellar hypoplasia and autism. New England Journal of Medicine, 319(11):1152-1154, 1988.

[22] Gary R Gaffney, Samuel Kuperman, Luke Y Tsai, and Susan Minchin. Forebrain structure in infantile autism. Journal of the American Academy of Child \& Adolescent Psychiatry, 28(4):534-537, 1989.

[23] James W Murakami, Eric Courchesne, Gary A Press, Rachel Yeung-Courchesne, and John R Hesselink. Reduced cerebellar hemisphere size and its relationship to vermal hypoplasia in autism. Archives of Neurology, 46(6):689-694, 1989.

[24] Jordan Garber, Edward R Ritvo, Lee C Chiu, Virginia J Griswold, Alona Kashanian, BJ Freeman, and William H Oldendorf. A magnetic resonance imaging study of autism: Normal fourth ventricle size and absence of pathology. The American journal of psychiatry, 1989.

[25] AL Gropman, B Gertz, K Shattuck, IL Kahn, R Seltzer, L Krivitsky, and J Van Meter. Diffusion tensor imaging detects areas of abnormal white matter microstructure in patients with partial ornithine transcarbamylase deficiency. American Journal of Neuroradiology, 31(9):1719-1723, 2010.

[26] Susumu Mori and J-Donald Tournier. Introduction to Diffusion Tensor Imaging and Higher Order Models. Academic Press, 2013.

[27] Ruth CM Philip, Maria R Dauvermann, Heather C Whalley, Katie Baynham, Stephen M Lawrie, and Andrew C Stanfield. A systematic review and meta-analysis of the fmri investigation of autism spectrum disorders. Neuroscience \& Biobehavioral Reviews, 36(2):901-942, 2012. 
[28] Ralph-Axel Müller, Patricia Shih, Brandon Keehn, Janae R Deyoe, Kelly M Leyden, and Dinesh K Shukla. Underconnected, but how? a survey of functional connectivity mri studies in autism spectrum disorders. Cerebral Cortex, 21(10):2233-2243, 2011.

[29] Peter J Basser, James Mattiello, and Denis LeBihan. Estimation of the effective selfdiffusion tensor from the NMR spin echo. Journal of Magnetic Resonance, Series B, 103(3):247-254, 1994.

[30] Peter J Basser, James Mattiello, and Denis LeBihan. Mr diffusion tensor spectroscopy and imaging. Biophysical journal, 66(1):259-267, 1994.

[31] Qian Dong, Robert C Welsh, Thomas L Chenevert, Ruth C Carlos, Pia MalySundgren, Diana M Gomez-Hassan, and Suresh K Mukherji. Clinical applications of diffusion tensor imaging. Journal of Magnetic Resonance Imaging, 19(1):6-18, 2004.

[32] Peter J Basser, Sinisa Pajevic, Carlo Pierpaoli, Jeffrey Duda, and Akram Aldroubi. In vivo fiber tractography using dt-mri data. Magnetic resonance in medicine, 44(4):625-632, 2000.

[33] Pallavi Rane, David Cochran, Steven M Hodge, Christian Haselgrove, David N Kennedy, and Jean A Frazier. Connectivity in autism: A review of mri connectivity studies. Harvard review of psychiatry, 23(4):223-244, 2015.

[34] Karen Blackmon. Structural mri biomarkers of shared pathogenesis in autism spectrum disorder and epilepsy. Epilepsy \& Behavior, 47:172-182, 2015.

[35] Eugenia Conti, Sara Calderoni, Viviana Marchi, Filippo Muratori, Giovanni Cioni, and Andrea Guzzetta. The first 1000 days of the autistic brain: a systematic review of diffusion imaging studies. Frontiers in human neuroscience, 9, 2015.

[36] Saskia JMC Palmen and Herman van Engeland. Review on structural neuroimaging findings in autism. Journal of Neural Transmission, 111(7):903-929, 2004.

[37] Adel M Zeglam, Marwa F Al-Ogab, and Thouraya Al-Shaftery. Mri or not to mri! should brain mri be a routine investigation in children with autistic spectrum disorders? Acta Neurologica Belgica, 115(3):351-354, 2015.

[38] Mark S George, Durval C Costa, Kypros Kouris, Howard A Ring, and Peter J Ell. Cerebral blood flow abnormalities in adults with infantile autism. The Journal of nervous and mental disease, 180(7):413-417, 1992.

[39] Jordan Garber and Edward R Ritvo. Magnetic resonance imaging of the posterior fossa in autistic adults. The American journal of psychiatry, 1992.

[40] John R Holttum, Nancy J Minshew, Richard S Sanders, and Nancie E Phillips. Magnetic resonance imaging of the posterior fossa in autism. Biological Psychiatry, 32(12):1091-1101, 1992.

[41] Joseph Piven, Eileen Nehme, Jon Simon, Patrick Barta, Godfrey Pearlson, and Susan E Folstein. Magnetic resonance imaging in autism: measurement of the cerebellum, pons, and fourth ventricle. Biological Psychiatry, 31(5):491-504, 1992.

[42] Michele D Kleiman, Samuel Neff, and N Paul Rosman. The brain in infantile autism are posterior fossa structures abnormal? Neurology, 42(4):753-753, 1992. 
[43] T Hashimoto, K Murakawa, M Miyazaki, M Tayama, and Y Kuroda. Magnetic resonance imaging of the brain structures in the posterior fossa in retarded autistic children. Acta Paediatrica, 81(12):1030-1034, 1992.

[44] Toshiaki Hashimoto, Masanobu Tayama, Masahito Miyazaki, Kazuyoshi Murakawa, and Yasuhiro Kuroda. Brainstem and cerebellar vermis involvement in autistic children. Journal of child neurology, 8(2):149-153, 1993.

[45] E Courchesne, GA Press, and R Yeung-Courchesne. Parietal lobe abnormalities detected with $\mathrm{mr}$ in patients with infantile autism. AJR. American journal of roentgenology, 160(2):387-393, 1993.

[46] Anthony Bailey, Phillip Luthert, Patrick Bolton, Anne Le Couteur, Michael Rutter, and Brian Harding. Autism and megalencephaly. The Lancet, 341(8854):12251226, 1993.

[47] Ralph Adolphs, Daniel Tranel, Hanna Damasio, and Antonio Damasio. Impaired recognition of emotion in facial expressions following bilateral damage to the human amygdala. Nature, 372(6507):669-672, 1994.

[48] Martha Ann Nowell, David B Hackney, Alan S Muraki, and Mary Coleman. Varied $\mathrm{mr}$ appearance of autism: fifty-three pediatric patients having the full autistic syndrome. Magnetic resonance imaging, 8(6):811-816, 1990.

[49] Melissa Hsu, Rachel Yeung-Courchesne, Eric Courchesne, and Gary A Press. Absence of magnetic resonance imaging evidence of pontine abnormality in infantile autism. Archives of neurology, 48(11):1160-1163, 1991.

[50] Stuart Zola-Morgan, Larry R Squire, Robert P Clower, and Pablo Alvarez-Royo. Independence of memory functions and emotional behavior: separate contributions of the hippocampal formation and the amygdala. Hippocampus, 1(2):207-220, 1991.

[51] Margaret L Bauman and Thomas L Kemper. Neuroanatomic observations of the brain in autism. The neurobiology of autism, 612:119-145, 1994.

[52] Jocelyne Bachevalier. Medial temporal lobe structures and autism: a review of clinical and experimental findings. Neuropsychologia, 32(6):627-648, 1994.

[53] NJ Minshew and SM Dombrowski. In vivo neuroanatomy of autism: neuroimaging studies. The neurobiology of autism, pages 66-85, 1994.

[54] Eric Courchesne, Jeanne Townsend, and Osamu Saitoh. The brain in infantile autism posterior fossa structures are abnormal. Neurology, 44(2):214-214, 1994.

[55] E Courchesne, O Saitoh, R Yeung-Courchesne, GA Press, AJ Lincoln, RH Haas, and L Schreibman. Abnormality of cerebellar vermian lobules vi and vii in patients with infantile autism: identification of hypoplastic and hyperplastic subgroups with mr imaging. AJR. American journal of roentgenology, 162(1):123-130, 1994.

[56] O Saitoh, Eric Courchesne, B Egaas, AJ Lincoln, and L Schreibman. Cross-sectional area of the posterior hippocampus in autistic patients with cerebellar and corpus callosum abnormalitigs. Neurology, 45(2):317-324, 1995.

[57] Brian Egaas, Eric Courchesne, and Osamu Saitoh. Reduced size of corpus callosum in autism. Archives of Neurology, 52(8):794-801, 1995. 
[58] Toshiaki Hashimoto, Masanobu Tayama, Kazuyosi Murakawa, Tsutomu Yoshimoto, Masahito Miyazaki, Midori Harada, and Yasuhiro Kuroda. Development of the brainstem and cerebellum in autistic patients. Journal of autism and developmental disorders, 25(1):1-18, 1995.

[59] Monica Zilbovicius, Bernard Garreau, Yves Samson, Philippe Remy, Catherine Barthelemy, Andre Syrota, and Gilbert Lelord. Delayed maturation of the frontal cortex in childhood autism. American Journal of Psychiatry, 152(2):248-252, 1995.

[60] G Bradley Schaefer, James N Thompson, John B Bodensteiner, James M McConnell, William J Kimberling, Charles T Gay, William D Dutton, David C Hutchings, and Stanton B Gray. Hypoplasia of the cerebellar vermis in neurogenetic syndromes. Annals of neurology, 39(3):382-385, 1996.

[61] Don J Siegel, Nancy J Minshew, and Gerald Goldstein. Wechsler iq profiles in diagnosis of high-functioning autism. Journal of autism and developmental disorders, 26(4):389-406, 1996.

[62] Jay N Giedd, John W Snell, Nicholas Lange, Jagath C Rajapakse, BJ Casey, Patricia L Kozuch, A Catherine Vaituzis, Yolanda C Vauss, Susan D Hamburger, Debra Kaysen, and Judith L Rapoport. Quantitative magnetic resonance imaging of human brain development: ages 4-18. Cerebral cortex, 6(4):551-559, 1996.

[63] Joseph Piven, Stephan Arndt, James Bailey, and Nancy Andreasen. Regional brain enlargement in autism: a magnetic resonance imaging study. Journal of the American Academy of Child \& Adolescent Psychiatry, 35(4):530-536, 1996.

[64] M Elia, L Manfre, R Ferri, SA Musumeci, S Panerai, M Bottitta, C Scuderi, S DelGracco, and MC Stefanini. Brain morphometry and psychobehavioural measures in autistic low-functioning subjects. RIVISTA DI NEURORADIOLOGIA, 10(4):431436, 1997.

[65] Joseph Piven, James Bailey, Bonnie J Ranson, and Stephan Arndt. An mri study of the corpus callosum in autism. American Journal of Psychiatry, 154(8):1051-1056, 1997.

[66] Kristina T Ciesielski, Richard J Harris, Blaine L Hart, and Henry F Pabst. Cerebellar hypoplasia and frontal lobe cognitive deficits in disorders of early childhood. Neuropsychologia, 35(5):643-655, 1997.

[67] Janet E Lainhart, Joseph Piven, Maryann Wzorek, Rebecca Landa, Susan L Santangelo, Hilary Coon, and Susan E Folstein. Macrocephaly in children and adults with autism. Journal of the American Academy of Child \& Adolescent Psychiatry, 36(2):282-290, 1997.

[68] Joseph Piven, James Bailey, Bonnie J Ranson, and Stephan Arndt. No difference in hippocampus volume detected on magnetic resonance imaging in autistic individuals. Journal of Autism and Developmental Disorders, 28(2):105-110, 1998.

[69] Geraldine Dawson, Andrew N Meltzoff, Julie Osterling, and Julie Rinaldi. Neuropsychological correlates of early symptoms of autism. Child development, 69(5):1276-1285, 1998. 
[70] Christopher Gillberg. Neurodevelopmental processes and psychological functioning in autism. Development and Psychopathology, 11(03):567-587, 1999.

[71] Facundo Manes, Joseph Piven, Daniela Vrancic, Valeria Nanclares, Christian Plebst, and Sergio E Starkstein. An mri study of the corpus callosum and cerebellum in mentally retarded autistic individuals. The Journal of neuropsychiatry and clinical neurosciences, 11(4):470-474, 1999.

[72] Frances Abell, Michael Krams, John Ashburner, Richard Passingham, Karl Friston, Richard Frackowiak, Francesca Happé, Chris Frith, and Uta Frith. The neuroanatomy of autism: a voxel-based whole brain analysis of structural scans. $\mathrm{Neu}$ roreport, 10(8):1647-1651, 1999.

[73] Jennifer G Levitt, Rebecca Blanton, Linda Capetillo-Cunliffe, Donald Guthrie, Arthur Toga, and James T McCracken. Cerebellar vermis lobules viiix in autism. Progress in Neuro-Psychopharmacology and Biological Psychiatry, 23(4):625-633, 1999.

[74] Lonnie L Sears, Cortney Vest, Somaia Mohamed, James Bailey, Bonnie J Ranson, and Joseph Piven. An mri study of the basal ganglia in autism. Progress in NeuroPsychopharmacology and Biological Psychiatry, 23(4):613-624, 1999.

[75] Jeanne Townsend, Eric Courchesne, James Covington, Marissa Westerfield, Naomi Singer Harris, Patrick Lyden, Timothy P Lowry, and Gary A Press. Spatial attention deficits in patients with acquired or developmental cerebellar abnormality. The Journal of Neuroscience, 19(13):5632-5643, 1999.

[76] EH Aylward, NJ Minshew, G Goldstein, NA Honeycutt, AM Augustine, KO Yates, PE Barta, and GD Pearlson. Mri volumes of amygdala and hippocampus in nonmentally retarded autistic adolescents and adults. Neurology, 53(9):2145-2145, 1999.

[77] Eric Courchesne, Ralph-Axel Müller, and Osamu Saitoh. Brain weight in autism: normal in the majority of cases, megalencephalic in rare cases. Neurology, 52(5):1057-1057, 1999.

[78] Eric Fombonne, Bernadette Rogé, Jacques Claverie, Stephanie Courty, and Jeanne Fremolle. Microcephaly and macrocephaly in autism. Journal of autism and developmental disorders, 29(2):113-119, 1999.

[79] Rong Chen, Yun Jiao, and Edward H Herskovits. Structural mri in autism spectrum disorder. Pediatric research, 69:63R-68R, 2011.

[80] Andrew C Stanfield, Andrew M McIntosh, Michael D Spencer, Ruth Philip, Sonia Gaur, and Stephen M Lawrie. Towards a neuroanatomy of autism: a systematic review and meta-analysis of structural magnetic resonance imaging studies. European Psychiatry, 23(4):289-299, 2008.

[81] Paolo Brambilla, Antonio Hardan, Stefania Ucelli di Nemi, Jorge Perez, Jair C Soares, and Francesco Barale. Brain anatomy and development in autism: review of structural mri studies. Brain research bulletin, 61(6):557-569, 2003.

[82] Jan J. Koenderink and Andrea J. Van Doorn. Surface shape and curvature scales. Image and vision computing, 10(8):557-564, 1992. 
[83] Rudolph Pienaar, B. Fischl, V. Caviness, N. Makris, and P.E. Grant. A methodology for analyzing curvature in the developing brain from preterm to adult. IJIST, 18(1):42-68, 2008.

[84] Rita Carter. The human brain book. Penguin, 2014.

[85] Heather Cody Hazlett, Michele Poe, Guido Gerig, Rachel Gimpel Smith, James Provenzale, Allison Ross, John Gilmore, and Joseph Piven. Magnetic resonance imaging and head circumference study of brain size in autism: birth through age 2 years. Archives of general psychiatry, 62(12):1366-1376, 2005.

[86] Cynthia M Schumann, Cinnamon S Bloss, Cynthia Carter Barnes, Graham M Wideman, Ruth A Carper, Natacha Akshoomoff, Karen Pierce, Donald Hagler, Nicholas Schork, Catherine Lord, and Eric Courchesne. Longitudinal magnetic resonance imaging study of cortical development through early childhood in autism. The Journal of Neuroscience, 30(12):4419-4427, 2010.

[87] Heather Cody Hazlett, Hongbin Gu, Robert C McKinstry, Dennis WW Shaw, Kelly N Botteron, Stephen R Dager, Martin Styner, Clement Vachet, Guido Gerig, Sarah J Paterson, Robert T Schultz, Annette M Estes, Alan C Evans, and Joseph Piven. Brain volume findings in 6-month-old infants at high familial risk for autism. American Journal of Psychiatry, 169(6):601-608, 2012.

[88] Mark D Shen, Christine W Nordahl, Gregory S Young, Sandra L Wootton-Gorges, Aaron Lee, Sarah E Liston, Kayla R Harrington, Sally Ozonoff, and David G Amaral. Early brain enlargement and elevated extra-axial fluid in infants who develop autism spectrum disorder. Brain, page awt166, 2013.

[89] Eric Courchesne, CM Karns, HR Davis, R Ziccardi, RA Carper, ZD Tigue, HJ Chisum, P Moses, K Pierce, C Lord, AJ Lincoln, S Pizzo, L Schreibman, RH Haas, NA Akshoomoff, and RY Courchesne. Unusual brain growth patterns in early life in patients with autistic disorder an mri study. Neurology, 57(2):245-254, 2001.

[90] Ruth A Carper, Pamela Moses, Zachary D Tigue, and Eric Courchesne. Cerebral lobes in autism: early hyperplasia and abnormal age effects. Neuroimage, 16(4):1038-1051, 2002.

[91] BF Sparks, SD Friedman, DW Shaw, EH Aylward, D Echelard, AA Artru, KR Maravilla, JN Giedd, J Munson, G Dawson, and SR Dager. Brain structural abnormalities in young children with autism spectrum disorder. Neurology, 59(2):184-192, 2002.

[92] Natacha Akshoomoff, Catherine Lord, Alan J Lincoln, Rachel Y Courchesne, Ruth A Carper, Jeanne Townsend, and Eric Courchesne. Outcome classification of preschool children with autism spectrum disorders using mri brain measures. Journal of the American Academy of Child \& Adolescent Psychiatry, 43(3):349-357, 2004.

[93] Nathalie Boddaert, Nadia Chabane, H Gervais, CD Good, M Bourgeois, MH Plumet, C Barthelemy, MC Mouren, E Artiges, Y Samson, F Brunelle, RSJ Frackowiak, and M Zilbovicius. Superior temporal sulcus anatomical abnormalities in childhood autism: a voxel-based morphometry mri study. Neuroimage, 23(1):364-369, 2004. 
[94] Gráinne M McAlonan, Vinci Cheung, Charlton Cheung, John Suckling, Grace Y Lam, KS Tai, L Yip, Declan GM Murphy, and Siew E Chua. Mapping the brain in autism. a voxel-based mri study of volumetric differences and intercorrelations in autism. Brain, 128(2):268-276, 2005.

[95] Ragy R Girgis, Nancy J Minshew, Nadine M Melhem, Jeffrey J Nutche, Matcheri S Keshavan, and Antonio Y Hardan. Volumetric alterations of the orbitofrontal cortex in autism. Progress in Neuro-Psychopharmacology and Biological Psychiatry, 31(1):41-45, 2007.

[96] Caroline C Brun, Rob Nicolson, Natasha Leporé, Yi-Yu Chou, Christine N Vidal, Timothy J DeVito, Dick J Drost, Peter C Williamson, Nagalingam Rajakumar, Arthur W Toga, and Paul M Thompson. Mapping brain abnormalities in boys with autism. Human brain mapping, 30(12):3887-3900, 2009.

[97] Antonio Y Hardan, Sri Muddasani, Madhuri Vemulapalli, Matcheri S Keshavan, and Nancy J Minshew. An mri study of increased cortical thickness in autism. Americal Journal of Psychiatry, 163(7):1290-1292, 2006.

[98] Antonio Y Hardan, Robin A Libove, Matcheri S Keshavan, Nadine M Melhem, and Nancy J Minshew. A preliminary longitudinal magnetic resonance imaging study of brain volume and cortical thickness in autism. Biological psychiatry, 66(4):320-326, 2009.

[99] Yun Jiao, Rong Chen, Xiaoyan Ke, Kangkang Chu, Zuhong Lu, and Edward H Herskovits. Predictive models of autism spectrum disorder based on brain regional cortical thickness. Neuroimage, 50(2):589-599, 2010.

[100] Donna L Dierker, Eric Feczko, John R Pruett, Steven E Petersen, Bradley L Schlaggar, John N Constantino, John W Harwell, Timothy S Coalson, and David C Van Essen. Analysis of cortical shape in children with simplex autism. Cerebral Cortex, page bht294, 2013.

[101] Ilaria Gori, Alessia Giuliano, Filippo Muratori, Irene Saviozzi, Piernicola Oliva, Raffaella Tancredi, Angela Cosenza, Michela Tosetti, Sara Calderoni, and Alessandra Retico. Gray matter alterations in young children with autism spectrum disorders: comparing morphometry at the voxel and regional level. Journal of Neuroimaging, 25(6):866-874, 2015.

[102] Gordon D Waiter, Justin HG Williams, Alison D Murray, Anne Gilchrist, David I Perrett, and Andrew Whiten. A voxel-based investigation of brain structure in male adolescents with autistic spectrum disorder. Neuroimage, 22(2):619-625, 2004.

[103] Gordon D Waiter, Justin HG Williams, Alison D Murray, Anne Gilchrist, and David I Perrett. Structural white matter deficits in high-functioning individuals with autistic spectrum disorder: a voxel-based investigation. Neuroimage, 24(2):455461, 2005.

[104] Hower Kwon, Andrew W Ow, Kate E Pedatella, Linda J Lotspeich, and Allan L Reiss. Voxel-based morphometry elucidates structural neuroanatomy of highfunctioning autism and asperger syndrome. Developmental Medicine \& Child Neurology, 46(11):760-764, 2004. 
[105] Linda J Lotspeich, Hower Kwon, Cynthia M Schumann, Susanna L Fryer, Beth L Goodlin-Jones, Michael H Buonocore, Cathy R Lammers, David G Amaral, and Allan L Reiss. Investigation of neuroanatomical differences between autism and aspergersyndrome. Archives of general psychiatry, 61(3):291-298, 2004.

[106] Moo K Chung, Kim M Dalton, Andrew L Alexander, and Richard J Davidson. Less white matter concentration in autism: 2d voxel-based morphometry. Neuroimage, 23(1):242-251, 2004.

[107] Heather Cody Hazlett, Michele D Poe, Guido Gerig, Rachel Gimpel Smith, and Joseph Piven. Cortical gray and white brain tissue volume in adolescents and adults with autism. Biological psychiatry, 59(1):1-6, 2006.

[108] Leonardo Bonilha, Fernando Cendes, Chris Rorden, Mark Eckert, Paulo Dalgalarrondo, Li Min Li, and Carlos E Steiner. Gray and white matter imbalancetypical structural abnormality underlying classic autism? Brain and Development, 30(6):396-401, 2008.

[109] Xue Hua, Paul M Thompson, Alex D Leow, Sarah K Madsen, Rochelle Caplan, Jeffry R Alger, Joseph O’Neill, Kishori Joshi, Susan L Smalley, Arthur W Toga, and Jennifer G Levitt. Brain growth rate abnormalities visualized in adolescents with autism. Human brain mapping, 34(2):425-436, 2013.

[110] Antonio Y Hardan, Roger J Jou, Matcheri S Keshavan, Ravi Varma, and Nancy J Minshew. Increased frontal cortical folding in autism: a preliminary mri study. Psychiatry Research: Neuroimaging, 131(3):263-268, 2004.

[111] Christine Wu Nordahl, Donna Dierker, Iman Mostafavi, Cynthia M Schumann, Susan M Rivera, David G Amaral, and David C Van Essen. Cortical folding abnormalities in autism revealed by surface-based morphometry. The Journal of Neuroscience, 27(43):11725-11735, 2007.

[112] Gregory L Wallace, Ian W Eisenberg, Briana Robustelli, Nathan Dankner, Lauren Kenworthy, Jay N Giedd, and Alex Martin. Longitudinal cortical development during adolescence and young adulthood in autism spectrum disorders: Increased cortical thinning but comparable surface area changes. Journal of the American Academy of Child \& Adolescent Psychiatry, 2015.

[113] Antonio Y Hardan, Nancy J Minshew, Mahesh Mallikarjuhn, and Matcheri S Keshavan. Brain volume in autism. Journal of child neurology, 16(6):421-424, 2001.

[114] Donald C Rojas, Eric Peterson, Erin Winterrowd, Martin L Reite, Sally J Rogers, and Jason R Tregellas. Regional gray matter volumetric changes in autism associated with social and repetitive behavior symptoms. BMC psychiatry, 6(1):56, 2006.

[115] F Toal, EM Daly, L Page, Q Deeley, B Hallahan, O Bloemen, WJ Cutter, MJ Brammer, S Curran, D Robertson, C Murphy, KC Murphy, and DGM Murphy. Clinical and anatomical heterogeneity in autistic spectrum disorder: a structural mri study. Psychological medicine, 40(07):1171-1181, 2010.

[116] Nouchine Hadjikhani, Robert M Joseph, Josh Snyder, and Helen Tager-Flusberg. Anatomical differences in the mirror neuron system and social cognition network in autism. Cerebral cortex, 16(9):1276-1282, 2006. 
[117] Krista L Hyde, Fabienne Samson, Alan C Evans, and Laurent Mottron. Neuroanatomical differences in brain areas implicated in perceptual and other core features of autism revealed by cortical thickness analysis and voxel-based morphometry. Human brain mapping, 31(4):556-566, 2010.

[118] Maurizio Elia, Raffaele Ferri, Sebastiano A Musumeci, Simonetta Panerai, Maria Bottitta, and Carmela Scuderi. Clinical correlates of brain morphometric features of subjects with low-functioning autistic disorder. Journal of Child Neurology, 15(8):504-508, 2000.

[119] Walter E Kaufmann, Karen L Cooper, Stewart H Mostofsky, George T Capone, Wendy R Kates, Craig J Newschaffer, Irena Bukelis, Mariah H Stump, Adelene E Jann, and Diane C Lanham. Specificity of cerebellar vermian abnormalities in autism: a quantitative magnetic resonance imaging study. Journal of child neurology, 18(7):463-470, 2003.

[120] Ruth A Carper and Eric Courchesne. Inverse correlation between frontal lobe and cerebellum sizes in children with autism. Brain, 123(4):836-844, 2000.

[121] Antonio Y Hardan, Nancy J Minshew, Keith Harenski, and Matcheri S Keshavan. Posterior fossa magnetic resonance imaging in autism. Journal of the American Academy of Child \& Adolescent Psychiatry, 40(6):666-672, 2001.

[122] MR Herbert, DA Ziegler, CK Deutsch, LM Obrien, N Lange, A Bakardjiev, J Hodgson, KT Adrien, S Steele, N Makris, D Kennedy, GJ Harris, and VS Caviness. Dissociations of cerebral cortex, subcortical and cerebral white matter volumes in autistic boys. Brain, 126(5):1182-1192, 2003.

[123] Roger J Jou, Thomas W Frazier, Matcheri S Keshavan, Nancy J Minshew, and Antonio Y Hardan. A two-year longitudinal pilot mri study of the brainstem in autism. Behavioural brain research, 251:163-167, 2013.

[124] Grainne M McAlonan, Eileen Daly, Veena Kumari, Hugo D Critchley, Therese van Amelsvoort, John Suckling, Andrew Simmons, Thordur Sigmundsson, Kathyrn Greenwood, Ailsa Russell, Nicole Schmitz, Francesca Happe, Patricia Howlin, and DGM Murphy. Brain anatomy and sensorimotor gating in aspergers syndrome. Brain, 125(7):1594-1606, 2002.

[125] Roger J Jou, Nancy J Minshew, Nadine M Melhem, Matcheri S Keshavan, and Antonio Y Hardan. Brainstem volumetric alterations in children with autism. Psychological medicine, 39(08):1347-1354, 2009.

[126] Jeffrey Munson, Geraldine Dawson, Robert Abbott, Susan Faja, Sara Jane Webb, Seth D Friedman, Dennis Shaw, Alan Artru, and Stephen R Dager. Amygdalar volume and behavioral development in autism. Archives of general psychiatry, 63(6):686-693, 2006.

[127] Christine Wu Nordahl, Robert Scholz, Xiaowei Yang, Michael H Buonocore, Tony Simon, Sally Rogers, and David G Amaral. Increased rate of amygdala growth in children aged 2 to 4 years with autism spectrum disorders: a longitudinal study. Archives of general psychiatry, 69(1):53-61, 2012. 
[128] Cynthia Mills Schumann, Julia Hamstra, Beth L Goodlin-Jones, Linda J Lotspeich, Hower Kwon, Michael H Buonocore, Cathy R Lammers, Allan L Reiss, and David G Amaral. The amygdala is enlarged in children but not adolescents with autism; the hippocampus is enlarged at all ages. The Journal of Neuroscience, 24(28):63926401, 2004.

[129] Naama Barnea-Goraly, Thomas W Frazier, Lucia Piacenza, Nancy J Minshew, Matcheri S Keshavan, Allan L Reiss, and Antonio Y Hardan. A preliminary longitudinal volumetric mri study of amygdala and hippocampal volumes in autism. Progress in Neuro-Psychopharmacology and Biological Psychiatry, 48:124-128, 2014.

[130] Donald C Rojas, J Allegra Smith, Tara L Benkers, Suzanne L Camou, Martin L Reite, and Sally J Rogers. Hippocampus and amygdala volumes in parents of children with autistic disorder. American Journal of Psychiatry, 161(11):2038-2044, 2004.

[131] Osamu Saitoh, Christina M Karns, and Eric Courchesne. Development of the hippocampal formation from 2 to 42 years. Brain, 124(7):1317-1324, 2001.

[132] Thomas W Frazier, Matcheri S Keshavan, Nancy J Minshew, and Antonio Y Hardan. A two-year longitudinal mri study of the corpus callosum in autism. Journal of autism and developmental disorders, 42(11):2312-2322, 2012.

[133] Ahmed Elnakib, Manuel F Casanova, G Gimel'farb, Andrew E Switala, and Ayman El-Baz. Autism diagnostics by centerline-based shape analysis of the corpus callosum. In Biomedical Imaging: From Nano to Macro, 2011 IEEE International Symposium on, pages 1843-1846. IEEE, 2011.

[134] Katherine D Tsatsanis, Byron P Rourke, Ami Klin, Fred R Volkmar, Domenic Cicchetti, and Robert T Schultz. Reduced thalamic volume in high-functioning individuals with autism. Biological psychiatry, 53(2):121-129, 2003.

[135] Antonio Y Hardan, Megan Kilpatrick, Matcheri S Keshavan, and Nancy J Minshew. Motor performance and anatomic magnetic resonance imaging (mri) of the basal ganglia in autism. Journal of Child Neurology, 18(5):317-324, 2003.

[136] Antonio Y Hardan, Ragy R Girgis, Jason Adams, Andrew R Gilbert, Matcheri S Keshavan, and Nancy J Minshew. Abnormal brain size effect on the thalamus in autism. Psychiatry Research: Neuroimaging, 147(2):145-151, 2006.

[137] M Mehmet Haznedar, Monte S Buchsbaum, Erin A Hazlett, Elizabeth M LiCalzi, Charles Cartwright, and Eric Hollander. Volumetric analysis and three-dimensional glucose metabolic mapping of the striatum and thalamus in patients with autism spectrum disorders. American Journal of Psychiatry, 163(7):1252-1263, 2006.

[138] Elizabeth H Aylward, NJ Minshew, K Field, BF Sparks, and N Singh. Effects of age on brain volume and head circumference in autism. Neurology, 59(2):175-183, 2002.

[139] Andrew L Alexander, Jee Eun Lee, Mariana Lazar, Rebecca Boudos, Molly B DuBray, Terrence R Oakes, Judith N Miller, Jeffrey Lu, Eun-Kee Jeong, William M McMahon, Erin D Bigler, and Janet E Lainhart. Diffusion tensor imaging of the corpus callosum in autism. Neuroimage, 34(1):61-73, 2007. 
[140] Peter van Gelderen, Marloes HM de Vleeschouwer, Daryl DesPres, James Pekar, Peter van Zijl, and Chrit TW Moonen. Water diffusion and acute stroke. Magnetic Resonance in Medicine, 31(2):154-163, 1994.

[141] Katherine L Narr, Nathan Hageman, Roger P Woods, Liberty S Hamilton, Kristi Clark, Owen Phillips, David W Shattuck, Robert F Asarnow, Arthur W Toga, and Keith H Nuechterlein. Mean diffusivity: a biomarker for csf-related disease and genetic liability effects in schizophrenia. Psychiatry Research: Neuroimaging, 171(1):20-32, 2009.

[142] Cheng Guan Koay, Lin-Ching Chang, John D Carew, Carlo Pierpaoli, and Peter J Basser. A unifying theoretical and algorithmic framework for least squares methods of estimation in diffusion tensor imaging. Journal of Magnetic Resonance, 182(1):115-125, 2006.

[143] Alexander Lerner, Monique A Mogensen, Paul E Kim, Mark S Shiroishi, Darryl H Hwang, and Meng Law. Clinical applications of diffusion tensor imaging. World neurosurgery, 2013.

[144] Denis Le Bihan, Jean-François Mangin, Cyril Poupon, Chris A Clark, Sabina Pappata, Nicolas Molko, and Hughes Chabriat. Diffusion tensor imaging: concepts and applications. Journal of magnetic resonance imaging, 13(4):534-546, 2001.

[145] Sheng-Kwei Song, Jun Yoshino, Tuan Q Le, Shiow-Jiuan Lin, Shu-Wei Sun, Anne H Cross, and Regina C Armstrong. Demyelination increases radial diffusivity in corpus callosum of mouse brain. Neuroimage, 26(1):132-140, 2005.

[146] Paolo GP Nucifora, Ragini Verma, S-K Lee, and Elias R Melhem. Diffusion-tensor MR imaging and tractography: exploring brain microstructure and connectivity. $R a$ diology, 245(2):367, 2007.

[147] Peter J Basser and Derek K Jones. Diffusion-tensor mri: theory, experimental design and data analysis-a technical review. NMR in Biomedicine, 15(7-8):456-467, 2002.

[148] TEJ Behrens, MW Woolrich, M Jenkinson, H Johansen-Berg, RG Nunes, S Clare, PM Matthews, JM Brady, and SM Smith. Characterization and propagation of uncertainty in diffusion-weighted $\mathrm{mr}$ imaging. Magnetic resonance in medicine, 50(5):1077-1088, 2003.

[149] Brittany G Travers, Nagesh Adluru, Chad Ennis, Do PM Tromp, Dan Destiche, Sam Doran, Erin D Bigler, Nicholas Lange, Janet E Lainhart, and Andrew L Alexander. Diffusion tensor imaging in autism spectrum disorder: a review. Autism Research, 5(5):289-313, 2012.

[150] Manuel F Casanova, Daniel P Buxhoeveden, and Caroline Brown. Clinical and macroscopic correlates of minicolumnar pathology in autism. Journal of Child Neurology, 17(9):692-695, 2002.

[151] Manuel F Casanova, Imke AJ van Kooten, Andrew E Switala, Herman van Engeland, Helmut Heinsen, Harry WM Steinbusch, Patrick R Hof, Juan Trippe, Janet Stone, and Christoph Schmitz. Minicolumnar abnormalities in autism. Acta neuropathologica, 112(3):287-303, 2006. 
[152] Sarah E Schipul, Timothy A Keller, and Marcel Adam Just. Inter-regional brain communication and its disturbance in autism. Front Syst Neurosci, 5(10):7, 2011.

[153] Jason J Wolff, Hongbin Gu, Guido Gerig, Jed T Elison, Martin Styner, Sylvain Gouttard, Kelly N Botteron, Stephen R Dager, Geraldine Dawson, Annette M Estes, Alan C Evans, Heather C Hazlett, P Kostopoulos, Robert C McKinstry, Sarah J Paterosn, Robert T Schultz, L Zwaigenbaum, and J Piven. Differences in white matter fiber tract development present from 6 to 24 months in infants with autism. American Journal of Psychiatry, 169(6):589-600, 2012.

[154] C Cheung, SE Chua, V Cheung, PL Khong, KS Tai, TKW Wong, TP Ho, and GM McAlonan. White matter fractional anisotrophy differences and correlates of diagnostic symptoms in autism. Journal of Child Psychology and Psychiatry, 50(9):1102-1112, 2009.

[155] Xiaoyan Ke, Tianyu Tang, Shanshan Hong, Yueyue Hang, Bing Zou, Huiguo Li, Zhenyu Zhou, Zongcai Ruan, Zuhong Lu, Guotai Tao, and Yijun Liu. White matter impairments in autism, evidence from voxel-based morphometry and diffusion tensor imaging. Brain research, 1265:171-177, 2009.

[156] Naama Barnea-Goraly, Linda J Lotspeich, and Allan L Reiss. Similar white matter aberrations in children with autism and their unaffected siblings: a diffusion tensor imaging study using tract-based spatial statistics. Archives of general psychiatry, 67(10):1052-1060, 2010.

[157] Luise Poustka, Christine Jennen-Steinmetz, Romy Henze, Kilian Vomstein, Johann Haffner, and Bram Sieltjes. Fronto-temporal disconnectivity and symptom severity in children with autism spectrum disorder. World Journal of Biological Psychiatry, 13(4):269-280, 2012.

[158] Naama Barnea-Goraly, Hower Kwon, Vinod Menon, Stephan Eliez, Linda Lotspeich, and Allan L Reiss. White matter structure in autism: preliminary evidence from diffusion tensor imaging. Biological psychiatry, 55(3):323-326, 2004.

[159] Timothy A Keller, Rajesh K Kana, and Marcel Adam Just. A developmental study of the structural integrity of white matter in autism. Neuroreport, 18(1):23-27, 2007.

[160] Jee Eun Lee, Moo K Chung, Mariana Lazar, Molly B DuBray, Jinsuh Kim, Erin D Bigler, Janet E Lainhart, and Andrew L Alexander. A study of diffusion tensor imaging by tissue-specific, smoothing-compensated voxel-based analysis. Neuroimage, 44(3):870-883, 2009.

[161] Madoka Noriuchi, Yoshiaki Kikuchi, Takashi Yoshiura, Ryutaro Kira, Hiroshi Shigeto, Toshiro Hara, Shozo Tobimatsu, and Yoko Kamio. Altered white matter fractional anisotropy and social impairment in children with autism spectrum disorder. Brain research, 1362:141-149, 2010.

[162] Roger J Jou, Andrea P Jackowski, Xenophon Papademetris, Nallakkandi Rajeevan, Lawrence H Staib, and Fred R Volkmar. Diffusion tensor imaging in autism spectrum disorders: preliminary evidence of abnormal neural connectivity. Australian and New Zealand Journal of Psychiatry, 45(2):153-162, 2011. 
[163] Wouter B Groen, Jan K Buitelaar, Rutger J Van Der Gaag, and Marcel P Zwiers. Pervasive microstructural abnormalities in autism: a dti study. Journal of Psychiatry \& Neuroscience: JPN, 36(1):32, 2011.

[164] Oswald JN Bloemen, Quinton Deeley, Fred Sundram, Eileen M Daly, Gareth J Barker, Derek K Jones, Therese AMJ van Amelsvoort, Nicole Schmitz, Dene Robertson, Kieran C Murphy, and Declan GM Murphy. White matter integrity in asperger syndrome: a preliminary diffusion tensor magnetic resonance imaging study in adults. Autism Research, 3(5):203-213, 2010.

[165] Matteo Pardini, Maurizio Elia, Francesco G Garaci, Silvia Guida, Filadelfo Coniglione, Frank Krueger, Francesca Benassi, and Leonardo Emberti Gialloreti. Long-term cognitive and behavioral therapies, combined with augmentative communication, are related to uncinate fasciculus integrity in autism. Journal of autism and developmental disorders, 42(4):585-592, 2012.

[166] Stephen M Smith, Mark Jenkinson, Heidi Johansen-Berg, Daniel Rueckert, Thomas E Nichols, Clare E Mackay, Kate E Watkins, Olga Ciccarelli, M Zaheer Cader, Paul M Matthews, and Timothy EJ Behrens. Tract-based spatial statistics: voxelwise analysis of multi-subject diffusion data. Neuroimage, 31(4):1487-1505, 2006.

[167] Ajay Kumar, Senthil K Sundaram, Lalitha Sivaswamy, Michael E Behen, Malek I Makki, Joel Ager, James Janisse, Harry T Chugani, and Diane C Chugani. Alterations in frontal lobe tracts and corpus callosum in young children with autism spectrum disorder. Cerebral cortex, 20(9):2103-2113, 2010.

[168] Maya Weinstein, Liat Ben-Sira, Yonata Levy, Ditza A Zachor, Esti Ben Itzhak, Moran Artzi, Ricardo Tarrasch, Perla M Eksteine, Talma Hendler, and Dafna Ben Bashat. Abnormal white matter integrity in young children with autism. Human brain mapping, 32(4):534-543, 2011.

[169] Keun-Ah Cheon, Young-Shin Kim, Se-Hong Oh, Sung-Yeon Park, Hyo-Woon Yoon, John Herrington, Aarti Nair, Yun-Joo Koh, Dong-Pyo Jang, Young-Bo Kim, BL Leventhal, Zang-Hee Cho, F Xavier Castellanos, and Robert T Schultz. Involvement of the anterior thalamic radiation in boys with high functioning autism spectrum disorders: a diffusion tensor imaging study. Brain research, 1417:77-86, 2011.

[170] RJ Jou, N Mateljevic, MD Kaiser, DR Sugrue, FR Volkmar, and KA Pelphrey. Structural neural phenotype of autism: preliminary evidence from a diffusion tensor imaging study using tract-based spatial statistics. American Journal of Neuroradiology, 32(9):1607-1613, 2011.

[171] Lindsay Walker, Marta Gozzi, Rhoshel Lenroot, Audrey Thurm, Babak Behseta, Susan Swedo, and Carlo Pierpaoli. Diffusion tensor imaging in young children with autism: biological effects and potential confounds. Biological psychiatry, 72(12):1043-1051, 2012.

[172] Lucia Billeci, Sara Calderoni, Michela Tosetti, Marco Catani, and Filippo Muratori. White matter connectivity in children with autism spectrum disorders: a tract-based spatial statistics study. BMC neurology, 12(1):148, 2012. 
[173] Chérif P Sahyoun, John W Belliveau, and Maria Mody. White matter integrity and pictorial reasoning in high-functioning children with autism. Brain and cognition, 73(3):180-188, 2010.

[174] Stephanie H Ameis, Jin Fan, Conrad Rockel, Aristotle N Voineskos, Nancy J Lobaugh, Latha Soorya, A Ting Wang, Eric Hollander, and Evdokia Anagnostou. Impaired structural connectivity of socio-emotional circuits in autism spectrum disorders: a diffusion tensor imaging study. PLoS One, 6(11):e28044, 2011.

[175] Dinesh K Shukla, Brandon Keehn, Daren M Smylie, and Ralph-Axel Müller. Microstructural abnormalities of short-distance white matter tracts in autism spectrum disorder. Neuropsychologia, 49(5):1378-1382, 2011.

[176] Dinesh K Shukla, Brandon Keehn, and Ralph-Axel Müller. Tract-specific analyses of diffusion tensor imaging show widespread white matter compromise in autism spectrum disorder. Journal of Child Psychology and Psychiatry, 52(3):286-295, 2011.

[177] Michaela K Bode, Marja-Leena Mattila, Vesa Kiviniemi, Jukka Rahko, Irma Moilanen, Hanna Ebeling, Osmo Tervonen, and Juha Nikkinen. White matter in autism spectrum disorders-evidence of impaired fiber formation. Acta Radiologica, 52(10):1169-1174, 2011.

[178] Rajesh K Kana, Lauren E Libero, Christi P Hu, Hrishikesh D Deshpande, and Jeffrey S Colburn. Functional brain networks and white matter underlying theory-ofmind in autism. Social cognitive and affective neuroscience, 9(1):98-105, 2014.

[179] Thomas John Perkins, Mark Andrew Stokes, Jane Anne McGillivray, Alexander Julien Mussap, Ivanna Anne Cox, Jerome Joseph Maller, and Richard Garth Bittar. Increased left hemisphere impairment in high-functioning autism: A tract based spatial statistics study. Psychiatry Research: Neuroimaging, 224(2):119-123, 2014.

[180] Dafna Ben Bashat, Vered Kronfeld-Duenias, Ditza A Zachor, Perla M Ekstein, Talma Hendler, Ricardo Tarrasch, Ariela Even, Yonata Levy, and Liat Ben Sira. Accelerated maturation of white matter in young children with autism: a high $b$ value dwi study. Neuroimage, 37(1):40-47, 2007.

[181] Adriana Rocha Brito, Marcio Moacyr Vasconcelos, Romeu Cortes Domingues, Hygino da Cruz Jr, Luiz Celso, Leise de Souza Rodrigues, Emerson L Gasparetto, and Carlos Adolfo B Pinto Calçada. Diffusion tensor imaging findings in school-aged autistic children. Journal of Neuroimaging, 19(4):337-343, 2009.

[182] Lalitha Sivaswamy, Ajay Kumar, Deepa Rajan, Michael Behen, Otto Muzik, Diane Chugani, and Harry Chugani. A diffusion tensor imaging study of the cerebellar pathways in children with autism spectrum disorder. Journal of child neurology, 2010.

[183] Emma G Duerden, Dallas Card, S Wendy Roberts, Kathleen M Mak-Fan, M Mallar Chakravarty, Jason P Lerch, and Margot J Taylor. Self-injurious behaviours are associated with alterations in the somatosensory system in children with autism spectrum disorder. Brain Structure and Function, 219(4):1251-1261, 2014. 
[184] Daniel Peterson, Rajneesh Mahajan, Deana Crocetti, Amanda Mejia, and Stewart Mostofsky. Left-hemispheric microstructural abnormalities in children with highfunctioning autism spectrum disorder. Autism Research, 2014.

[185] Jee Eun Lee, Erin D Bigler, Andrew L Alexander, Mariana Lazar, Molly B DuBray, Moo K Chung, Michael Johnson, Jubel Morgan, Judith N Miller, William M McMahon, Jeffrey Lu, Eun-Kee Jeong, and Janet E Lainhart. Diffusion tensor imaging of white matter in the superior temporal gyrus and temporal stem in autism. Neuroscience Letters, 424(2):127-132, 2007.

[186] Dinesh K Shukla, Brandon Keehn, Alan J Lincoln, and Ralph-Axel Müller. White matter compromise of callosal and subcortical fiber tracts in children with autism spectrum disorder: a diffusion tensor imaging study. Journal of the American Academy of Child \& Adolescent Psychiatry, 49(12):1269-1278, 2010.

[187] Katharine N Thakkar, Frida E Polli, Robert M Joseph, David S Tuch, Nouchine Hadjikhani, Jason JS Barton, and Dara S Manoach. Response monitoring, repetitive behaviour and anterior cingulate abnormalities in autism spectrum disorders (asd). Brain, 131(9):2464-2478, 2008.

[188] FD Beacher, Ludovico Minati, Simon Baron-Cohen, MV Lombardo, M-C Lai, MA Gray, NA Harrison, and HD Critchley. Autism attenuates sex differences in brain structure: a combined voxel-based morphometry and diffusion tensor imaging study. American Journal of Neuroradiology, 33(1):83-89, 2012.

[189] Jed T Elison, Sarah J Paterson, Jason J Wolff, J Steven Reznick, Noah J Sasson, Hongbin Gu, Kelly N Botteron, Stephen R Dager, Annette M Estes, Alan C Evans, Guido Gerig, Heather C Hazlett, Robert T Schultz, Martin Styner, Lonnie Zwaigenbaum, and Joseph Piven. White matter microstructure and atypical visual orienting in 7-month-olds at risk for autism. American Journal of Psychiatry, 170(8):899-908, 2014.

[190] Senthil K Sundaram, Ajay Kumar, Malek I Makki, Michael E Behen, Harry T Chugani, and Diane C Chugani. Diffusion tensor imaging of frontal lobe in autism spectrum disorder. Cerebral cortex, 18(11):2659-2665, 2008.

[191] Shanshan Hong, Xiaoyan Ke, Tianyu Tang, Yueyue Hang, Kangkang Chu, Haiqing Huang, Zongcai Ruan, Zuhong Lu, Guotai Tao, and Yijun Liu. Detecting abnormalities of corpus callosum connectivity in autism using magnetic resonance imaging and diffusion tensor tractography. Psychiatry Research: Neuroimaging, 194(3):333339, 2011.

[192] J-W Jeong, AK Kumar, Senthil K Sundaram, Harry T Chugani, and Diane C Chugani. Sharp curvature of frontal lobe white matter pathways in children with autism spectrum disorders: tract-based morphometry analysis. American Journal of Neuroradiology, 32(9):1600-1606, 2011.

[193] Catherine Y Wan, Sarah Marchina, Andrea Norton, and Gottfried Schlaug. Atypical hemispheric asymmetry in the arcuate fasciculus of completely nonverbal children with autism. Annals of the New York Academy of Sciences, 1252(1):332-337, 2012. 
[194] LM Nagae, DM Zarnow, L Blaskey, J Dell, SY Khan, S Qasmieh, SE Levy, and TPL Roberts. Elevated mean diffusivity in the left hemisphere superior longitudinal fasciculus in autism spectrum disorders increases with more profound language impairment. American Journal of Neuroradiology, 33(9):1720-1725, 2012.

[195] Grace Lai, Spiro P Pantazatos, Harry Schneider, and Joy Hirsch. Neural systems for speech and song in autism. Brain, 135(3):961-975, 2012.

[196] Brian D Mills, Janie Lai, Timothy T Brown, Matthew Erhart, Eric Halgren, Judy Reilly, Anders Dale, Mark Appelbaum, and Pamela Moses. White matter microstructure correlates of narrative production in typically developing children and children with high functioning autism. Neuropsychologia, 51(10):1933-1941, 2013.

[197] Robert M Joseph, Zachary Fricker, Angela Fenoglio, Kristen A Lindgren, Tracey A Knaus, and Helen Tager-Flusberg. Structural asymmetries of language-related gray and white matter and their relationship to language function in young children with asd. Brain imaging and behavior, 8(1):60-72, 2014.

[198] Tracey A Knaus, Andrew M Silver, Meaghan Kennedy, Kristen A Lindgren, Kelli C Dominick, Jeremy Siegel, and Helen Tager-Flusberg. Language laterality in autism spectrum disorder and typical controls: a functional, volumetric, and diffusion tensor mri study. Brain and language, 112(2):113-120, 2010.

[199] P Thomas Fletcher, Ross T Whitaker, Ran Tao, Molly B DuBray, Alyson Froehlich, Caitlin Ravichandran, Andrew L Alexander, Erin D Bigler, Nicholas Lange, and Janet E Lainhart. Microstructural connectivity of the arcuate fasciculus in adolescents with high-functioning autism. Neuroimage, 51(3):1117-1125, 2010.

[200] Judith S Verhoeven, Nathalie Rommel, Elena Prodi, Alexander Leemans, Inge Zink, Ellen Vandewalle, Ilse Noens, Johan Wagemans, Jean Steyaert, Bart Boets, A Van de Winckel, Paul De Cock, Lieven Lagae, and Stefan Sunaert. Is there a common neuroanatomical substrate of language deficit between autism spectrum disorder and specific language impairment? Cerebral Cortex, 22(10):2263-2271, 2012.

[201] Yu-Chun Lo, Wei-Tsuen Soong, Susan Shur-Fen Gau, Yu-Yu Wu, Meng-Chuan Lai, Fang-Cheng Yeh, Wen-Yang Chiang, Li-Wei Kuo, Fu-Shan Jaw, and Wen-Yih Isaac Tseng. The loss of asymmetry and reduced interhemispheric connectivity in adolescents with autism: a study using diffusion spectrum imaging tractography. Psychiatry Research: Neuroimaging, 192(1):60-66, 2011.

[202] Stephanie H Ameis, Jin Fan, Conrad Rockel, Latha Soorya, A Ting Wang, and Evdokia Anagnostou. Altered cingulum bundle microstructure in autism spectrum disorder. Acta neuropsychiatrica, 25(05):275-282, 2013.

[203] Aarti Nair, Jeffrey M Treiber, Dinesh K Shukla, Patricia Shih, and Ralph-Axel Müller. Impaired thalamocortical connectivity in autism spectrum disorder: a study of functional and anatomical connectivity. Brain, 136(6):1942-1955, 2013.

[204] Toshikazu Ikuta, Keith M Shafritz, Joel Bregman, Bart D Peters, Patricia Gruner, Anil K Malhotra, and Philip R Szeszko. Abnormal cingulum bundle development in autism: a probabilistic tractography study. Psychiatry Research: Neuroimaging, 221(1):63-68, 2014. 
[205] Marco Catani, Derek K Jones, Eileen Daly, Nitzia Embiricos, Quinton Deeley, Luca Pugliese, Sarah Curran, Dene Robertson, and Declan GM Murphy. Altered cerebellar feedback projections in asperger syndrome. Neuroimage, 41(4):1184-1191, 2008.

[206] Thomas E Conturo, Diane L Williams, Charles D Smith, Eren Gultepe, Erbil Akbudak, and Nancy J Minshew. Neuronal fiber pathway abnormalities in autism: an initial mri diffusion tensor tracking study of hippocampo-fusiform and amygdalofusiform pathways. Journal of the International Neuropsychological Society, 14(06):933-946, 2008.

[207] Luca Pugliese, Marco Catani, Stephanie Ameis, Flavio Dell'Acqua, Michel Thiebaut de Schotten, Clodagh Murphy, Dene Robertson, Quinton Deeley, Eileen Daly, and Declan GM Murphy. The anatomy of extended limbic pathways in asperger syndrome: a preliminary diffusion tensor imaging tractography study. Neuroimage, 47(2):427-434, 2009.

[208] Cibu Thomas, Kate Humphreys, Kwan-Jin Jung, Nancy Minshew, and Marlene Behrmann. The anatomy of the callosal and visual-association pathways in highfunctioning autism: a dti tractography study. Cortex, 47(7):863-873, 2011.

[209] Marieke Langen, Alexander Leemans, Patrick Johnston, Christine Ecker, Eileen Daly, Clodagh M Murphy, Flavio dellAcqua, Sarah Durston, Declan GM Murphy, and AIMS Consortium. Fronto-striatal circuitry and inhibitory control in autism: findings from diffusion tensor imaging tractography. Cortex, 48(2):183-193, 2012.

[210] John D Lewis, Rebecca J Theilmann, Vladimir Fonov, Pierre Bellec, Alan Lincoln, Alan C Evans, and Jeanne Townsend. Callosal fiber length and interhemispheric connectivity in adults with autism: brain overgrowth and underconnectivity. Human brain mapping, 34(7):1685-1695, 2013.

[211] Hidetaka Arimura, Taiki Magome, Yasuo Yamashita, and Daisuke Yamamoto. Computer-aided diagnosis systems for brain diseases in magnetic resonance images. Algorithms, 2(3):925-952, 2009.

[212] Madhura Ingalhalikar, Drew Parker, Luke Bloy, Timothy PL Roberts, and Ragini Verma. Diffusion based abnormality markers of pathology: toward learned diagnostic prediction of asd. Neuroimage, 57(3):918-927, 2011.

[213] Nagesh Adluru, Chris Hinrichs, Moo K Chung, J-E Lee, Vikas Singh, Erin D Bigler, Nicholas Lange, Janet E Lainhart, and Andrew L Alexander. Classification in dti using shapes of white matter tracts. In Engineering in Medicine and Biology Society, 2009. EMBC 2009. Annual International Conference of the IEEE, pages 2719-2722. IEEE, 2009.

[214] Serge Belongie, Jitendra Malik, and Jan Puzicha. Shape matching and object recognition using shape contexts. Pattern Analysis and Machine Intelligence, IEEE Transactions on, 24(4):509-522, 2002.

[215] Nicholas Lange, Molly B DuBray, Jee Eun Lee, Michael P Froimowitz, Alyson Froehlich, Nagesh Adluru, Brad Wright, Caitlin Ravichandran, P Thomas Fletcher, Erin D Bigler, Andrew L Alexander, and Janet E Lainhart. Atypical diffusion tensor hemispheric asymmetry in autism. Autism research, 3(6):350-358, 2010. 
[216] Greg Allen and Eric Courchesne. Differential effects of developmental cerebellar abnormality on cognitive and motor functions in the cerebellum: an fmri study of autism. American Journal of Psychiatry, 160(2):262-273, 2003.

[217] Greg Allen, Ralph-Axel Müller, and Eric Courchesne. Cerebellar function in autism: functional magnetic resonance image activation during a simple motor task. Biological psychiatry, 56(4):269-278, 2004.

[218] Ralph-Axel Müller, Cassandra Cauich, Miguel A Rubio, Akiko Mizuno, and Eric Courchesne. Abnormal activity patterns in premotor cortex during sequence learning in autistic patients. Biological psychiatry, 56(5):323-332, 2004.

[219] Ralph-Axel Müller, Natalia Kleinhans, Nobuko Kemmotsu, Karen Pierce, and Eric Courchesne. Abnormal variability and distribution of functional maps in autism: an fmri study of visuomotor learning. American Journal of Psychiatry, 160(10):18471862, 2003.

[220] Ralph-Axel Müller, Karen Pierce, Josiah B Ambrose, Greg Allen, and Eric Courchesne. Atypical patterns of cerebral motor activation in autism: a functional magnetic resonance study. Biological psychiatry, 49(8):665-676, 2001.

[221] Tom J Perkins, Richard G Bittar, Jane A McGillivray, Ivanna I Cox, and Mark A Stokes. Increased premotor cortex activation in high functioning autism during action observation. Journal of Clinical Neuroscience, 22(4):664-669, 2015.

[222] S Bölte, D Hubl, T Dierks, M Holtmann, and F Poustka. An fmri-study of locally oriented perception in autism: altered early visual processing of the block design test. Journal of Neural Transmission, 115(3):545-552, 2008.

[223] Brandon Keehn, Laurie Brenner, Erica Palmer, Alan J Lincoln, and Ralph-Axel Mueller. Functional brain organization for visual search in asd. Journal of the International Neuropsychological Society, 14(06):990-1003, 2008.

[224] Zina M Manjaly, Nicole Bruning, Susanne Neufang, Klaas E Stephan, Sarah Brieber, John C Marshall, Inge Kamp-Becker, Helmut Remschmidt, Beate HerpertzDahlmann, Kerstin Konrad, et al. Neurophysiological correlates of relatively enhanced local visual search in autistic adolescents. Neuroimage, 35(1):283-291, 2007.

[225] Chérif P Sahyoun, Isabelle Soulieres, John W Belliveau, Laurent Mottron, and Maria Mody. Cognitive differences in pictorial reasoning between high-functioning autism and aspergers syndrome. Journal of Autism and Developmental Disorders, 39(7):1014-1023, 2009.

[226] Philip S Lee, Jennifer Foss-Feig, Joshua G Henderson, Lauren E Kenworthy, Lisa Gilotty, William D Gaillard, and Chandan J Vaidya. Atypical neural substrates of embedded figures task performance in children with autism spectrum disorder. Neuroimage, 38(1):184-193, 2007.

[227] Yukari Takarae, Nancy J Minshew, Beatriz Luna, and John A Sweeney. Atypical involvement of frontostriatal systems during sensorimotor control in autism. Psychiatry Research: Neuroimaging, 156(2):117-127, 2007. 
[228] H Clery, F Andersson, F Bonnet-Brilhault, A Philippe, B Wicker, and M Gomot. fmri investigation of visual change detection in adults with autism. NeuroImage: Clinical, 2:303-312, 2013.

[229] Gabriel S Dichter and Aysenil Belger. Social stimuli interfere with cognitive control in autism. Neuroimage, 35(3):1219-1230, 2007.

[230] Sam J Gilbert, Geoffrey Bird, Rachel Brindley, Christopher D Frith, and Paul W Burgess. Atypical recruitment of medial prefrontal cortex in autism spectrum disorders: An fmri study of two executive function tasks. Neuropsychologia, 46(9):22812291, 2008.

[231] Marcel Adam Just, Vladimir L Cherkassky, Timothy A Keller, Rajesh K Kana, and Nancy J Minshew. Functional and anatomical cortical underconnectivity in autism: evidence from an fmri study of an executive function task and corpus callosum morphometry. Cerebral cortex, 17(4):951-961, 2007.

[232] Rajesh K Kana, Timothy A Keller, Nancy J Minshew, and Marcel Adam Just. Inhibitory control in high-functioning autism: decreased activation and underconnectivity in inhibition networks. Biological psychiatry, 62(3):198-206, 2007.

[233] Hideya Koshino, Rajesh K Kana, Timothy A Keller, Vladimir L Cherkassky, Nancy J Minshew, and Marcel Adam Just. fmri investigation of working memory for faces in autism: visual coding and underconnectivity with frontal areas. Cerebral cortex, 18(2):289-300, 2008.

[234] Philip S Lee, Benjamin E Yerys, Anne Della Rosa, Jennifer Foss-Feig, Kelly Anne Barnes, Joette D James, John VanMeter, Chandan J Vaidya, William D Gaillard, and Lauren E Kenworthy. Functional connectivity of the inferior frontal cortex changes with age in children with autism spectrum disorders: a fcmri study of response inhibition. Cerebral Cortex, 19(8):1787-1794, 2009.

[235] Nicole Schmitz, Katya Rubia, Therese Van Amelsvoort, Eileen Daly, Anna Smith, and Declan GM Murphy. Neural correlates of reward in autism. The British Journal of Psychiatry, 192(1):19-24, 2008.

[236] Keith M Shafritz, Gabriel S Dichter, Grace T Baranek, and Aysenil Belger. The neural circuitry mediating shifts in behavioral response and cognitive set in autism. Biological psychiatry, 63(10):974-980, 2008.

[237] Marie Gomot, Frédéric A Bernard, Matthew H Davis, Matthew K Belmonte, Chris Ashwin, Edward T Bullmore, and Simon Baron-Cohen. Change detection in children with autism: an auditory event-related fmri study. Neuroimage, 29(2):475-484, 2006.

[238] Marie Gomot, Matthew K Belmonte, Edward T Bullmore, Frédéric A Bernard, and Simon Baron-Cohen. Brain hyper-reactivity to auditory novel targets in children with high-functioning autism. Brain, 131(9):2479-2488, 2008.

[239] Tracey A Knaus, Andrew M Silver, Kristen A Lindgren, Nouchine Hadjikhani, and Helen Tager-Flusberg. fmri activation during a language task in adolescents with asd. Journal of the International Neuropsychological Society, 14(06):967-979, 2008. 
[240] Elizabeth Redcay and Eric Courchesne. Deviant functional magnetic resonance imaging patterns of brain activity to speech in 2-3-year-old children with autism spectrum disorder. Biological psychiatry, 64(7):589-598, 2008.

[241] A Ting Wang, Susan S Lee, Marian Sigman, and Mirella Dapretto. Reading affect in the face and voice: neural correlates of interpreting communicative intent in children and adolescents with autism spectrum disorders. Archives of general psychiatry, 64(6):698-708, 2007.

[242] A Ting Wang, Susan S Lee, Marian Sigman, and Mirella Dapretto. Neural basis of irony comprehension in children with autism: the role of prosody and context. Brain, 129(4):932-943, 2006.

[243] Michael V Lombardo, Karen Pierce, Lisa T Eyler, Cindy Carter Barnes, Clelia Ahrens-Barbeau, Stephanie Solso, Kathleen Campbell, and Eric Courchesne. Different functional neural substrates for good and poor language outcome in autism. Neuron, 86(2):567-577, 2015.

[244] Michael S Gaffrey, Natalia M Kleinhans, Frank Haist, Natacha Akshoomoff, Ashley Campbell, Eric Courchesne, and Ralph-Axel Müller. A typical participation of visual cortex during word processing in autism: An fmri study of semantic decision. Neuropsychologia, 45(8):1672-1684, 2007.

[245] Hélène Gervais, Pascal Belin, Nathalie Boddaert, Marion Leboyer, Arnaud Coez, Ignacio Sfaello, Catherine Barthélémy, Francis Brunelle, Yves Samson, and Mônica Zilbovicius. Abnormal cortical voice processing in autism. Nature neuroscience, 7(8):801-802, 2004.

[246] Gordon J Harris, Christopher F Chabris, Jill Clark, Trinity Urban, Itzhak Aharon, Shelley Steele, Lauren McGrath, Karen Condouris, and Helen Tager-Flusberg. Brain activation during semantic processing in autism spectrum disorders via functional magnetic resonance imaging. Brain and cognition, 61(1):54-68, 2006.

[247] Rajesh K Kana, Timothy A Keller, Vladimir L Cherkassky, Nancy J Minshew, and Marcel Adam Just. Sentence comprehension in autism: thinking in pictures with decreased functional connectivity. Brain, 129(9):2484-2493, 2006.

[248] Natalia M Kleinhans, Ralph-Axel Müller, David N Cohen, and Eric Courchesne. Atypical functional lateralization of language in autism spectrum disorders. Brain research, 1221:115-125, 2008.

[249] Robert A Mason, Diane L Williams, Rajesh K Kana, Nancy Minshew, and Marcel Adam Just. Theory of mind disruption and recruitment of the right hemisphere during narrative comprehension in autism. Neuropsychologia, 46(1):269-280, 2008.

[250] Lisa B Wilson, Jason R Tregellas, Erin Slason, Bryce E Pasko, Susan Hepburn, and Donald C Rojas. Phonological processing in first-degree relatives of individuals with autism: An fmri study. Human brain mapping, 34(6):1447-1463, 2013.

[251] Susan Y Bookheimer, A Ting Wang, Ashley Scott, Marian Sigman, and Mirella Dapretto. Frontal contributions to face processing differences in autism: evidence from fmri of inverted face processing. Journal of the International Neuropsychological Society, 14(06):922-932, 2008. 
[252] Blythe A Corbett, Vanessa Carmean, Susan Ravizza, Carter Wendelken, Melissa L Henry, Cameron Carter, and Susan M Rivera. A functional and structural study of emotion and face processing in children with autism. Psychiatry Research: Neuroimaging, 173(3):196-205, 2009.

[253] Nouchine Hadjikhani, Robert M Joseph, Josh Snyder, and Helen Tager-Flusberg. Abnormal activation of the social brain during face perception in autism. Human brain mapping, 28(5):441-449, 2007.

[254] Kevin A Pelphrey, James P Morris, Gregory McCarthy, and Kevin S LaBar. Perception of dynamic changes in facial affect and identity in autism. Social cognitive and affective neuroscience, 2007.

[255] Chris Ashwin, Simon Baron-Cohen, Sally Wheelwright, Michelle ORiordan, and Edward T Bullmore. Differential activation of the amygdala and the social brainduring fearful face-processing in asperger syndrome. Neuropsychologia, 45(1):2-14, 2007.

[256] Quinton Deeley, Eileen M Daly, Simon Surguladze, Lisa Page, Fiona Toal, Dene Robertson, Sarah Curran, Vincent Giampietro, Marc Seal, Michael J Brammer, et al. An event related functional magnetic resonance imaging study of facial emotion processing in asperger syndrome. Biological psychiatry, 62(3):207-217, 2007.

[257] Ellen Greimel, Martin Schulte-Rüther, Tilo Kircher, Inge Kamp-Becker, Helmut Remschmidt, Gereon R Fink, Beate Herpertz-Dahlmann, and Kerstin Konrad. Neural mechanisms of empathy in adolescents with autism spectrum disorder and their fathers. Neuroimage, 49(1):1055-1065, 2010.

[258] Nouchine Hadjikhani, Robert M Joseph, Dara S Manoach, Paulami Naik, Josh Snyder, Kelli Dominick, Rick Hoge, Jan Van den Stock, Helen Tager Flusberg, and Beatrice De Gelder. Body expressions of emotion do not trigger fear contagion in autism spectrum disorder. Social cognitive and affective neuroscience, page nsn038, 2009.

[259] Katherine A Loveland, Joel L Steinberg, Deborah A Pearson, Rosleen Mansour, and Stacy Reddoch. Judgments of auditoryvisual affective congruence in adolescents with and without autism: A pilot study of a new task using fmri. Perceptual and motor skills, 107(2):557-575, 2008.

[260] Bruno Wicker, Pierre Fonlupt, Bénédicte Hubert, Carole Tardif, Bruno Gepner, and Christine Deruelle. Abnormal cerebral effective connectivity during explicit emotional processing in adults with autism spectrum disorder. Social cognitive and affective neuroscience, 3(2):135-143, 2008.

[261] Natalia M Kleinhans, Todd Richards, Jessica Greenson, Geraldine Dawson, and Elizabeth Aylward. Altered dynamics of the fmri response to faces in individuals with autism. Journal of autism and developmental disorders, 46(1):232-241, 2016.

[262] Geoffrey Bird, Caroline Catmur, Giorgia Silani, Chris Frith, and Uta Frith. Attention does not modulate neural responses to social stimuli in autism spectrum disorders. Neuroimage, 31(4):1614-1624, 2006. 
[263] Gabriel S Dichter and Aysenil Belger. Atypical modulation of cognitive control by arousal in autism. Psychiatry Research: Neuroimaging, 164(3):185-197, 2008.

[264] Christine M Freitag, Carsten Konrad, Melanie Häberlen, Christina Kleser, Alexander von Gontard, Wolfgang Reith, Nikolaus F Troje, and Christoph Krick. Perception of biological motion in autism spectrum disorders. Neuropsychologia, 46(5):1480-1494, 2008.

[265] J Grezes, B Wicker, S Berthoz, and B De Gelder. A failure to grasp the affective meaning of actions in autism spectrum disorder subjects. Neuropsychologia, 47(8):1816-1825, 2009.

[266] Kate Humphreys, Uri Hasson, Galia Avidan, Nancy Minshew, and Marlene Behrmann. Cortical patterns of category-selective activation for faces, places and objects in adults with autism. Autism Research, 1(1):52-63, 2008.

[267] Pearl H Chiu, M Amin Kayali, Kenneth T Kishida, Damon Tomlin, Laura G Klinger, Mark R Klinger, and P Read Montague. Self responses along cingulate cortex reveal quantitative neural phenotype for high-functioning autism. Neuron, 57(3):463-473, 2008.

[268] Mirella Dapretto, Mari S Davies, Jennifer H Pfeifer, Ashley A Scott, Marian Sigman, Susan Y Bookheimer, and Marco Iacoboni. Understanding emotions in others: mirror neuron dysfunction in children with autism spectrum disorders. Nature neuroscience, 9(1):28-30, 2006.

[269] Lucina Q Uddin, Mari S Davies, Ashley A Scott, Eran Zaidel, Susan Y Bookheimer, Marco Iacoboni, and Mirella Dapretto. Neural basis of self and other representation in autism: an fmri study of self-face recognition. PloS one, 3(10):e3526, 2008.

[270] Justin HG Williams, Gordon D Waiter, Anne Gilchrist, David I Perrett, Alison D Murray, and Andrew Whiten. Neural mechanisms of imitation and mirror neuronfunctioning in autistic spectrum disorder. Neuropsychologia, 44(4):610-621, 2006.

[271] Sam J Gilbert, Julia DI Meuwese, Karren J Towgood, Christopher D Frith, and Paul W Burgess. Abnormal functional specialization within medial prefrontal cortex in high-functioning autism: a multi-voxel similarity analysis. Brain, page awn365, 2009.

[272] Rajesh K Kana, Timothy A Keller, Vladimir L Cherkassky, Nancy J Minshew, and Marcel Adam Just. Atypical frontal-posterior synchronization of theory of mind regions in autism during mental state attribution. Social neuroscience, 4(2):135$152,2009$.

[273] Giorgia Silani, Geoffrey Bird, Rachel Brindley, Tania Singer, Chris Frith, and Uta Frith. Levels of emotional awareness and autism: an fmri study. Social neuroscience, 3(2):97-112, 2008.

[274] Manuel F. Casanova et al. Minicolumnar abnormalities in autism. Acta Neuropathol., 112(3):287-303, 2006.

[275] Mohd Ali Balafar, Abdul Rahman Ramli, M Iqbal Saripan, and Syamsiah Mashohor. Review of brain MRI image segmentation methods. Artificial Intelligence Review, 33(3):261-274, 2010. 
[276] Neil I Weisenfeld and Simon K Warfield. Automatic segmentation of newborn brain MRI. Neuroimage, 47(2):564-572, 2009.

[277] Andrea UJ Mewes, Petra S Hüppi, Heidelise Als, Frank J Rybicki, Terrie E Inder, Gloria B McAnulty, Robert V Mulkern, Richard L Robertson, Michael J Rivkin, and Simon K Warfield. Regional brain development in serial magnetic resonance imaging of low-risk preterm infants. Pediatrics, 118(1):23-33, 2006.

[278] Hui Xue, Latha Srinivasan, Shuzhou Jiang, Mary Rutherford, A David Edwards, Daniel Rueckert, and Joseph V Hajnal. Automatic segmentation and reconstruction of the cortex from neonatal MRI. Neuroimage, 38(3):461-477, 2007.

[279] AJ Barkovich. Magnetic resonance techniques in the assessment of myelin and myelination. Journal of inherited metabolic disease, 28(3):311-343, 2005.

[280] Amit M Mathur, Jeffrey J Neil, Robert C McKinstry, and Terrie E Inder. Transport, monitoring, and successful brain MR imaging in unsedated neonates. Pediatric radiology, 38(3):260-264, 2008.

[281] HP Ng, SH Ong, KWC Foong, PS Goh, and WL Nowinski. Medical image segmentation using K-means clustering and improved watershed algorithm. In Image Analysis and Interpretation, 2006 IEEE Southwest Symposium on, pages 61-65. IEEE, 2006.

[282] Tao Song, Mo M Jamshidi, Roland R Lee, and Mingxiong Huang. A modified probabilistic neural network for partial volume segmentation in brain MR image. Neural Networks, IEEE Transactions on, 18(5):1424-1432, 2007.

[283] Arnaldo Mayer and Hayit Greenspan. An adaptive mean-shift framework for MRI brain segmentation. Medical Imaging, IEEE Transactions on, 28(8):1238-1250, 2009.

[284] Ruogu Fang, Yu-hsin Joyce Chen, Ramin Zabih, and Tsuhan Chen. Tree-metrics graph cuts for brain MRI segmentation with tree cutting. In Image Processing Workshop (WNYIPW), 2010 Western New York, pages 10-13. IEEE, 2010.

[285] A Ortiz, JM Górriz, J Ramirez, and D Salas-Gonzalez. MR brain image segmentation by growing hierarchical SOM and probability clustering. Electronics Letters, 47(10):585-586, 2011.

[286] Brian Patenaude, Stephen M Smith, David N Kennedy, and Mark Jenkinson. A bayesian model of shape and appearance for subcortical brain segmentation. Neuroimage, 56(3):907-922, 2011.

[287] Liang Li, Mei Xie, Jingjing Gao, and Xingming Yue. MRI brain segmentation based on a three-dimensional markov random field model. In Unifying Electrical Engineering and Electronics Engineering, pages 1233-1239. Springer, 2014.

[288] J Bethanney Janney, A Aarthi, and S Rajesh Kumar Reddy. An automatic MRI brain segmentation by using adaptive mean-shift clustering framework. In Proceedings of International Conference on Internet Computing and Information Communications, pages 111-119. Springer, 2014. 
[289] Joachim Weber, Christian Doenitz, Alexander Brawanski, and Christoph Palm. Data-parallel MRI brain segmentation in clinical use. In Bildverarbeitung für die Medizin 2015, pages 389-394. Springer, 2015.

[290] Q Mahmood, A Chodorowski, and M Persson. Automated MRI brain tissue segmentation based on mean shift and fuzzy c-means using a priori tissue probability maps. IRBM, 36(3):185-196, 2015.

[291] Petronella Anbeek, Koen L Vincken, Floris Groenendaal, Annemieke Koeman, Matthias JP Van Osch, and Jeroen Van der Grond. Probabilistic brain tissue segmentation in neonatal magnetic resonance imaging. Pediatric research, 63(2):158-163, 2008.

[292] Li Wang, Feng Shi, Gang Li, Weili Lin, John H Gilmore, and Dinggang Shen. Integration of Sparse Multi-modality Representation and Geometrical Constraint for Isointense Infant Brain Segmentation. In Medical Image Computing and ComputerAssisted Intervention-MICCAI 2013, pages 703-710. Springer, 2013.

[293] Li Wang, Yaozong Gao, Feng Shi, Gang Li, John H Gilmore, Weili Lin, and Dinggang Shen. Links: Learning-based multi-source integration framework for segmentation of infant brain images. NeuroImage, 108:160-172, 2015.

[294] M. Altaye, S. K. Holland, M. Wilke, and C. Gaser. Infant brain probability templates for MRI segmentation and normalization. Neuroimage, 43(4):721-730, 2008.

[295] Zhuang Song, Suyash P Awate, Daniel J Licht, and James C Gee. Clinical neonatal brain mri segmentation using adaptive nonparametric data models and intensitybased markov priors. In International Conference on Medical Image Computing and Computer-Assisted Intervention, pages 883-890. Springer, 2007.

[296] Zhuang Song, Nicholas Tustison, Brian Avants, and James Gee. Adaptive graph cuts with tissue priors for brain MRI segmentation. In Biomedical Imaging: Nano to Macro, 2006. 3rd IEEE International Symposium on, pages 762-765. IEEE, 2006.

[297] Wenlu Zhang, Rongjian Li, Houtao Deng, Li Wang, Weili Lin, Shuiwang Ji, and Dinggang Shen. Deep convolutional neural networks for multi-modality isointense infant brain image segmentation. NeuroImage, 108:214-224, 2015.

[298] Pim Moeskops, Manon JNL Benders, Sabina M Chit, Karina J Kersbergen, Floris Groenendaal, Linda S de Vries, Max A Viergever, and Ivana Išgum. Automatic segmentation of MR brain images of preterm infants using supervised classification. NeuroImage, 118:628-641, 2015.

[299] John Ashburner and Karl J Friston. Unified segmentation. Neuroimage, 26(3):839_ 851, 2005.

[300] Kilian M Pohl, John Fisher, W Eric L Grimson, Ron Kikinis, and William M Wells. A bayesian model for joint segmentation and registration. NeuroImage, 31(1):228239, 2006.

[301] Xiao Han and Bruce Fischl. Atlas renormalization for improved brain MR image segmentation across scanner platforms. Medical Imaging, IEEE Transactions on, 26(4):479-486, 2007. 
[302] Xabier Artaechevarria, Arrate Munoz-Barrutia, and Carlos Ortiz-de Solorzano. Combination strategies in multi-atlas image segmentation: Application to brain MR data. Medical Imaging, IEEE Transactions on, 28(8):1266-1277, 2009.

[303] Mert R Sabuncu, BT Thomas Yeo, Koen Van Leemput, Bruce Fischl, and Polina Golland. A generative model for image segmentation based on label fusion. Medical Imaging, IEEE Transactions on, 29(10):1714-1729, 2010.

[304] J-P Morin, Christian Desrosiers, and Luc Duong. Atlas-based segmentation of brain magnetic resonance imaging using random walks. In Computer Vision and Pattern Recognition Workshops (CVPRW), 2012 IEEE Computer Society Conference on, pages 44-49. IEEE, 2012.

[305] Jean-Michel Morel and Guoshen Yu. ASIFT: A new framework for fully affine invariant image comparison. SIAM Journal on Imaging Sciences, 2(2):438-469, 2009.

[306] Herbert Bay, Tinne Tuytelaars, and Luc Van Gool. Surf: Speeded up robust features. In Computer Vision-ECCV 2006, pages 404-417. Springer, 2006.

[307] Jyrki MP Lötjönen, Robin Wolz, Juha R Koikkalainen, Lennart Thurfjell, Gunhild Waldemar, Hilkka Soininen, and Daniel Rueckert. Fast and robust multi-atlas segmentation of brain magnetic resonance images. NeuroImage, 49(3):2352-2365, 2010.

[308] Fedde van der Lijn, Marleen de Bruijne, Stefan Klein, Tom den Heijer, Yoo Y Hoogendam, Aad van der Lugt, Monique MB Breteler, and Wiro J Niessen. Automated brain structure segmentation based on atlas registration and appearance models. Medical Imaging, IEEE Transactions on, 31(2):276-286, 2012.

[309] Yuri Boykov, Olga Veksler, and Ramin Zabih. Fast approximate energy minimization via graph cuts. Pattern Analysis and Machine Intelligence, IEEE Transactions on, 23(11):1222-1239, 2001.

[310] Christian Ledig, Robin Wolz, Paul Aljabar, J Lotjonen, Rolf A Heckemann, Alexander Hammers, and Daniel Rueckert. Multi-class brain segmentation using atlas propagation and EM-based refinement. In Biomedical Imaging (ISBI), 2012 9th IEEE International Symposium on, pages 896-899. IEEE, 2012.

[311] Vedran Srhoj-Egekher, MJNL Benders, Karina J Kersbergen, Max A Viergever, and I Isgum. Automatic segmentation of neonatal brain MRI using atlas based segmentation and machine learning approach. MICCAI Grand Challenge: Neonatal Brain Segmentation, 2012, 2012.

[312] Li Wang et al. Longitudinally guided level sets for consistent tissue segmentation of neonates. Human brain mapping, 34(4):956-972, 2013.

[313] F. Shi, Y. Fan, S. Tang, J. H. Gilmore, W. Lin, and D. Shen. Neonatal brain image segmentation in longitudinal MRI studies. Neuroimage, 49(1):391-400, 2010.

[314] Marie Cherel, Francois Budin, Marcel Prastawa, Guido Gerig, Kevin Lee, Claudia Buss, Amanda Lyall, Kirsten Zaldarriaga Consing, and Martin Styner. Automatic tissue segmentation of neonate brain MR images with subject-specific atlases. In 
SPIE Medical Imaging, pages 941311-941311. International Society for Optics and Photonics, 2015.

[315] Elsa D Angelini, Ting Song, Brett D Mensh, and Andrew F Laine. Segmentation and quantitative evaluation of brain MRI data with a multi-phase three-dimensional implicit deformable model. Medical imaging 2004: Image processing: 16-19 February 2004, San Diego, California, USA; Proceedings of SPIE, vol. 5370, 2004.

[316] Olivier Colliot, Oscar Camara, and Isabelle Bloch. Integration of fuzzy spatial relations in deformable models: Application to brain MRI segmentation. Pattern recognition, 39(8):1401-1414, 2006.

[317] Sanae Miri, Nicolas Passat, and Jean-Paul Armspach. Topology-preserving discrete deformable model: Application to multi-segmentation of brain MRI. In Image and Signal Processing, pages 67-75. Springer, 2008.

[318] Jia-Xiu Liu, Yong-Sheng Chen, and Li-Fen Chen. Accurate and robust extraction of brain regions using a deformable model based on radial basis functions. Journal of neuroscience methods, 183(2):255-266, 2009.

[319] A Albert Huang, Rafeef Abugharbieh, and Roger Tam. A Hybrid GeometricStatistical Deformable Model for Automated 3-D Segmentation in Brain MRI. Biomedical Engineering, IEEE Transactions on, 56(7):1838-1848, 2009.

[320] M Del Fresno, M Vénere, and Alejandro Clausse. A combined region growing and deformable model method for extraction of closed surfaces in 3D CT and MRI scans. Computerized Medical Imaging and Graphics, 33(5):369-376, 2009.

[321] Li Wang, Yunjie Chen, Xiaohua Pan, Xunning Hong, and Deshen Xia. Level set segmentation of brain magnetic resonance images based on local Gaussian distribution fitting energy. Journal of neuroscience methods, 188(2):316-325, 2010.

[322] Sami Bourouis and Kamel Hamrouni. 3D segmentation of MRI brain using level set and unsupervised classification. International Journal of Image and Graphics, 10(01):135-154, 2010.

[323] Cybèle Ciofolo and Christian Barillot. Atlas-based segmentation of 3D cerebral structures with competitive level sets and fuzzy control. Medical Image Analysis, 13(3):456-470, 2009.

[324] Xiao Hong Wang, Bin Liu, and Zhi Qiang Song. 3-dimensional brain MRI segmentation based on multi-layer background subtraction and seed region growing algorithm. In Applied Mechanics and Materials, volume 536, pages 218-221. Trans Tech Publ, 2014.

[325] Ming Zhao, Hsiao-Yu Lin, Chih-Hung Yang, Chih-Yu Hsu, Jeng-Shyang Pan, and Meng-Ju Lin. Automatic threshold level set model applied on MRI image segmentation of brain tissue. Appl. Math, 9(4):1971-1980, 2015.

[326] Amir Alansary, Marwa Ismail, Ahmed Soliman, Fahmi Khalifa, Matthew Nitzken, Ahmed Elnakib, Mahmoud Mostapha, Austin Black, Katie Stinebruner, Manuel F Casanova, et al. Infant brain extraction in t1-weighted mr images using bet and refinement using lcdg and mgrf models. IEEE journal of biomedical and health informatics, 20(3):925-935, 2016. 
[327] Amir Alansary, Ahmed Soliman, Matthew Nitzken, Fahmi Khalifa, Ahmed Elnakib, Mahmoud Mostapha, Manuel F Casanova, and Ayman El-Baz. An integrated geometrical and stochastic approach for accurate infant brain extraction. In 2014 IEEE International Conference on Image Processing (ICIP), pages 3542-3546. IEEE, 2014.

[328] P. A. Viola and W. M. Wells III. Alignment by maximization of mutual information. International Journal on Computer Vision, 24(2):137-154, 1997.

[329] Marwa Ismail, Ahmed Soliman, Ahmed ElTanboly, Andy Switala, Mona Mahmoud, Fahmi Khalifa, Georgy Gimel'farb, Manuel Casanova, Robert Keynton, and Ayman El-Baz. Detection of white matter abnormalities in $\mathrm{mr}$ brain images for diagnosis of autism in children. In Biomedical Imaging: From Nano to Macro, 2011 IEEE International Symposium on. IEEE, 2016.

[330] M Ismail, M Mostapha, A Soliman, M Nitzken, F Khalifa, A Elnakib, G Gimel'farb, MF Casanova, and A El-Baz. Segmentation of infant brain mr images based on adaptive shape prior and higher-order mgrf. In Image Processing (ICIP), 2015 IEEE International Conference on, pages 4327-4331. IEEE, 2015.

[331] A Alansary, A Soliman, F Khalifa, A Elnakib, M Mostapha, M Nitzken, M Casanova, and A El-Baz. Map-based framework for segmentation of mr brain images based on visual appearance and prior shape. MIDAS J, 1:1, 2013.

[332] Ayman El-Baz, Ahmed Elnakib, Fahmi Khalifa, Mohamed Abou El-Ghar, Patrick McClure, Ahmed Soliman, and Georgy Gimelrfarb. Precise segmentation of 3-d magnetic resonance angiography. IEEE Transactions on Biomedical Engineering, 59(7):2019-2029, 2012.

[333] A. Farag, A. El-Baz, and G. Gimel'farb. Precise segmentation of multimodal images. IEEE Transaction on Image Processssing, 15(4):952-968, 2006.

[334] Julian Besag. On the statistical analysis of dirty pictures. J. Royal Stat. Soc. Series $B, 48(3): 259-302,1986$.

[335] (ABIDE). http://fcon_1000.projects.nitrc.org/indi/abide/.

[336] Dan Hall, Michael F Huerta, Matthew J McAuliffe, and Gregory K Farber. Sharing heterogeneous data: the national database for autism research. Neuroinformatics, 10(4):331-339, 2012.

[337] Dice LR. Measures of the amount of ecologic association between species. Ecology, 26:297-302, 1945.

[338] Guido Gerig, Matthieu Jomier, and Miranda Chakos. Valmet: A new validation tool for assessing and improving $3 \mathrm{~d}$ object segmentation. In International Conference on Medical Image Computing and Computer-Assisted Intervention, pages 516-523. Springer, 2001.

[339] Yakang Dai, Feng Shi, Li Wang, Guorong Wu, and Dinggang Shen. ibeat: a toolbox for infant brain magnetic resonance image processing. Neuroinformatics, 11(2):211225, 2013.

[340] Rahul S Desikan, Florent Ségonne, Bruce Fischl, Brian T Quinn, Bradford C Dickerson, Deborah Blacker, Randy L Buckner, Anders M Dale, R Paul Maguire, 
Bradley T Hyman, et al. An automated labeling system for subdividing the human cerebral cortex on mri scans into gyral based regions of interest. Neuroimage, 31(3):968-980, 2006.

[341] Guido Gerig, Martin Styner, D Jones, Daniel Weinberger, and Jeffrey Lieberman. Shape analysis of brain ventricles using spharm. In Mathematical Methods in Biomedical Image Analysis, 2001. MMBIA 2001. IEEE Workshop on, pages 171178. IEEE, 2001.

[342] Moo K Chung, Kim M Dalton, and Richard J Davidson. Tensor-based cortical surface morphometry via weighted spherical harmonic representation. IEEE transactions on medical imaging, 27(8):1143-1151, 2008.

[343] The CGAL Project. CGAL User and Reference Manual. CGAL Editorial Board, 4.7 edition, 2015.

[344] D. Adalsteinsson. A fast level set method for propagating interfaces. J. Comput. Phys., 118(2):269-277, 1995.

[345] Aly A Farag, Ayman S El-Baz, and Georgy Gimel'farb. Precise segmentation of multimodal images. IEEE Transactions on Image Processing, 15(4):952-968, 2006.

[346] Scott-Van Zeeland, A Ashley, Mirella Dapretto, Dara G Ghahremani, Russell A Poldrack, and Susan Y Bookheimer. Reward processing in autism. Autism Research, 3(2):53-67, 2010.

[347] Jack L Lancaster, Diana Tordesillas-Gutiérrez, Michael Martinez, Felipe Salinas, Alan Evans, Karl Zilles, John C Mazziotta, and Peter T Fox. Bias between mni and talairach coordinates analyzed using the icbm- 152 brain template. Human brain mapping, 28(11):1194-1205, 2007.

[348] Simon B Eickhoff, Klaas E Stephan, Hartmut Mohlberg, Christian Grefkes, Gereon R Fink, Katrin Amunts, and Karl Zilles. A new spm toolbox for combining probabilistic cytoarchitectonic maps and functional imaging data. Neuroimage, 25(4):1325-1335, 2005.

[349] Yoshua Bengio, Pascal Lamblin, Dan Popovici, Hugo Larochelle, et al. Greedy layer-wise training of deep networks. Advances in neural information processing systems, 19:153, 2007.

[350] Ehsan Hosseini-Asl, Jacek M Zurada, and Olfa Nasraoui. Deep learning of partbased representation of data using sparse autoencoders with nonnegativity constraints. IEEE Transactions on Neural Networks and Learning Systems, 2015.

[351] Bruce Fischl. Freesurfer. Neuroimage, 62(2):774-781, 2012.

[352] National Kidney Foundation. About Chronic Kidney Disease [Online]. Available. https://www.kidney.org/kidneydisease/aboutckd.

[353] National Kidney Foundation. Organ Donation and Transplantion Statisitcs [Online]. Available. https://www.kidney.org/news/newsroom/factsheets/ Organ-Donation-and-Transplantation-Stats.

[354] D Rush and Winnipeg Transplant Group. Insights into subclinical rejection. In Transplantation proceedings, volume 36, pages S71-S73, 2004. 
[355] Seniha E. Yuksel, Ayman El-Baz, Aly A. Farag, M. E. Abo El-Ghar, Tarek A. Eldiasty, and Mohamed A. Ghoneim. Automatic detection of renal rejection after kidney transplantation. In International Congress Series, volume 1281, pages 773-778, 2005.

[356] A. Farag, A. El-Baz, S. Yuksel, M. Abou El-Ghar, and T. Eldiasty. A framework for the detection of acute rejection with Dynamic Contrast Enhanced Magnetic Resonance Imaging. In Proceedings of IEEE International Symposium on Biomedical Imaging: From Nano to Macro, (ISBI'06), pages 418-421, Arlington, Virginia, USA, April 6-9, 2006.

[357] A. El-Baz, A. Farag, R. Fahmi, S. Yuksel, M. Abo El-Ghar, and T. Eldiasty. Image analysis of renal DCE MRI for the detection of acute renal rejection. In Proceedings of IAPR International Conference on Pattern Recognition (ICPR'06), pages 822825, Hong Kong, August 20-24, 2006.

[358] A. El-Baz, A. Farag, R. Fahmi, S. Yuksel, W. Miller, M. Abou El-Ghar, T. El-Diasty, and M. Ghoneim. A new CAD system for the evaluation of kidney diseases using DCE-MRI. In Proceedings of International Conference on Medical Image Computing and Computer-Assisted Intervention, (MICCAI'08), pages 446-453, Copenhagen, Denmark, October 1-6, 2006.

[359] A. El-Baz, G. Gimel'farb, and M. Abo El-Ghar. Image analysis approach for identification of renal transplant rejection. In Proceedings of IAPR International Conference on Pattern Recognition, (ICPR'08), pages 1-4, Tampa, Florida, USA, December 8-11, 2008.

[360] Fahmi Khalifa, Ayman El-Baz, Georgy Gimel'farb, and Mohammed Abu El-Ghar. Non-invasive image-based approach for early detection of acute renal rejection. In Proc. Int. Conf. Med. Image Comput. Computer-Assisted Intervention (MICCAI'10), Beijing, China, Sept. 20-24, pages 10-18, 2010.

[361] F. Khalifa, G. M. Beache, M. Abou El-Ghar, T. El-Diasty, G. Gimel'farb, M. Kong, and A. El-Baz. Dynamic contrast-enhanced MRI-based early detection of acute renal transplant rejection. IEEE Transactions on Medical Imaging, 32(10):19101927, 2013.

[362] F. Khalifa, M. Abou El-Ghar, Behnaz Abdollahi, Hermann Frieboes, Tarek ElDiasty, and A. El-Baz. A comprehensive non-invasive framework for automated evaluation of acute renal transplant rejection using DCE-MRI. NMR in Biomedicine, 26(11):1460-1470, 2013.

[363] Harriet C Thoeny, Dominik Zumstein, Sonja Simon-Zoula, Ute Eisenberger, Frederik De Keyzer, Lucie Hofmann, Peter Vock, Chris Boesch, Felix J Frey, and Peter Vermathen. Functional evaluation of transplanted kidneys with diffusion-weighted and BOLD MR imaging: Initial experience 1. Radiology, 241(3):812-821, 2006.

[364] Ute Eisenberger, Harriet C Thoeny, Tobias Binser, Mathias Gugger, Felix J Frey, Chris Boesch, and Peter Vermathen. Evaluation of renal allograft function early after transplantation with diffusion-weighted MR imaging. European Radiology, 20(6):1374-1383, 2010. 
[365] S Palmucci, LA Mauro, P Veroux, G Failla, P Milone, GC Ettorre, N Sinagra, G Giuffrida, D Zerbo, and M Veroux. Magnetic resonance with diffusion-weighted imaging in the evaluation of transplanted kidneys: Preliminary findings. In Transplantation Proceedings, volume 43, pages 960-966, 2011.

[366] S Palmucci, LA Mauro, G Failla, PV Foti, P Milone, N Sinagra, D Zerbo, P Veroux, GC Ettorre, and M Veroux. Magnetic resonance with diffusion-weighted imaging in the evaluation of transplanted kidneys: Updating results in 35 patients. In Transplantation Proceedings, volume 44, pages 1884-1888, 2012.

[367] Peter Vermathen, Tobias Binser, Chris Boesch, Ute Eisenberger, and Harriet C Thoeny. Three-year follow-up of human transplanted kidneys by diffusion-weighted MRI and blood oxygenation level-dependent imaging. Journal of Magnetic Resonance Imaging, 35(5):1133-1138, 2012.

[368] Katarzyna Wypych-Klunder, Andrzej Adamowicz, Adam Lemanowicz, Wojciech Szczęsny, Zbigniew Włodarczyk, and Zbigniew Serafin. Diffusion-weighted MR imaging of transplanted kidneys: Preliminary report. Polish Journal of Radiology, 79:94-98, 2014.

[369] A Kaul, RK Sharma, RK Gupta, H Lal, A Yadav, D Bhadhuria, N Prasad, A Gupta, et al. Assessment of allograft function using diffusion-weighted magnetic resonance imaging in kidney transplant patients. Saudi Journal of Kidney Diseases and Transplantation, 25(6):1143, 2014.

[370] ME Abou-El-Ghar, TA El-Diasty, AM El-Assmy, HF Refaie, AF Refaie, and MA Ghoneim. Role of diffusion-weighted MRI in diagnosis of acute renal allograft dysfunction: A prospective preliminary study. The British Journal of Radiology, 85(1014):e206e211, 2014.

[371] Guangyi Liu, Fei Han, Wenbo Xiao, Qidong Wang, Ying Xu, and Jianghua Chen. Detection of renal allograft rejection using blood oxygen level-dependent and diffusion weighted magnetic resonance imaging: A retrospective study. BMC Nephrology, 15(1):158, 2014.

[372] Mohamed Shehata, Fahmi Khalifa, Ahmed Soliman, Ali Takieldeen, Mohamed Abou El-Ghar, Ahmed Shaffie, Amy C Dwyer, Rosemary Ouseph, Ayman El-Baz, and Keynton Robert. 3D diffusion MRI-based CAD system for early diagnosis of acute renal rejection. In Proceedings of IEEE 13th International Symposium on Biomedical Imaging, (ISBI'16), pages 1177-1180. IEEE, 2016.

[373] Mohamed Shehata, Fahmi Khalifa, Ahmed Soliman, Mohamed Abou El-Ghar, Amy Dwyer, Rosemary Ouseph, and Ayman El-Baz. Early assessment of acute renal rejection. In 12th Annual Scientific Meeting of American Society for Diagnostics and Interventional Nephrology (ASDIN), Phoenix, Arizona, USA, Feb 19-21, 2016.

[374] Mohamed Shehata, Fahmi Khalifa, Ahmed Soliman, Ali Taki Eldeen, Mohamed Abou El-Ghar, Tarek Eldiasty, Ayman El-Baz, and Robert Keynton. An appearanceguided deformable model for 4D kidney segmentation using diffusion MRI. In X. Jiang A. El-Baz and Taylor \& Francis J. Suri, Eds, editors, Biomedical Image Segmentation: Advances and Trends, page (In Press). CRC, 2016. 
[375] Mohamed Shehata, Fahmi Khalifa, Ahmed Soliman, Rahaf Alrefai, Mohamed Abou El-Ghar, Amy C Dwyer, Rosemary Ouseph, and Ayman El-Baz. A level set-based framework for 3D kidney segmentation from diffusion MR images. In Proceedings of IEEE 22nd International Conference on Image Processing, (ICIP'15), pages 4441-4445. IEEE, 2015.

[376] Mohamed Shehata, Fahmi Khalifa, Ahmed Soliman, Rahaf Alrefai, Mohamed Abou El-Ghar, Amy C Dwyer, Rosemary Ouseph, and Ayman El-Baz. A novel framework for automatic segmentation of kidney from DW-MRI. In Proceedings of IEEE 12th International Symposium on Biomedical Imaging, (ISBI'15), pages 951-954. IEEE, 2015.

[377] M Shehata, F Khalifa, A Soliman, M Abou El-Ghar, A Dwyer, G Gimelfarb, R Keynton, and A El-Baz. A promising non-invasive cad system for kidney function assessment. In International Conference on Medical Image Computing and Computer-Assisted Intervention, pages 613-621. Springer, 2016.

[378] Fahmi Khalifa, Ahmed Soliman, Amy C Dwyer, Georgy Gimel'farb, and Ayman ElBaz. A random forest-based framework for $3 \mathrm{~d}$ kidney segmentation from dynamic contrast-enhanced ct images. In Image Processing (ICIP), 2016 IEEE International Conference on, pages 3399-3403. IEEE, 2016.

[379] F. Khalifa, A. Soliman, G. Gimel'farb, R. Ouseph, A. Dwyer, T. El-Diasty, and A. ElBaz. Models and methods for analyzing DCE-MRI: A review. Medical Physics, 41(12):1-32, 2014.

[380] Mahmoud Mostapha, Fahmi Khalifa, Amir Alansary, Ahmed Soliman, Georgy Gimel'farb, and Ayman El-Baz. Dynamic MRI-based computer aided diagnostic systems for early detection of kidney transplant rejection: A survey. In Proceedings of of Computational Models for Life Science, Sydney, Australia (CMLS), volume 1559, pages 297-306. AIP Publishing, 2013.

[381] Mohamed Abou El-Ghar, Aly Farag, Tarek El-Diasty, Ahmed Shokeir, Huda Refaie, Yasser Osman, Tarek Mohsen, and Mohamed Ghoneim. Computer aided detection of acute renal allograft dysfunction using dynamic contrast enhanced MRI. The Egyptian Journal of Radiology and Nuclear Medicine, 42(3):443-449, 2011.

[382] F. Khalifa, G. Beache, M. Nitzken, G. Gimel'farb, G. Giridharan, and A. El-Baz. Automatic analysis of left ventricle wall thickness using short-axis cine CMR images. In Proceedings of IEEE Int. Symp. Biomed. Imaging: From Nano to Macro, pages 1306-1309, Chicago, Illinois, USA, 30 March-2 April, 2011.

[383] Mahmoud Mostapha, Fahmi Khalifa, Amir Alansary, Ahmed Soliman, Jasjit Suri, and Ayman El-Baz. Computer-aided diagnosis systems for acute renal transplant rejection: Challenges and methodologies. In Abdomen and Thoracic Imaging, pages 1-35. Springer, 2014.

[384] Mohamed Shehata, Fahmi Khalifa, Elizabeth Hollis, Ahmed Soliman, Ehsan Hosseini-Asl, Mohamed Abou El-Ghar, Maryam El-Baz, Amy Dwyer, Ayman ElBaz, and Robert Keynton. A new non-invasive approach for early classification of renal rejection types using diffusion-weighted MRI. In Proceedings of IEEE 23rd 
International Conference on Image Processing, (ICIP'16), page (In Press). IEEE, 2016.

[385] Fahmi Khalifa, Ahmed Soliman, Ali Takieldeen, Mohamed Shehata, Mahmoud Mostapha, Ahmed Shaffie, Rosemary Ouseph, Adel Elmaghraby, and Ayman ElBaz. Kidney segmentation from CT images using a 3D NMF-guided active contour model. In Proceedings of IEEE 13th International Symposium on Biomedical Imaging, (ISBI'16), pages 432-435. IEEE, 2016.

[386] F. Khalifa, G. Beache, G. Gimel'farb, G. Giridharan, and A. El-Baz. Accurate automatic analysis of cardiac cine images. Transaction on Biomedical Engineering, 59(2):445-455, 2012.

[387] A. Firjani, A. Elmaghraby, and A. El-Baz. MRI-based diagnostic system for early detection of prostate cancer. In Biomedical Sciences and Engineering Conference (BSEC), 2013, pages 1-4, 2013.

[388] Ahmad Firjani, Ahmed Elnakib, Fahmi Khalifa, Ayman El-Baz, Georgy Gimel'farb, Mohamed Abou El-Ghar, and Adel Elmaghraby. A novel 3D segmentation approach for segmenting the prostate from dynamic contrast enhanced MRI using current appearance and learned shape prior. In Proceedings of IEEE International Symposium on Signal Processing and Information Technology, (ISSPIT'10), pages 137143, Luxor, Egypt, December 15-18, 2010.

[389] Ahmad Firjani, Ahmed Elnakib, Fahmi Khalifa, Georgy Gimelfarb, Mohamed Abou El-Ghar, Adel Elmaghraby, and Ayman El-Baz. A diffusion-weighted imaging based diagnostic system for early detection of prostate cancer. Journal of Biomedical Science and Engineering, 6(03):346, 2013.

[390] A. Firjani, A. Elnakib, F. Khalifa, G. Gimel'farb, M. Abou El-Ghar, J. Suri, A. Elmaghraby, and A. El-Baz. A new 3D automatic segmentation framework for accurate extraction of prostate from DCE-MRI. In Proceedings of IEEE International Symposium on Biomedical Imaging: From Nano to Macro, (ISBI'11), pages 1476-1479, Chicago, Illinois, March 30-April 2, 2011.

[391] A. Firjani, A. Elnakib, F. Khalifa, G. Gimelfarb, M. Abou El-Ghar, A. Elmaghraby, and A. El-Baz. A diffusion-weighted imaging based diagnostic system for early detection of prostate cancer. Journal of Biomedical Science and Engineering, 6(3A):346-356, 2013.

[392] A. Firjani, F. Khalifa, A. Elnakib, G. Gimel'farb, M. Abou El-Ghar, A. Elmaghraby, and A. El-Baz. 3D automatic approach for precise segmentation of the prostate from diffusion-weighted magnetic resonance imaging. In Proceedings of IEEE International Conference on Image Processing, (ICIP'11), pages 2285-2288, Brussels, Belgium, September 11-14, 2011.

[393] A. Firjani, F. Khalifa, A. Elnakib, G. Gimel'farb, M. Abou El-Ghar, A. Elmaghraby, and A. El-Baz. A novel image-based approach for early detection of prostate cancer using DCE-MRI. In Kenji Suzuki, editor, Computational Intelligence in Biomedical Imaging, pages 55-82. Springer New York, 2014.

[394] A. Firjani, F. Khalifa, A. Elnakib, G. Gimel'farb, A. Elmaghraby, and A. El-Baz. A novel image-based approach for early detection of prostate cancer. In Proceedings of 
IEEE International Conference on Image Processing, (ICIP'12), pages 2849-2852, Lake Buena Vista, Florida, September 30-October 3, 2012.

[395] Islam Reda, Ahmed Shalaby, Mohamed Abou El-Ghar, Fahmi Khalifa, Mohammed Elmogy, Ahmed Aboulfotouh, Ehsan Hosseini-Asl, Ayman El-Baz, and Robert Keynton. A new NMF-autoencoder based CAD system for early diagnosis of prostate cancer. In Proceedings of IEEE 13th International Symposium on Biomedical Imaging (ISBI'16), pages 1237-1240. IEEE, 2016.

[396] Ahmad Firjany, Ahmed Elnakib, Ayman El-Baz, Georgy Gimelfarb, Mohamed Abou El-Ghar, and Adel Elmagharby. Novel stochastic framework for accurate segemntation of prostate in dynamic contrast-enhanced MRI. In Proceedings of the International Wokshop on Prostate Cancer Imaging: Computer-Aided Diagnosis, Prognosis, and Intervention, pages 121-130, Beijing, China, Sptember 24, 2010.

[397] P. McClure, F. Khalifa, A Soliman, M Abou El-Ghar, G Gimelfarb, A Elmagraby, and A El-Baz. A novel nmf guided level-set for dwi prostate segmentation. Journal of Computer Science and Systems Biology, 7:209-216, 2014.

[398] A. Firjani, A. Elnakib, F. Khalifa, G. Gimel'farb, M. Abou El-Ghar, A. Elmaghraby, and A. El-Baz. A new 3D automatic segmentation framework for accurate extraction of prostate from diffusion imaging. In Proceedings of Biomedical Science and Engineering Conference-Image Informatics and Analytics in Biomedicine, (BSEC'11), pages 1306-1309, 2011.

[399] A. Firjani, F. Khalifa, A. Elnakib, G. Gimel'farb, M. Abou El-Ghar, A. Elmaghraby, and A. El-Baz. Non-invasive image-based approach for early detection of prostate cancer. In Proceedings of Fourth International Conference on Developments in eSystems Engineering, (DeSE'11), pages 172-177, Dubai, UAE, December 6-8, 2011.

[400] Ahmed Elnakib, Garth M Beache, Georgy Gimelfarb, and Ayman El-Baz. Intramyocardial strain estimation from cardiac cine mri. International journal of computer assisted radiology and surgery, 10(8):1299-1312, 2015.

[401] G. Beache, F. Khalifa, G. Gimel'farb, and A. El-Baz. Fully automated framework for the analysis of myocardial first-pass perfusion MR images. Medical Physics, 41(10):1-18, 2014.

[402] A. Elnakib, G. Beache, G. Gimel'farb, and A. El-Baz. New automated MarkovGibbs random field based framework for myocardial wall viability quantification on agent enhanced cardiac magnetic resonance images. The International Journal of Cardiovascular Imaging, 28(7):1683-1698, 2012.

[403] A. Elnakib, G. M. Beache, G. Gimel'farb, and A. El-Baz. A new framework for automated segmentation of left ventricle wall from contrast enhanced cardiac magnetic resonance images. In Proceeding of International Conference on Image Processing (ICIP'2011), pages 2289-2292. IEEE, 2011.

[404] A. Elnakib, G. Beache, G. Gimel'farb T. Inanc, and A. El-Baz. Validating a new methodology for strain estimation from cardiac cine MRI. In Proceedings of International Symposium on Computational Models for Life Science (CMLS'13), 2013. 
[405] A. Elnakib, G. Beache, M. Nitzken, G. Gimel'farb, and A. El-Baz. A new framework for automated identification of pathological tissues in contrast enhanced cardiac magnetic resonance images. In Proceedings of IEEE International Symposium on Biomedical Imaging: From Nano to Macro (ISBI'2011), pages 1272-1275. IEEE, 2011.

[406] A. Elnakib, G. Beache, H. Sliman, G. Gimel'farb, T. Inanc, and A. El-Baz. A novel Laplace-based method to estimate the strain from cine cardiac magnetic resonance images. In Proceedings of IEEE International Conference on Image Processing (ICIP'2013). IEEE, 2013.

[407] F. Khalifa, G. Beache, A. El-Baz, and G. Gimel'farb. Deformable model guided by stochastic speed with application in cine images segmentation. In Proceedings of IEEE International Conference on Image Processing, (ICIP'10), pages 1725-1728, Hong Kong, September 26-29, 2010.

[408] F. Khalifa, G. Beache, A. Elnakib, H. Sliman, G. Gimel'farb, K. C. Welch, and A. El-Baz. A new shape-based framework for the left ventricle wall segmentation from cardiac first-pass perfusion MRI. In Proceedings of IEEE International Symposium on Biomedical Imaging: From Nano to Macro, (ISBI'13), pages 41-44, San Francisco, CA, April 7-11, 2013.

[409] F. Khalifa, G. Beache, A. Elnakib, H. Sliman, G. Gimel'farb, K. Welch, and A. ElBaz. A new nonrigid registration framework for improved visualization of transmural perfusion gradients on cardiac first-pass perfusion MRI. In Proceedings of IEEE International Symposium on Biomedical Imaging: From Nano to Macro, (ISBI'12), pages 828-831, Barcelona, Spain, May 2-5, 2012.

[410] F. Khalifa, G. Beache, A. Firjani, K. Welch, G. Gimel'farb, and A. El-Baz. A new nonrigid registration approach for motion correction of cardiac first-pass perfusion MRI. In Proceedings of IEEE International Conference on Image Processing, (ICIP'12), pages 1665-1668, Lake Buena Vista, Florida, September 30-October 3, 2012.

[411] F. Khalifa, G. Beache, G. Gimel'farb, and A. El-Baz. A novel CAD system for analyzing cardiac first-pass MR images. In Proceedings of IAPR International Conference on Pattern Recognition (ICPR'12), pages 77-80, Tsukuba Science City, Japan, November 11-15, 2012.

[412] F. Khalifa, G. M. Beache, G. Gimel'farb, and A. El-Baz. A novel approach for accurate estimation of left ventricle global indexes from short-axis cine MRI. In Proceedings of IEEE International Conference on Image Processing, (ICIP'11), pages 2645-2649, Brussels, Belgium, September 11-14, 2011.

[413] F. Khalifa, G. Beache, G. Gimel'farb, G. Giridharan, and A. El-Baz. A new imagebased framework for analyzing cine images. In A. El-Baz, U. Acharya, M. Mirmedhdi, and J. Suri, editors, Handbook of Multi Modality State-of-the-Art Medical Image Segmentation and Registration Methodologies, volume 2, chapter 3, pages 69-98. Springer, New York, 2011. 
[414] F. Khalifa, G.Beache, G. Gimel'farb, G. Giridharan, and A. El-Baz. Accurate automatic analysis of cardiac cine images. IEEE Transactions on Biomedical Engineering, 59(2):445-455, 2012.

[415] M Nitzken, G Beache, A Elnakib, F Khalifa, G Gimel'farb, and A El-Baz. Accurate modeling of tagged $\mathrm{cmr} 3 \mathrm{D}$ image appearance characteristics to improve cardiac cycle strain estimation. In Proceedings of $19^{\text {th }}$ IEEE International Conference onImage Processing (ICIP'12), pages 521-524, Orlando, Florida, USA, September 2012. IEEE.

[416] M Nitzken, A El-Baz, and G Beache. Markov-gibbs random field model for improved full-cardiac cycle strain estimation from tagged CMR. Journal of Cardiovascular Magnetic Resonance, 14(1):1-2, 2012.

[417] H Sliman, A Elnakib, G Beache, A Elmaghraby, and A El-Baz. Assessment of myocardial function from cine cardiac MRI using a novel 4D tracking approach. Journal of Computer Science and System Biology, 7:169-173, 2014.

[418] H. Sliman, A. Elnakib, G. Beache, A. Soliman, F. Khalifa, G. Gimel'farb, A. Elmaghraby, and A. El-Baz. A novel 4D PDE-based approach for accurate assessment of myocardium function using cine cardiac magnetic resonance images. In Proceeding of IEEE International Conference on Image Processing (ICIP'14), pages 3537-3541, Paris, France, October 27-30, 2014.

[419] H. Sliman, F. Khalifa, A. Elnakib, A. Soliman, G. Beache, A. Elmaghraby, G. Gimel'farb, and A. El-Baz. Myocardial borders segmentation from cine MR images using bi-directional coupled parametric deformable models. Medical Physics, 40(9):1-13, 2013.

[420] H. Sliman, F. Khalifa, A. Elnakib, G. Beache, A. Elmaghraby, and A. El-Baz. A new segmentation-based tracking framework for extracting the left ventricle cavity from cine cardiac MRI. In Proceedings of IEEE International Conference on Image Processing, (ICIP'13), pages 685-689, Melbourne, Australia, September 15-18, 2013.

[421] H. Sliman, F. Khalifa, A. Elnakib, A. Soliman, G. Beache, G. Gimel'farb, A. Emam, A. Elmaghraby, and A. El-Baz. Accurate segmentation framework for the left ventricle wall from cardiac cine MRI. In Proceedings of International Symposium on Computational Models for Life Science, (CMLS'13), volume 1559, pages 287-296, Sydney, Australia, November 27-29, 2013.

[422] Shlomit Schaal, Marwa Ismail, Agustina C Palacio, Ahmed ElTanboly, Andy Switala, Ahmed Soliman, Thomas Neyer, Amir Hajrasouliha, Amir Hadayer, Douglas Kenneth Sigford, et al. Subtle early changes in diabetic retinas revealed by a novel method that automatically quantifies spectral domain optical coherence tomography (sd-oct) images. Investigative Ophthalmology \& Visual Science, 57(12):6324-6324, 2016.

[423] Thomas Neyer, Ahmed ElTanboly, Agustina C Palacio, Marwa Ismail, Andy Switala, Ahmed Soliman, Amir Hajrasouliha, Amir Hadayer, Douglas Kenneth Sigford, Ayman El-Baz, et al. A novel automated method for the objective quantification of retinal layers based on spectral domain optical coherence tomography (sd- 
oct) imaging reveals sequential changes in the normal retina with age. Investigative Ophthalmology \& Visual Science, 57(12):5943-5943, 2016.

[424] A El Tanboly, M Ismail, A Switala, M Mahmoud, A Soliman, T Neyer, A Palacio, A Hadayer, M El-Azab, S Schaal, et al. A novel automated method for the objective quantication of retinal layers reveals sequential changes that occur in the normal retina with age. In Image Processing (ICIP), 2016 IEEE International Conference on, pages 116-120. IEEE, 2016.

[425] S Schaal, A El Tanboly, M Ismail, A Switala, A Shalaby, A Hadayer, O Abdelmegid, and A El-Baz. A novel automatic segmentation of healthy and diseased retinal layers from oct scans. In Frontiers in Nanoscience and Nanotechnology, 2016.

[426] Ahmed Soliman, Fahmi Khalifa, Neal Dunlap, Brian Wang, Mohamed Abou ElGhar, and Ayman El-Baz. An iso-surfaces based local deformation handling framework of lung tissues. In Proceedings of IEEE 13th International Symposium on Biomedical Imaging (ISBI'16), pages 1253-1259. IEEE, 2016.

[427] Ahmed Soliman, Fahmi Khalifa, Ahmed Shaffie, Neal Dunlap, Brian Wang, Adel Elmaghraby, and Ayman El-Baz. Detection of lung injury using 4D-CT chest images. In Proceedings of IEEE 13th International Symposium on Biomedical Imaging (ISBI'16), pages 1274-1277. IEEE, 2016.

[428] Ni Liu, Ahmed Soliman, Georgy Gimelfarb, and Ayman El-Baz. Segmenting kidney DCE-MRI using 1st-order shape and 5th-order appearance priors. In Proceedings of International Conference on Medical Image Computing and Computer-Assisted Intervention, (MICCAI'15), pages 77-84. Springer, 2015.

[429] Ayman El-Baz, Ahmed Elnakib, Mohamed Abou El-Ghar, Georgy Gimel'farb, Robert Falk, and Aly Farag. Automatic detection of 2D and 3D lung nodules in chest spiral CT scans. International Journal of Biomedical Imaging, 2013, 2013.

[430] Ayman El-Baz, Garth Beache, Georgy Gimel'farb, Kenji Suzuki, and Kazunori Okada. Lung imaging data analysis. International Journal of Biomedical Imaging, 2013, 2013.

[431] Ayman El-Baz, Garth Beache, Georgy Gimel'farb, Kenji Suzuki, Kazunori Okada, Ahmed Elnakib, Ahmed Soliman, and Behnoush Abdollahi. Computer-aided diagnosis systems for lung cancer: Challenges and methodologies. International Journal of Biomedical Imaging, 2013, 2013.

[432] B. Abdollahi, A. Civelek, X.-F. Li, J. Suri, and A. El-Baz. PET/CT nodule segmentation and diagnosis: A survey. In Luca Saba and Jasjit Suri, editors, Multi Detector CT Imaging, chapter 30, pages 639-651. Taylor , Francis, 2014.

[433] Ahmed Soliman, Fahmi Khalifa, Ahmed Elnakib, Mohamed Abou El-Ghar, Neal Dunlap, Brian Wang, Georgy Gimel'farb, Robert Keynton, and Ayman El-Baz. Accurate lungs segmentation on ct chest images by adaptive appearance-guided shape modeling. IEEE Transactions on Medical Imaging, 2016.

[434] Ahmed Elnakib, Manuel F Casanova, Ahmed Soliman, Georgy Gimelfarb, and Ayman El-Baz. Analysis of 3d corpus callosum images in the brains of autistic individ- 
uals. Handbook of Research on Trends in the Diagnosis and Treatment of Chronic Conditions, page 159, 2015.

[435] Marwa MT Ismail, Robert S Keynton, Mahmoud MMO Mostapha, Ahmed H ElTanboly, Manuel F Casanova, Georgy L Gimel'farb, and Ayman El-Baz. Studying autism spectrum disorder with structural and diffusion magnetic resonance imaging: A survey. Frontiers in Human Neuroscience, 10:211, 2016.

[436] M Mostapha, M Casanova, G Gimelfarb, and A El-Baz. Towards non-invasive image-based early diagnosis of autism. In Proceedings of International Conference on Medical Image Computing and Computer-Assisted Intervention (MICCAI'15), pages 160-168. Springer, 2015.

[437] Brynn Dombroski, Andrew Switala, Ayman El-Baz, and Manuel Casanova. Gyral window mapping of typical cortical folding using MRI. Translational neuroscience, 2(2):142-147, 2011.

[438] B. Dombroski, M. Nitzken, A. Elnakib, F. Khalifa, A. El-Baz, and M. Casanova. Cortical surface complexity in a population-based normative sample. Translational Neuroscience, 5(1):17-24, 2014.

[439] A. El-Baz, M. Casanova, G. Gimel'farb, M. Mott, and A. Switala. An MRI-based diagnostic framework for early diagnosis of dyslexia. International Journal of Computer Assisted Radiology and Surgery, 3(3-4):181-189, 2008.

[440] A. El-Baz, M. Casanova, G. Gimel'farb, M. Mott, A. Switala, E. Vanbogaert, and R. McCracken. A new CAD system for early diagnosis of dyslexic brains. In Proceedings of International Conference on Image Processing (ICIP'2008), pages 1820-1823. IEEE, 2008.

[441] Ayman El-Baz, Manuel Casanova, Georgy Gimel'farb, Meghan Mott, and Andrew Switala. Autism diagnostics by 3D texture analysis of cerebral white matter gyrifications. In Proceedings of International Conference on Medical Image Computing and Computer-Assisted Intervention (MICCAI'2007), pages 882-890. Springer, 2007.

[442] Ayman El-Baz, Manuel Casanova, Georgy Gimel'farb, Meghan Mott, and Andrew Switwala. A new image analysis approach for automatic classification of autistic brains. In Proceedings of IEEE International Symposium on Biomedical Imaging: From Nano to Macro (ISBI'2007), pages 352-355. IEEE, 2007.

[443] A. El-Baz, A. Elnakib, F. Khalifa, M. Abou El-Ghar, P. McClure, A. Soliman, and G. Gimel'farb. Precise segmentation of 3-D magnetic resonance angiography. IEEE Transactions on Biomedical Engineering, 59(7):2019-2029, 2012.

[444] Ayman El-Baz, Aly Farag, Georgy Gimel'farb, Mohamed Abou El-Ghar, and Tarek Eldiasty. Probabilistic modeling of blood vessels for segmenting mra images. In ICPR (3), pages 917-920, 2006.

[445] Ayman El-Baz, Aly Farag, Georgy Gimelfarb, Mohamed Abou El-Ghar, and Tarek Eldiasty. A new adaptive probabilistic model of blood vessels for segmenting mra images. In Proceedings of Medical Image Computing and Computer-Assisted Intervention (MICCAI'06), volume 4191, pages 799-806. Springer, 2006. 
[446] Ayman El-Baz, Aly Farag, Georgy Gimelfarb, and Stephen Hushek. Automatic cerebrovascular segmentation by accurate probabilistic modeling of tof-mra images. In Medical Image Computing and Computer-Assisted Intervention (MICCAI'05), pages 34-42. Springer, 2005.

[447] A. El-Baz, A. Farag, A. Elnakib, M. Casanova, G. Gimel'farb, A. Switala, D. Jordan, and S. Rainey. Accurate automated detection of autism related corpus callosum abnormalities. Journal of Medical Systems, 35(5):929-939, 2011.

[448] A. El-Baz, Aymanand G. Gimel'farb M. Casanova G. Gimel'farb M. Mott El-Baz, M. Mott, A. Switala, E. Vanbogaert, and R. McCracken. Dyslexia diagnostics by 3D texture analysis of cerebral white matter gyrifications. In Proceedings of International Conference on Pattern Recognition (ICPR'2008), pages 1-4. IEEE, 2008.

[449] G. Gimel'farb-M. Mott A. El-Baz, M. Casanova and A. Switala. A new imagebased diagnostic framework for early diagnosis of dyslexic brains. In Proceedings of of Computer Assisted Radiology and Surgery (CARS'08), pages 46-47, 2008.

[450] A. Elnakib, A. El-Baz, M. Casanova, G. Gimel'farb, and A. Switala. Image-based detection of corpus callosum variability for more accurate discrimination between dyslexic and normal brains. In Proceedings of IEEE International Symposium on Biomedical Imaging: From Nano to Macro (ISBI'2010), pages 109-112. IEEE, 2010.

[451] A. Elnakib, A. El-Baz, M. F. Casanova, G. Gimel'farb, and A. Switala. Image-based detection of corpus callosum variability for more accurate discrimination between autistic and normal brains. In Proceedings of IEEE International Conference on Image Processing (ICIP'2010), pages 4337-4340. IEEE, 2010.

[452] A. Elnakib, A. El-Baz, M. Casanova, and A. Switala. Dyslexia diagnostics by centerline-based shape analysis of the corpus callosum. In Proceedings of International Conference on Pattern Recognition (ICPR'2010), pages 261-264. IEEE, 2010.

[453] A. Elnakib, M. Casanova, G. Gimel'farb, A. Switala, and A. El-Baz. Dyslexia diagnostics by 3-D shape analysis of the corpus callosum. IEEE Transactions on Information Technology in Biomedicine, 16(4):700-708, 2012.

[454] A. Elnakib, M. Casanova, G. Gimel'farb, A. Switala, and A. El-Baz. Autism diagnostics by centerline-based shape analysis of the corpus callosum. In Proceedings of IEEE International Symposium on Biomedical Imaging: From Nano to Macro (ISBI'2011), pages 1843-1846. IEEE, 2011.

[455] A. Elnakib, M. Casanova, G. Gimel'farb, and A. El-Baz. Autism diagnostics by 3D shape analysis of the corpus callosum. In K. Suzuki, editor, Machine Learning in Computer-aided Diagnosis: Medical Imaging Intelligence and Analysis, chapter 15, pages 315-335. IGI Global, Berlin, 2012.

[456] Rachid Fahmi, Ayman Elbaz, Hossam Hassan, Aly Farag, and Manuel Casanova. Structural MRI-based discrimination between autistic and typically developing brain. In Proceedings of Computer Assisted Radiology and Surgery (CARS'2007), pages 24-26, 2007. 
[457] Mahmoud Mostapha, Amir Alansary, Ahmed Soliman, Fahmi Khalifa, Matthew Nitzken, Rasha Khodeir, Manuel Casanova, and Ayman El-Baz. Atlas-based approach for the segmentation of infant DTI MR brain images. In Proceedings of IEEE $11^{\text {th }}$ International Symposium on Biomedical Imaging (ISBI'14), pages 1255-1258. IEEE, 2014.

[458] Mahmoud Mostapha, Ahmed Soliman, Fahmi Khalifa, Ahmed Elnakib, Amir Alansary, Matthew Nitzken, Manuel F Casanova, and Ayman El-Baz. A statistical framework for the classification of infant DT images. In Proceedings of IEEE International Conference Image Processing (ICIP'14), pages 2222-2226. IEEE, 2014.

[459] M Nitzken, M Casanova, G Gimel'farb, A Elnakib, F Khalifa, A Switala, and A ElBaz. 3D shape analysis of the brain cortex with application to dyslexia. In Proceedings of $18^{\text {th }}$ IEEE International Conference on Image Processing (ICIP)'11, pages 2657-2660, Brussels, Belgium, September 2011. IEEE.

[460] Matthew Nitzken et al. 3d shape analysis of the brain cortex with application to dyslexia. In Image Processing (ICIP), 2011 18th IEEE International Conference on, pages 2657-2660. IEEE, 2011.

[461] M Nitzken, M Casanova, F Khalifa, G Sokhadze, and A El-Baz. Shape-based detection of cortex variability for more accurate discrimination between autistic and normal brains. In A. El-Baz, R. Acharya, A. Laine, and J. Suri, editors, Handbook of Multi-Modality State-of-the-Art Medical Image Segmentation and Registration Methodologies, volume 2, chapter 7, pages 161-185. Springer Verlag, New York, March 2011.

[462] M Nitzken, M Casanova, and A El-Baz. Spharm analysis of the brain cortex for diagnosing dyslexia. In Proceedings of IEEE $11^{\text {th }}$ International Symposium on Biomedical Imaging (ISBI'14), Beijing, China, April 2014. IEEE.

[463] M Nitzken, G Casanova, Mand Gimelfarb, T Inanc, J Zurada, and A El-Baz. Shape analysis of the human brain: a brief survey. Journal of Biomedical and Health Informatics, 18(4):1337-1354, 2014.

[464] Ayman El-Baz, Georgy Gimelfarb, and Jasjit S Suri. Stochastic Modeling for Medical Image Analysis. CRC Press, 2015.

[465] Ayman El-Baz, Aly Farag, and Georgy Gimelfarb. Cerebrovascular segmentation by accurate probabilistic modeling of tof-mra images. In Scandinavian Conference on Image Analysis, pages 1128-1137. Springer, 2005.

[466] Ayman El-Baz, Georgy Gimelfarb, Ahmed Elnakib, Robert Falk, and Mohamed Abou El-Ghar. Fast, accurate unsupervised segmentation of 3d magnetic resonance angiography. In Atherosclerosis disease management, pages 411-432. Springer, 2011.

[467] Ayman El-Baz, Georgy Gimelfarb, Robert Falk, Mohamed Abou El-Ghar, Vedant Kumar, and David Heredia. A novel 3d joint markov-gibbs model for extracting blood vessels from pc-mra images. In International Conference on Medical Image Computing and Computer-Assisted Intervention, pages 943-950. Springer, 2009. 
[468] Ayman El-Baz, Georgy Gimel'farb, Vedant Kumar, Robert Falk, and Mohamed Abo El-Ghar. 3d joint markov-gibbs model for segmenting the blood vessels from mra. In 2009 IEEE International Symposium on Biomedical Imaging: From Nano to Macro, pages 1366-1369. IEEE, 2009. 


\section{CURRICULUM VITAE}

Name: Marwa Ismail

Contact Information

Department of Electrical and Computer Engineering

University of Louisville

Mobile: +1-502-821-6538

E-mail: mmarwa.ismail@gmail.com

Objective Pursuit of advanced research and development in image processing, and medical imaging as a postdoctoral fellow.

Research Interests My research/development interests lie in the fields of medical imaging and machine learning.

\section{Education}

University of Louisville, Louisville, Kentucky, USA

- Thesis Topic: A CAD system for early diagnosis of autism using different imaging modalities

- GPA : 3.82 out of 4

- Advisor: Professor Ayman El-Baz

\section{Refereed Journal Publications}

- Marwa Ismail, Robert Keynton, Mahmoud Mostapha, Ahmed ElTanboly, Manuel Casanova, Georgy Gimel?farb, and Ayman El-Baz..? Studying Autism Spectrum Disorder with Structural and Diffusion MRI: A Survey.? Frontiers in Human Neuroscience, 2016.

- Marwa Ismail, Amir Alansary*, Ahmed Soliman, Fahmi Khalifa, Matthew Nitzken, Ahmed Elnakib, Mahmoud Mostapha, Austin Black, Katie Stinebruner, Manuel F. Casanova, Jacek M. Zurada, and Ayman El-Baz.? Infant Brain Extraction in T1weighted MR Images using BET and Refinement using LCDG and MGRF Models.? IEEE Journal of Biomedical and Health Informatics, 2015.

- Marwa Ismail, Mohamed Sabry Hassouna, Salwa Elshazly, Aly Farag, Chuck Sites, Robert Curtin, Robert Falk, Albert Seow , and Gerald Dryden. ?Revamped Fly-Over for Accurate Colon Visualization in Virtual Colonoscopy.? IET journal, 2014. 
- Marwa Ismail, Ahmed ElTanboly*, Ahmed Shalaby*, Andy Switala, Shlomit Schaal, Georgy Gimel'farb, Magdi El?Azab, and Ayman El?Baz." A computer aided diagnostic system for detecting diabetic retinopathy in optical coherence tomography images." Medical physics journal, 2016.

\section{Conference Publications}

- Marwa Ismail, Ahmed ELTanboly*, A. Switala, M. Mahmoud, A. Soliman, T. Neyer, A. Palacio, A. Hadayer, M. El-Azab, S. Schaal, and A. El-Baz, ?A novel automatic segmentation of healthy and diseased retinal layers from OCT scans.? IEEE international conference In Image Processing (ICIP), pp. 116-120, September 2016.

- Marwa Ismail, Ahmed Soliman, Ahmed ELTanboly, Andy Switala, Mona Mahmoud, Fahmi Khalifa, Georgy Gimel'farb, Manuel Casanova, Robert Keynton, Ayman El-Baz. ?Detection of White Matter Abnormalities in MR Brain Images for Diagnosis of Autism in Children,? International Symposium on Biomedical Imaging, pp. 6-9, 2016.

- Marwa Ismail, Mahmoud Mostapha, Ahmed Soliman, Matthew Nitzken, Fahmi Khalifa, Ahmed Elnakib, Georgy Gimel'farb, Manuel Casanova, Ayman El-Baz. ?Segmentation of Infant MR Brain Images Based on Adaptive Shape Prior and HigherOrder MGRF,? IEEE international conference In Image Processing (ICIP), pp. 43274331, 2015.

- Marwa Ismail, Aly Farag, Salwa Elshazly, Robert Curtin, Robert Falk. "A Unified Framework for Automated Colon Segmentation.? In MICCAI 2014 Workshop on Abdominal Imaging: Computational and Clinical Applications, Boston, 2014

- Marwa Ismail, Shireen Elhabian, Aly Farag, and Gerald W. Dryden. ?Fully Automated 3D Colon Segmentation for Early Detection of Colorectal Cancer based on Convex Formulation of the Active Contour Model.? In CVPR Workshop on Medical Computer Vision, RI, 2012.

\section{Honors and Awards}

- Outstanding student award, graduate student 2015.

- Graduate Dean's Citation award 2016.

- Second place in E-Expo at the University of Louisville, 2011.

- Outstanding Teaching Assistant Award for Systems and Biomedical engineering Department, Cairo University, Egypt, 2008.

- An enlistee in Who's Who in America 2015.

- Reviewer in "Computational and Mathematical Methods in Medicine" journal. 
Skills Programming Languages and Toolkits

- $\mathrm{C}$ and $\mathrm{C}++$

- Matlab

- Java

- Visulization Toolkit (VTK)

- QT 